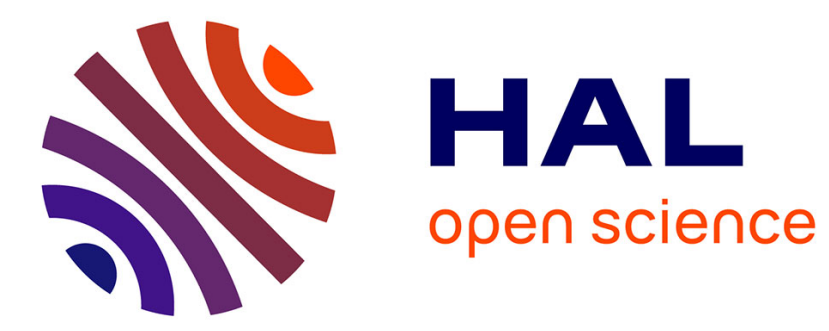

\title{
Espaces abstraits de morphismes et mutations
}

\author{
Jean-Marc Drézet
}

\section{To cite this version:}

Jean-Marc Drézet. Espaces abstraits de morphismes et mutations. Journal für die reine und angewandte Mathematik, 2000, 518, pp.41-93. hal-00742449v2

HAL Id: hal-00742449

https://hal.science/hal-00742449v2

Submitted on 19 Feb 2014

HAL is a multi-disciplinary open access archive for the deposit and dissemination of scientific research documents, whether they are published or not. The documents may come from teaching and research institutions in France or abroad, or from public or private research centers.
L'archive ouverte pluridisciplinaire HAL, est destinée au dépôt et à la diffusion de documents scientifiques de niveau recherche, publiés ou non, émanant des établissements d'enseignement et de recherche français ou étrangers, des laboratoires publics ou privés. 


\title{
ESPACES ABSTRAITS DE MORPHISMES ET MUTATIONS
}

\author{
JEAN-MARC DRÉZET
}

SOMMAIRE

1. Introduction 1

2. Un exemple simple 6

\begin{tabular}{lll}
\hline 3. & Mutations en termes de morphismes de faisceaux & 7 \\
\hline
\end{tabular}

4. Mutations abstraites 15

5. Le cas des morphismes de type $(r, s) \quad 33$

\begin{tabular}{lll}
6. & Applications & 46 \\
\hline
\end{tabular}

\begin{tabular}{ll}
\hline Références & 52
\end{tabular}

\section{INTRODUCTION}

\subsection{Variétés de modules de morphismes}

Soient $X$ une variété algébrique projective sur le corps des nombres complexes, et $\mathcal{E}, \mathcal{F}$ des faisceaux algébriques cohérents sur $X$. Soit

$$
W=\operatorname{Hom}(\mathcal{E}, \mathcal{F}) .
$$

Alors le groupe algébrique

$$
G=\operatorname{Aut}(\mathcal{E}) \times \operatorname{Aut}(\mathcal{F})
$$

agit d'une façon évidente sur $W$. Si deux morphismes sont dans la même $G$-orbite, leurs noyaux sont isomorphes, ainsi que leurs conoyaux. C'est pourquoi il peut être intéressant, pour décrire certaines variétés de modules de faisceaux, de construire des bons quotients d'ouverts $G$-invariants de $W$ par $G$. On s'intéresse au cas particulier suivant : soient $r, s$ des entiers positifs, $\mathcal{E}_{1}, \ldots, \mathcal{E}_{r}, \mathcal{F}_{1}, \ldots, \mathcal{F}_{s}$ des faisceaux cohérents sur $X$, qui sont simples, c'est-à-dire que leurs seuls endomorphismes sont les homothéties. On suppose aussi que

$$
\begin{gathered}
\operatorname{Hom}\left(\mathcal{E}_{i}, \mathcal{E}_{i^{\prime}}\right)=\{0\} \quad \text { si } i>i^{\prime}, \operatorname{Hom}\left(\mathcal{F}_{j}, \mathcal{F}_{j^{\prime}}\right)=\{0\} \quad \text { si } j>j^{\prime}, \\
\operatorname{Hom}\left(\mathcal{F}_{j}, \mathcal{E}_{i}\right)=\{0\} \text { pour tous } i, j .
\end{gathered}
$$

Soient $M_{1}, \ldots, M_{r}, N_{1}, \ldots, N_{s}$ des espaces vectoriels complexes de dimension finie. On suppose que

$$
\mathcal{E}=\bigoplus_{1 \leq i \leq r}\left(\mathcal{E}_{i} \otimes M_{i}\right), \mathcal{F}=\bigoplus_{1 \leq l \leq s}\left(\mathcal{F}_{l} \otimes N_{l}\right)
$$


Les éléments de $W$ sont appelés morphismes de type $(r, s)$. Le groupe $G$ n'est pas réductif en général. On a considéré dans [6] le problème de l'existence de bon quotients d'ouverts $G$ invariants de $\operatorname{Hom}(\mathcal{E}, \mathcal{F})$. On introduit une notion de semi-stabilité pour les morphismes de type $(r, s)$ qui dépend du choix d'une suite $\left(\lambda_{1}, \ldots, \lambda_{r}, \mu_{1}, \ldots, \mu_{s}\right)$ de nombres rationnels positifs tels que

$$
\sum_{1 \leq i \leq r} \lambda_{i} \operatorname{dim}\left(M_{i}\right)=\sum_{1 \leq l \leq s} \mu_{l} \operatorname{dim}\left(N_{l}\right)=1
$$

On appelle cette suite une polarisation de l'action de $G$. Il existe un bon quotient de l'ouvert des points semi-stables pour certaines valeurs de $\left(\lambda_{1}, \ldots, \lambda_{r}, \mu_{1}, \ldots, \mu_{s}\right)$ (ces résultats sont rappelés au $\S 5.4)$.

Les morphismes de type $(2,1)$ sont utilisés dans [2] pour décrire certaines variétés de modules de faisceaux semi-stables sur $\mathbb{P}_{2}$. Dans un certain nombre de travaux (cf. par exemple [10], [14]) des faisceaux semi-stables ou des faisceaux d'idéaux de sous-variétés de l'espace projectif sont décrits comme conoyaux de morphismes de type $(r, s)$.

Le but du présent article est de décrire et d'étudier certaines transformations, appelées mutations, associant à un morphisme de type $(r, s)$ un autre morphisme, pouvant être d'un autre type (mais la somme $r+s$ reste constante). On obtient en quelque sorte une correspondance entre deux espaces de morphismes $W$ et $W^{\prime}$, sur lesquels agissent respectivement les groupes en général non réductifs $G$ et $G^{\prime}$. Ceci permet de définir une bijection de l'ensemble des $G$-orbites d'un ouvert de $W$ sur l'ensemble des $G^{\prime}$-orbites d'un ouvert de $W^{\prime}$.

On associe de manière naturelle à chaque polarisation $\sigma$ de l'action de $G$ sur $W$ une polarisation $\sigma^{\prime}$ de l'action de $G^{\prime}$ sur $W^{\prime}$. Soit $W^{s s}$ (resp. $W^{\prime s s}$ ) l'ouvert de $W$ (resp. $W^{\prime}$ ) constitué des morphismes semi-stables relativement à $\sigma$ (resp. $\sigma^{\prime}$ ). Dans certains cas on montre qu'un point de $W$ est semi-stable relativement à $\sigma$ si et seulement si la mutation de ce point est semi-stable relativement à $\sigma^{\prime}$. On verra alors qu'il est possible (sous certaines conditions) de déduire de l'existence d'un bon quotient $W^{s s} / / G$ celle d'un bon quotient $W^{\prime s s} / / G^{\prime}$. C'est l'intérèt principal de l'étude des mutations de morphismes.

\subsection{Motivation}

La définition des mutations s'introduit naturellement lorsqu'on étudie les fibrés vectoriels algébriques sur $\mathbb{P}_{n}$ au moyen des suites spectrales de Beilinson généralisées (pour la définition, les propriétés et l'usage des suites spectrales de Beilinson généralisées sur les espaces projectifs, voir [1], [2], [3], [5],[7]). On associe une telle suite spectrale à un fibré $\mathcal{E}$ sur $\mathbb{P}_{n}$ et à une base d'hélice $\sigma$ de fibrés exceptionnels sur $\mathbb{P}_{n}$ (cf [1] et [7]). Si le diagramme de Beilinson correspondant à $\mathcal{E}$ est suffisamment simple, et sous certaines conditions, on obtient $\mathcal{E}$ comme conoyau d'un morphisme injectif du type

$$
\bigoplus_{1 \leq i \leq r}\left(E_{i} \otimes \mathbb{C}^{m_{i}}\right) \longrightarrow \bigoplus_{1 \leq l \leq s}\left(F_{l} \otimes \mathbb{C}^{n_{l}}\right)
$$

la base d'hélice $\sigma$ étant $\left(E_{1}, \ldots, E_{r}, F_{1}, \ldots, F_{s}\right)$ (et donc $r+s=n+1$ ). On peut, en changeant judicieusement la base d'hélice, obtenir d'autres représentations semblables de $\mathcal{E}$. On peut changer de base d'hélice en faisant subir à celle dont on part une série de transformations 
élémentaires appelées mutations, d'où la terminologie employée pour les transformations de morphismes étudiées ici.

Soient $W=\operatorname{Hom}\left(\bigoplus_{1 \leq i \leq r}\left(E_{i} \otimes \mathbb{C}^{m_{i}}\right), \bigoplus_{1 \leq l \leq s}\left(F_{l} \otimes \mathbb{C}^{n_{l}}\right)\right)$, et $w \in W$ le morphisme précédent. Soient $W_{i n j} \subset W$ l'ouvert constitué des morphismes injectifs, $\mathcal{U}$ le conoyau du morphisme universel évident sur $W_{i n j} \times \mathbb{P}_{n}$. On a donc $\mathcal{U}_{w} \simeq \mathcal{E}$. Il est facile de voir que le morphisme de déformation infinitésimale de Kodaïra-Spencer de $\mathcal{U}$ en $w$

$$
W \longrightarrow \operatorname{Ext}^{1}(\mathcal{E}, \mathcal{E})
$$

est surjectif, et que son noyau est l'espace tangent à l'orbite $G w$. Il est donc clair que les déformations de $\mathcal{E}$ sont en quelque sorte les $G$-orbites dans $W$ au voisinage de $G w$. On a une représentation analogue des déformations de $\mathcal{E}$ si on parvient à décrire celui-ci comme un conoyau d'un morphisme injectif en utilisant une autre base d'hélice : on obtient un autre espace de morphismes $W^{\prime}$ sur lequel agit un groupe $G^{\prime}$. Il y a donc une correspondance entre les $G$-orbites d'un ouvert de $W$ et les $G^{\prime}$-orbites d'un ouvert de $W^{\prime}$. On peut alors espérer décrire cette correspondance explicitement, et l'étendre formellement à des morphismes plus généraux.

On va décrire dans cet article une telle correspondance, qu'on pourrait appeler mutation non constructive. En effet, ce procédé a déjà été utilisé dans [4] : on définit plus généralement des mutations de complexes permettant de se ramener à des actions de groupes réductifs. On peut ainsi construire des quotients d'ouverts de $W$ par $G$ en travaillant sur l'espace sur lequel opère le groupe réductif, en employant la géométrie invariante classique. C'est pourquoi les mutations décrites dans [4] sont dites constructives. La construction des quotients d'espaces de morphismes de [6] en est un cas particulier.

\subsection{Mutations de morphismes}

\subsubsection{Définition succinte}

Soient $\mathcal{U}_{1}, \mathcal{U}_{2}, \mathcal{V}_{1}, \Gamma$ des faisceaux cohérents sur X. On suppose que $\Gamma$ est simple et que le morphisme canonique

$$
e v^{*}: \mathcal{U}_{2} \longrightarrow \Gamma \otimes \operatorname{Hom}\left(\mathcal{U}_{2}, \Gamma\right)^{*}
$$

est injectif. On note $\mathcal{V}_{2}$ son conoyau. On suppose que

$$
\operatorname{Hom}\left(\Gamma, \mathcal{U}_{2}\right)=\operatorname{Ext}^{1}\left(\Gamma, \mathcal{U}_{2}\right)=\{0\}
$$

On suppose de plus que

$$
\operatorname{Ext}^{1}\left(\mathcal{U}_{1}, \mathcal{U}_{2}\right)=\operatorname{Ext}^{1}\left(\Gamma, \mathcal{V}_{1}\right)=\operatorname{Ext}^{1}\left(\mathcal{U}_{1}, \Gamma\right)=\operatorname{Ext}^{1}\left(\mathcal{V}_{2}, \mathcal{V}_{1}\right)=\{0\}
$$

Soit $\Phi: \mathcal{U}_{1} \oplus \mathcal{U}_{2} \longrightarrow(\Gamma \otimes M) \oplus \mathcal{V}_{1}$ un morphisme injectif induisant une surjection $\lambda: \operatorname{Hom}\left(\mathcal{U}_{2}, \Gamma\right)^{*} \longrightarrow M$. On montre dans le théorème 3.2 qu'il existe un morphisme injectif

$$
\Phi^{\prime}: \mathcal{U}_{1} \oplus(\Gamma \otimes \operatorname{ker}(\lambda)) \longrightarrow \mathcal{V}_{2} \oplus \mathcal{V}_{1}
$$

tel que $\operatorname{coker}\left(\Phi^{\prime}\right) \simeq \operatorname{coker}(\Phi)$. On donne au $\S 3.2 .2$ une description précise du passage de $\Phi$ à $\Phi^{\prime}$. Cette description est à la base de l'étude abstraite faite ensuite. 
Dans le chapitre 4 on définit les mutations dans un cadre plus abstrait. On définit des actions de groupes sur des espaces vectoriels modelées sur les cas étudiés dans le chapitre précédent, c'està-dire qu'on formalise le passage de $\Phi$ à $\Phi^{\prime}$ étudié au chapitre 3 . Une mutation apparait alors comme une correspondance entre une telle action d'un groupe $G$ sur un espace vectoriel $V$ et une autre action d'un groupe $G^{\prime}$ sur un espace vectoriel $V^{\prime}$, de telle sorte qu'on ait une bijection $V^{0} / G \simeq V^{\prime 0} / G^{\prime}$, pour des ouverts adéquats $V^{0}$ et $V^{\prime 0}$ non vides de $V$ et $V^{\prime}$ respectivement. C'est un cas particulier de quasi-isomorphisme entre variétés sur lesquelles opèrent des groupes algébriques (cette notion introduite dans [4], ainsi que celle de quasi-isomorphisme fort sont rappelées au $\S 4.4 .2$ et des compléments sont apportés au $\S 4.4 .3$ dans le but de construire des bons quotients).

Soient $N$ un $\mathbb{C}$-espace vectoriel tel que $\operatorname{dim}(M)+\operatorname{dim}(N)=\operatorname{dim}\left(\operatorname{Hom}\left(\mathcal{U}_{2}, \Gamma\right)\right)$, et

$$
W=\operatorname{Hom}\left(\mathcal{U}_{1} \oplus \mathcal{U}_{2},(\Gamma \otimes M) \oplus \mathcal{V}_{1}\right), \quad W^{\prime}=\operatorname{Hom}\left(\mathcal{U}_{1} \oplus(\Gamma \otimes N), \mathcal{V}_{2} \oplus \mathcal{V}_{1}\right)
$$

Soient $W_{0}$ (resp. $W_{0}^{\prime}$ ) l'ouvert de $W$ (resp. $W^{\prime}$ ) constitué des morphismes induisant une surjection $\operatorname{Hom}\left(\mathcal{U}_{2}, \Gamma\right)^{*} \longrightarrow M$ (resp. une injection $\left.N \longrightarrow \operatorname{Hom}\left(\Gamma, \mathcal{V}_{2}\right)\right)$. Soient enfin

$$
G=\operatorname{Aut}\left(\mathcal{U}_{1} \oplus \mathcal{U}_{2}\right) \times \operatorname{Aut}\left((\Gamma \otimes M) \oplus \mathcal{V}_{1}\right), \quad G^{\prime}=\operatorname{Aut}\left(\mathcal{U}_{1} \oplus(\Gamma \otimes N)\right) \times \operatorname{Aut}\left(\mathcal{V}_{2} \oplus \mathcal{V}_{1}\right)
$$

Il résulte de l'étude abstraite du chapitre 4 que l'association de $\Phi^{\prime}$ à $\Phi$ induit une bijection $W_{0} / G \simeq W_{0}^{\prime} / G^{\prime}$. En fait on montre dans le théorème 4.11 qu'on a un quasi-isomorphisme fort, ce qui pour résumer signifie que cette bijection est fortement compatible avec l'action (algébrique) des groupes. La transformation réciproque (celle qui fait passer de $\Phi^{\prime}$ à $\Phi$ ) est formellement identique à la transformation directe (c'est encore plus clair si on suppose que tous les faisceaux cohérents qui interviennent sont localement libres). La mutation des morphismes est donc une transformation involutive.

\subsubsection{Le cas des morphismes de type $(r, s)$}

Dans le $\S 3.3$ on obtient le résultat suivant : soit

$$
\Phi: \bigoplus_{1 \leq i \leq r}\left(\mathcal{E}_{i} \otimes M_{i}\right) \longrightarrow \bigoplus_{1 \leq l \leq s}\left(\mathcal{F}_{l} \otimes N_{l}\right)
$$

un morphisme injectif, $\mathcal{U}$ son conoyau et $p$ un entier tel que $0 \leq p \leq r-1$. On suppose que pour $p+1 \leq j \leq r$ le morphisme canonique

$$
\mathcal{E}_{j} \longrightarrow \operatorname{Hom}\left(\mathcal{E}_{j}, \mathcal{F}_{1}\right)^{*} \otimes \mathcal{F}_{1}
$$

est injectif. Soit $\mathcal{G}_{j}$ son conoyau. Soit

$$
f_{p}: \bigoplus_{p+1 \leq j \leq r}\left(\operatorname{Hom}\left(\mathcal{E}_{j}, \mathcal{F}_{1}\right)^{*} \otimes M_{j}\right) \longrightarrow N_{1}
$$

l'application linéaire déduite de $\Phi$. On suppose que $f_{p}$ est surjective. Alors on montre que sous certaines hypothèses il existe une suite exacte

$$
0 \longrightarrow\left(\bigoplus_{1 \leq i \leq p}\left(\mathcal{E}_{i} \otimes M_{i}\right)\right) \oplus\left(\mathcal{F}_{1} \otimes \operatorname{ker}\left(f_{p}\right)\right) \longrightarrow\left(\bigoplus_{p<j \leq r}\left(\mathcal{G}_{j} \otimes M_{j}\right)\right) \oplus\left(\bigoplus_{2 \leq l \leq s}\left(\mathcal{F}_{l} \otimes N_{l}\right)\right) \longrightarrow \mathcal{U} \longrightarrow 0
$$


On a donc associé à un morphisme de type $(r, s)$ un morphisme de type $(p+1, r+s-p-1)$. Il suffit bien entendu d'appliquer les résultats du chapitre 3 avec

$$
\begin{gathered}
\mathcal{U}_{1}=\bigoplus_{1 \leq i \leq p}\left(\mathcal{E}_{i} \otimes M_{i}\right), \quad \mathcal{U}_{2}=\bigoplus_{p+1 \leq i \leq r}\left(\mathcal{E}_{i} \otimes M_{i}\right), \\
\Gamma=\mathcal{F}_{1}, \quad \mathcal{V}_{1}=\bigoplus_{2 \leq l \leq s}\left(\mathcal{F}_{l} \otimes N_{l}\right) .
\end{gathered}
$$

On associe naturellement au $\S 5.5$ à toute polarisation $\sigma$ de l'action de $G$ sur $W$ une polarisation $\sigma^{\prime}$ de l'action de $G^{\prime}$ sur $W^{\prime}$. Soit $W^{s s}$ (resp. $W^{\prime s s}$ ) l'ouvert de $W$ (resp. $W^{\prime}$ ) des points $G$-semistables relativement à $\sigma$ (resp. $G^{\prime}$-semi-stables relativement à $\sigma^{\prime}$ ). On montre que sous certaines conditions un point de $W_{0}$ est $G$-(semi-)stable relativement à $\sigma$ si et seulement si les points correspondants de $W_{0}^{\prime}$ sont $G^{\prime}$-(semi-)stables relativement à $\sigma^{\prime}$ (proposition 5.2). On en déduit dans les théorèmes 5.6, 5.7 et 5.8 que sous certaines conditions l'existence d'un bon quotient $W^{\prime s s} / / G^{\prime}$ découle de celle d'un bon quotient $W^{s s} / / G$.

Ceci permet d'étendre les résultats de [6] concernant les variétés de modules de morphismes de type $(2,1)$ (théorème 5.9). C'est-à-dire qu'on peut trouver d'autres polarisations telles qu'un bon quotient de l'ouvert des points semi-stables existe et est projectif.

\subsection{Plan des chapitres suivants}

Dans le chapitre 2 on donne un exemple simple et bien connu de mutations dans le cas des morphismes de type $(1,1)$. C'est ce type de résultats qu'il s'agit de généraliser.

Dans le chapitre 3 on donne la définition des mutations de morphismes en termes de faisceaux. On montre que si un faisceau cohérent peut être représenté comme conoyau d'un morphisme injectif de faisceaux, on peut dans certaines conditions le représenter aussi comme conoyau d'un morphisme injectif d'un autre type. On donne aussi une description précise des mutation de morphismes, qui sera formalisée ensuite.

Dans le chapitre 4 on décrit les espaces abstraits de morphismes qui sont des modélisations des espaces de morphismes étudiés au chapitre 3, avec l'action des groupes d'automorphismes. On décrit de manière abstraite la mutation d'un espace abstrait de morphismes, qui en est un autre. Les principaux résultats sont les théorèmes de quasi-isomorphisme 4.7 et 4.11 .

Dans le chapitre 5, on applique les résultats qui précèdent dans le but de trouver d'autres cas où on sait définir des bons quotients d'espaces de morphismes de type $(r, s)$. Dans le cas des morphismes de type $(2,1)$, le théorème 5.9 étend les résultats obtenus dans [6].

Dans le chapitre 6 on donne des exemples d'applications des résultats précédents. On s'intéresse ici aux morphisme du type

$$
\left(\mathcal{O}(-2) \otimes \mathbb{C}^{m_{1}}\right) \oplus\left(\mathcal{O}(-1) \otimes \mathbb{C}^{m_{2}}\right) \longrightarrow \mathcal{O} \otimes \mathbb{C}^{n_{1}}
$$

sur $\mathbb{P}_{n}$. La construction de bons quotients d'espaces de tels morphismes nécessite le calcul de certaines constantes dont on a donné des estimations dans [6]. Elles sont calculées exactement dans le $\S 6.1$. Ceci permet d'obtenir le théorème 6.4 qui donne les polarisations pour lesquelles 
on sait construire un bon quotient $W^{s s} / / G$ pour les morphismes du type précédent, aussi bien en utilisant directement les résultats de [6] que les mutations. On donne ensuite des exemples plus concrets. En particulier dans le $\S 6.4$ on étudie les morphismes

$$
\mathcal{O}(-2) \oplus\left(\mathcal{O}(-1) \otimes \mathbb{C}^{k}\right) \longrightarrow \mathcal{O} \otimes \mathbb{C}^{n k+1}
$$

sur $\mathbb{P}_{n}$. L'application directe de [6] ne donne que le bon quotient trivial, qui est un fibré en grassmanniennes sur la variété de modules des morphismes stables $\mathcal{O}(-1) \otimes \mathbb{C}^{k} \longrightarrow \mathcal{O} \otimes \mathbb{C}^{n k+1}$. En utilisant des mutations on peut construire d'autres quotients.

Remerciements. Je tiens à remercier G. Trautmann pour de nombreuses discussions qui m'ont beaucoup aidé, ainsi que l'Université de Kaiserslautern pour son hospitalité durant la réalisation d'une partie de ce travail. Je remercie aussi A. Rudakov pour ses suggestions (je lui dois en particulier le lemme 4.2).

\section{UN EXEMPLE SIMPLE}

Les résultats de ce chapitre sont démontrés dans [2]. Soient $L, M$ et $N$ des espaces vectoriels complexes de dimension finie, avec $\operatorname{dim}(L) \geq 3$. On pose $q=\operatorname{dim}(L), m=\operatorname{dim}(M)$, $n=\operatorname{dim}(N)$. Les applications linéaires

$$
L \otimes M \longrightarrow N
$$

sont appelées des L-modules de Kronecker. Soit

$$
W=\operatorname{Hom}(L \otimes M, N) .
$$

Sur $W$ opère de manière évidente le groupe algébrique réductif

$$
G=(G L(M) \times G L(N)) / \mathbb{C}^{*} .
$$

L'action de $S L(M) \times S L(N)$ sur $\mathbb{P}(W)$ se linéarisant de façon évidente, on a une notion de point (semi-) stable de $\mathbb{P}(W)$ (au sens de la géométrie invariante). On montre que si $f \in W, f$ est semi-stable (resp. stable) si et seulement si pour tous sous-espaces vectoriels $M^{\prime}$ de $M$ et $N^{\prime}$ de $N$, tels que $M^{\prime} \neq\{0\}, N^{\prime} \neq N$, et $f\left(L \otimes M^{\prime}\right) \subset N^{\prime}$, on a

$$
\frac{\operatorname{dim}\left(N^{\prime}\right)}{\operatorname{dim}\left(M^{\prime}\right)} \geq \frac{\operatorname{dim}(N)}{\operatorname{dim}(M)} \quad(\text { resp. }>\text { ). }
$$

Soit $W^{s s}$ (resp. $W^{s}$ ) l'ouvert des points semi-stables (resp. stables) de $W$. Alors il existe un bon quotient (resp. un quotient géométrique)

$$
N(L, M, N)=W^{s s} / / G \quad\left(\operatorname{resp} . \quad N_{s}(L, M, N)=W^{s} / G\right),
$$

$N(L, M, N)$ est projective, et $N_{s}(L, M, N)$ est un ouvert lisse de $N(L, M, N)$.

On pose $m^{\prime}=q m-n$. On suppose que $m^{\prime}>0$. Soit $M^{\prime}$ un espace vectoriel complexe de dimension $m^{\prime}$. Soit $f: L \otimes M \longrightarrow N$ un $L$-module de Kronecker surjectif. Alors 
$\operatorname{dim}(\operatorname{ker}(f))=m^{\prime}$. Soient

$$
f^{\prime}: L^{*} \otimes \operatorname{ker}(f) \longrightarrow M
$$

la restriction de l'application

$$
\operatorname{tr} \otimes I_{M}: L^{*} \otimes L \otimes M \longrightarrow M
$$

( $t r$ désignant l'application trace), et

$$
A(f): L^{*} \otimes M^{*} \longrightarrow \operatorname{ker}(f)^{*}
$$

l'application linéaire déduite de $f^{\prime}$, qu'on peut voir comme un élément de

$W^{\prime}=\operatorname{Hom}\left(L^{*} \otimes M^{*}, M^{\prime}\right)$, en utilisant un isomorphisme $\operatorname{ker}(f)^{*} \simeq M^{\prime}$. Soit $W_{0}$ l'ouvert de $W$ constitué des applications surjectives, $W_{0}^{\prime}$ l'ouvert analogue de $W^{\prime}$, et

$G^{\prime}=\left(G L\left(M^{*}\right) \times G L\left(M^{\prime}\right)\right) / \mathbb{C}^{*}$. On démontre aisément la

Proposition 2.1. 1 - En associant $A(f)$ à $f$ on définit une bijection

$$
W_{0} / G \simeq W_{0}^{\prime} / G^{\prime}
$$

2 - La bijection précédente induit un isomorphisme

$$
N(L, M, N) \simeq N\left(L^{*}, M^{*}, M^{\prime}\right)
$$

(induisant un isomorphisme $N_{s}(L, M, N) \simeq N_{s}\left(L^{*}, M^{*}, M^{\prime}\right)$ ).

On note plus simplement, si $q=\operatorname{dim}(L), m=\operatorname{dim}(M)$ et $n=\operatorname{dim}(N)$,

$$
N(q, m, n)=N(L, M, N), \quad N_{s}(q, m, n)=N_{s}(L, M, N) .
$$

\section{Mutations en termes De morphismes de FAisceaux}

On va d'abord démontrer un résultat général (prop. 3.1), qu'on appliquera ensuite dans le $\S 3.2$ à la définition des mutations de morphismes. Dans le $\S 3.1$ on étudie des faisceaux cohérents pouvant être représentés comme conoyaux de morphismes injectifs de faisceaux d'un certain type. Une étude similaire pourrait sans doute être faite sur les noyaux, ou dans un cadre encore plus général.

\subsection{Résultat général}

Soient $\mathcal{E}, \mathcal{E}^{\prime}, \mathcal{F}, \mathcal{F}^{\prime}$ et $\Gamma$ des faisceaux cohérents sur une variété projective irréductible $X$, avec $\Gamma$ simple. On suppose que le morphisme canonique

$$
e v: \Gamma \otimes \operatorname{Hom}(\Gamma, \mathcal{F}) \longrightarrow \mathcal{F}
$$

est surjectif. Soit $\mathcal{E}_{0}$ son noyau. On suppose que le morphisme canonique

$$
e v^{*}: \mathcal{E}^{\prime} \longrightarrow \Gamma \otimes \operatorname{Hom}\left(\mathcal{E}^{\prime}, \Gamma\right)^{*}
$$


est injectif. Soit $\mathcal{F}_{0}$ son conoyau. On suppose enfin que

$$
\operatorname{Hom}\left(\mathcal{E}^{\prime}, \mathcal{E}_{0}\right)=\operatorname{Ext}^{1}\left(\mathcal{E}^{\prime}, \mathcal{E}_{0}\right)=\operatorname{Ext}^{1}\left(\mathcal{F}_{0}, \mathcal{F}^{\prime}\right)=\operatorname{Ext}^{1}\left(\mathcal{E}, \mathcal{E}_{0}\right)=\{0\}
$$

De la suite exacte

$$
0 \longrightarrow \mathcal{E}_{0} \longrightarrow \Gamma \otimes \operatorname{Hom}(\Gamma, \mathcal{F}) \longrightarrow \mathcal{F} \longrightarrow 0
$$

on déduit un isomorphisme

$$
\operatorname{Hom}\left(\mathcal{E}^{\prime}, \mathcal{F}\right) \simeq \operatorname{Hom}\left(\operatorname{Hom}\left(\mathcal{E}^{\prime}, \Gamma\right)^{*}, \operatorname{Hom}(\Gamma, \mathcal{F})\right) .
$$

Si $\lambda \in \operatorname{Hom}\left(\operatorname{Hom}\left(\mathcal{E}^{\prime}, \Gamma\right)^{*}, \operatorname{Hom}(\Gamma, \mathcal{F})\right)$, le morphisme $\mathcal{E}^{\prime} \longrightarrow \mathcal{F}$ correspondant est la composée $\mathcal{E}^{\prime} \stackrel{e v^{*}}{\longrightarrow} \Gamma \otimes \operatorname{Hom}\left(\mathcal{E}^{\prime}, \Gamma\right)^{*} \stackrel{I_{\Gamma} \otimes \lambda}{\longrightarrow} \Gamma \otimes \operatorname{Hom}(\Gamma, \mathcal{F}) \stackrel{e v}{\longrightarrow} \mathcal{F}$.

Proposition 3.1. Soient

$$
\Phi: \mathcal{E} \oplus \mathcal{E}^{\prime} \longrightarrow \mathcal{F} \oplus \mathcal{F}^{\prime}
$$

un morphisme injectif de faisceaux et

$$
\lambda: \operatorname{Hom}\left(\mathcal{E}^{\prime}, \Gamma\right)^{*} \longrightarrow \operatorname{Hom}(\Gamma, \mathcal{F})
$$

l'application linéaire déduite du morphisme $\mathcal{E}^{\prime} \longrightarrow \mathcal{F}$ défini par $\Phi$.

1 - On suppose que $\lambda$ est surjective et que $\operatorname{Ext}^{1}(\mathcal{E}, \Gamma)=\{0\}$. Alors il existe un morphisme injectif

$$
\Phi^{\prime}: \mathcal{E} \oplus \mathcal{E}_{0} \oplus(\Gamma \otimes \operatorname{ker}(\lambda)) \longrightarrow \mathcal{F}_{0} \oplus \mathcal{F}^{\prime}
$$

tel que $\operatorname{coker}\left(\Phi^{\prime}\right) \simeq \operatorname{coker}(\Phi)$.

2 - On suppose que $\lambda$ est injective et que $\operatorname{Ext}^{1}\left(\Gamma, \mathcal{F}_{0}\right)=\operatorname{Ext}^{1}\left(\Gamma, \mathcal{F}^{\prime}\right)=\{0\}$. Alors il existe un morphisme injectif

$$
\Phi^{\prime \prime}: \mathcal{E} \oplus \mathcal{E}_{0} \longrightarrow(\Gamma \otimes \operatorname{coker}(\lambda)) \oplus \mathcal{F}_{0} \oplus \mathcal{F}^{\prime}
$$

tel que $\operatorname{coker}\left(\Phi^{\prime \prime}\right) \simeq \operatorname{coker}(\Phi)$.

Démonstration. On considère le morphisme $A: \mathcal{E}^{\prime} \longrightarrow\left(\Gamma \otimes \operatorname{Hom}\left(\mathcal{E}^{\prime}, \Gamma\right)^{*}\right) \oplus \mathcal{F}^{\prime}=\mathcal{A}$ dont la première composante est $e v^{*}$ et la seconde provient de $\Phi$. On a un diagramme commutatif avec lignes et colonnes exactes :

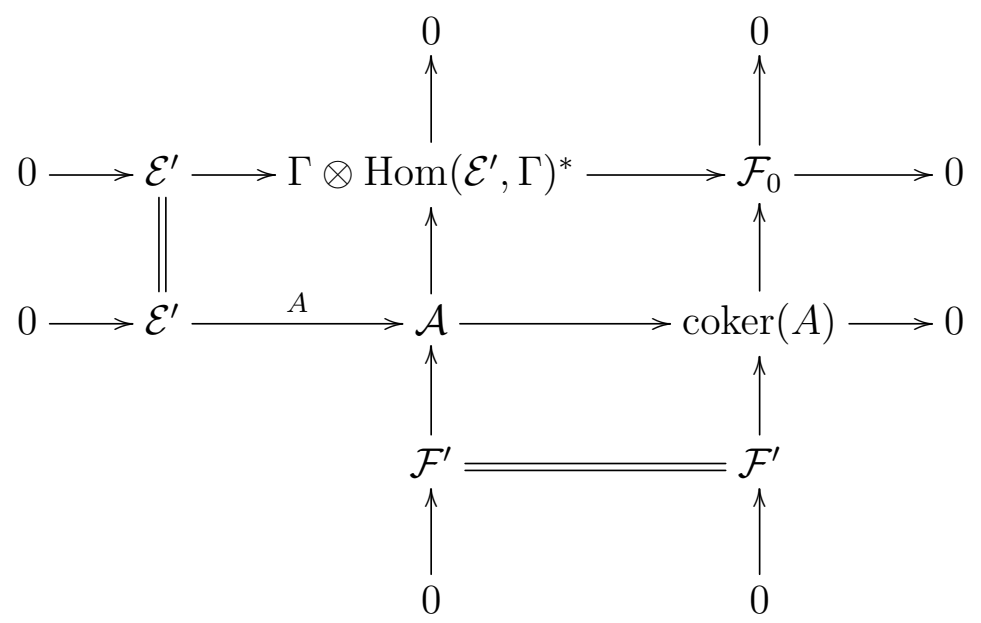


Puisque $\operatorname{Ext}^{1}\left(\mathcal{F}_{0}, \mathcal{F}^{\prime}\right)=\{0\}$, on a un isomorphisme $\operatorname{coker}(A) \simeq \mathcal{F}^{\prime} \oplus \mathcal{F}_{0}$.

On suppose maintenant que les hypothèses de 1- sont vérifiées. Soit

$$
\pi: \Gamma \otimes \operatorname{Hom}\left(\mathcal{E}^{\prime}, \Gamma\right)^{*} \longrightarrow \mathcal{F}
$$

le morphisme composé $\Gamma \otimes \operatorname{Hom}\left(\mathcal{E}^{\prime}, \Gamma\right)^{*} \stackrel{I_{\Gamma} \otimes \lambda}{\longrightarrow} \Gamma \otimes \operatorname{Hom}(\Gamma, \mathcal{F}) \stackrel{e v}{\longrightarrow} \mathcal{F}$. Alors on a

$$
\operatorname{ker}(\pi) \simeq \mathcal{E}_{0} \oplus(\operatorname{ker}(\lambda) \otimes \Gamma) .
$$

Soit $\mathcal{V}$ le conoyau du morphisme injectif $\mathcal{E}^{\prime} \longrightarrow \mathcal{F} \oplus \mathcal{F}^{\prime}$ déduit de $\Phi$. On a un diagramme commutatif avec lignes et colonnes exactes :

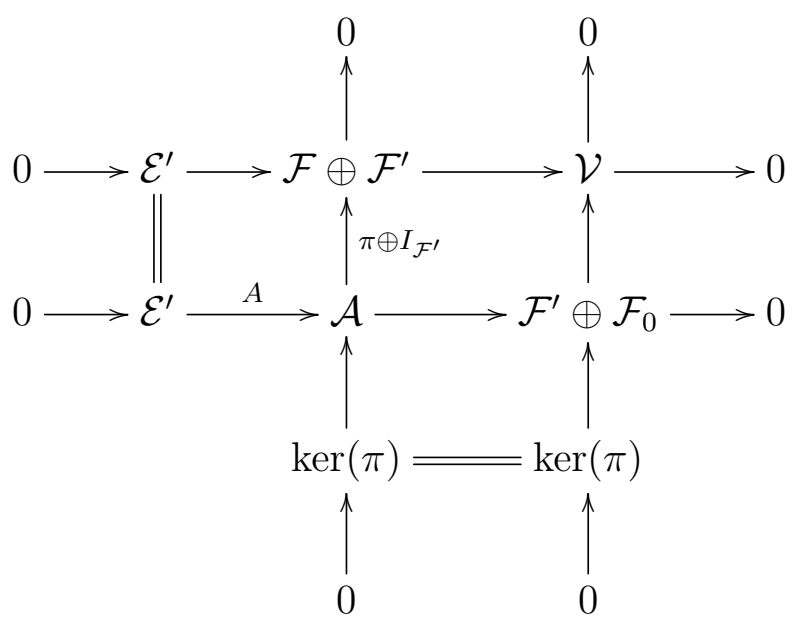

On a une suite exacte

$$
0 \longrightarrow \mathcal{E} \longrightarrow \mathcal{V} \longrightarrow \operatorname{coker}(\Phi) \longrightarrow 0
$$

et le morphisme $\mathcal{E} \longrightarrow \mathcal{V}$ se relève en un morphisme $\mathcal{E} \longrightarrow \mathcal{F}^{\prime} \oplus \mathcal{F}_{0} \quad\left(\operatorname{car}^{\operatorname{Ext}^{1}}\left(\mathcal{E}, \mathcal{E}_{0}\right)=\right.$ $\left.\operatorname{Ext}^{1}(\mathcal{E}, \Gamma)=\{0\}\right)$. On note $\mathcal{W}$ le conoyau de ce morphisme. On a alors un diagramme commutatif avec lignes et colonnes exactes, dont la ligne verticale du milieu provient du diagramme précédent :

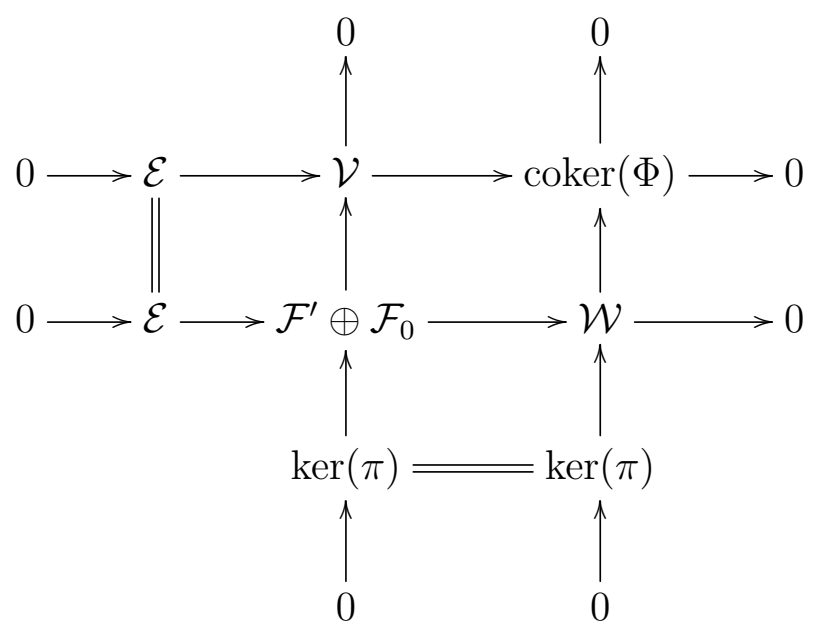


On en déduit une suite exacte

$$
0 \longrightarrow \mathcal{E} \oplus \operatorname{ker}(\pi) \longrightarrow \mathcal{F}^{\prime} \oplus \mathcal{F}_{0} \longrightarrow \operatorname{coker}(\Phi) \longrightarrow 0 .
$$

Ceci démontre 1-.

Supposons maintenant que les hypothèses de 2- soient vérifiées. Soit

$\mathcal{B}=(\Gamma \otimes \operatorname{Hom}(\Gamma, \mathcal{F})) \oplus \mathcal{F}^{\prime}$. On considère le morphisme injectif $B: \mathcal{E}^{\prime} \longrightarrow \mathcal{B}$ dont la première composante est la composée

$$
\mathcal{E}^{\prime} \stackrel{e v^{*}}{\longrightarrow} \Gamma \otimes \operatorname{Hom}\left(\mathcal{E}^{\prime}, \Gamma\right)^{*} \stackrel{\lambda}{\longrightarrow} \Gamma \otimes \operatorname{Hom}(\Gamma, \mathcal{F})
$$

et dont la seconde provient de $\Phi$. On a un diagramme commutatif avec lignes et colonnes exactes

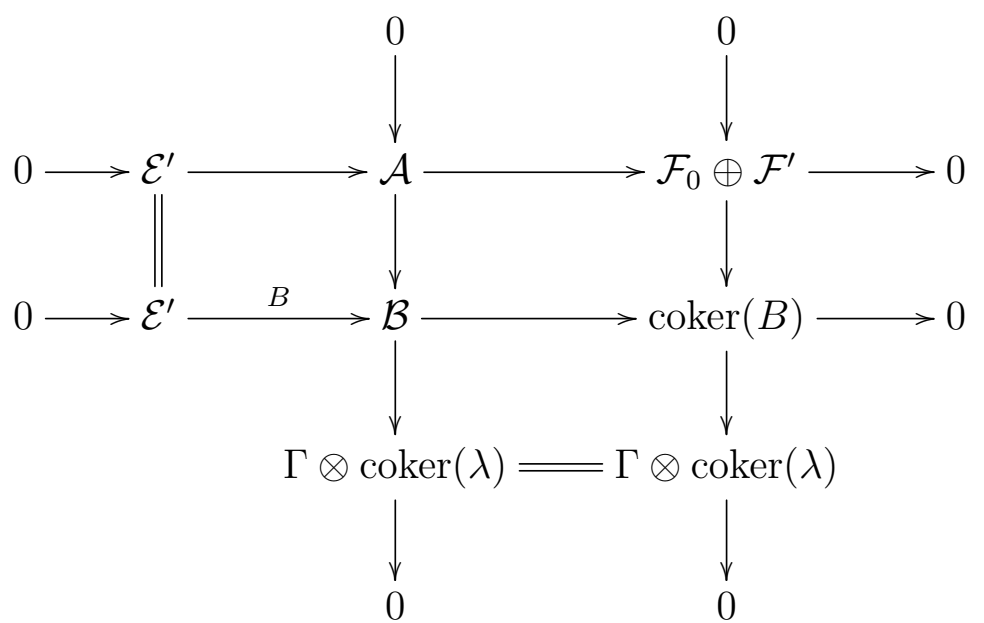

Puisque $\operatorname{Ext}^{1}\left(\Gamma, \mathcal{F}_{0}\right)=\operatorname{Ext}^{1}\left(\Gamma, \mathcal{F}^{\prime}\right)=\{0\}$, on a un isomorphisme

$$
\operatorname{coker}(B) \simeq(\Gamma \otimes \operatorname{coker}(\lambda)) \oplus \mathcal{F}_{0} \oplus \mathcal{F}^{\prime}
$$

Le carré commutatif

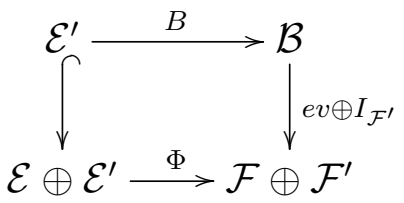

induit un morphisme surjectif $\rho: \operatorname{coker}(B) \longrightarrow \operatorname{coker}(\Phi)$, et une suite exacte

$$
0 \longrightarrow \mathcal{E}_{0} \longrightarrow \operatorname{ker}(\rho) \longrightarrow \mathcal{E} \longrightarrow 0 \text {. }
$$

Comme $\operatorname{Ext}^{1}\left(\mathcal{E}, \mathcal{E}_{0}\right)=\{0\}$, on a un isomorphisme $\operatorname{ker}(\rho) \simeq \mathcal{E} \oplus \mathcal{E}_{0}$. On a donc une suite exacte

$$
0 \longrightarrow \mathcal{E} \oplus \mathcal{E}_{0} \longrightarrow(\Gamma \otimes \operatorname{coker}(\lambda)) \oplus \mathcal{F}_{0} \oplus \mathcal{F}^{\prime} \longrightarrow \operatorname{coker}(\Phi) \longrightarrow 0
$$

Ceci démontre 2-. 


\subsection{Mutations de morphismes}

\subsubsection{Application de la proposition 3.1}

On va appliquer ce qui précède aux cas où $\mathcal{E}_{0}=0$ ou $\mathcal{F}_{0}=0$. Dans ce cas des symétries apparaissent qui justifient le changement de notations qu'on va effectuer. Soient $\mathcal{U}_{1}, \mathcal{U}_{2}, \mathcal{V}_{1}, \Gamma$ des faisceaux cohérents sur $\mathrm{X}$. On suppose que $\Gamma$ est simple et que le morphisme canonique

$$
e v^{*}: \mathcal{U}_{2} \longrightarrow \Gamma \otimes \operatorname{Hom}\left(\mathcal{U}_{2}, \Gamma\right)^{*}
$$

est injectif. On note $\mathcal{V}_{2}$ son conoyau. On suppose que

$$
\operatorname{Hom}\left(\Gamma, \mathcal{U}_{2}\right)=\operatorname{Ext}^{1}\left(\Gamma, \mathcal{U}_{2}\right)=\{0\}
$$

Ceci entraîne qu'on a un isomorphisme canonique

$$
\operatorname{Hom}\left(\Gamma, \mathcal{V}_{2}\right) \simeq \operatorname{Hom}\left(\mathcal{U}_{2}, \Gamma\right)^{*}
$$

et une suite exacte

$$
0 \longrightarrow \mathcal{U}_{2} \stackrel{e v^{*}}{\longrightarrow} \Gamma \otimes \operatorname{Hom}\left(\mathcal{U}_{2}, \Gamma\right)^{*} \simeq \Gamma \otimes \operatorname{Hom}\left(\Gamma, \mathcal{V}_{2}\right) \stackrel{e v}{\longrightarrow} \mathcal{V}_{2} \longrightarrow 0
$$

On suppose de plus que

$$
\operatorname{Ext}^{1}\left(\mathcal{U}_{1}, \mathcal{U}_{2}\right)=\operatorname{Ext}^{1}\left(\Gamma, \mathcal{V}_{1}\right)=\operatorname{Ext}^{1}\left(\mathcal{U}_{1}, \Gamma\right)=\operatorname{Ext}^{1}\left(\mathcal{V}_{2}, \mathcal{V}_{1}\right)=\{0\}
$$

Soient enfin $M, N$ des $\mathbb{C}$-espaces vectoriels de dimension finie. On a a alors le

Théorème 3.2. 1 - Soit $\Phi: \mathcal{U}_{1} \oplus \mathcal{U}_{2} \longrightarrow(\Gamma \otimes M) \oplus \mathcal{V}_{1}$ un morphisme injectif induisant une surjection $\lambda: \operatorname{Hom}\left(\mathcal{U}_{2}, \Gamma\right)^{*} \longrightarrow M$. Alors il existe un morphisme injectif

$$
\Phi^{\prime}: \mathcal{U}_{1} \oplus(\Gamma \otimes \operatorname{ker}(\lambda)) \longrightarrow \mathcal{V}_{2} \oplus \mathcal{V}_{1}
$$

tel que $\operatorname{coker}\left(\Phi^{\prime}\right) \simeq \operatorname{coker}(\Phi)$.

2 - Réciproquement soit $\Phi^{\prime}: \mathcal{U}_{1} \oplus(\Gamma \otimes N) \longrightarrow \mathcal{V}_{2} \oplus \mathcal{V}_{1}$ un morphisme injectif induisant une injection $\lambda: N \longrightarrow \operatorname{Hom}\left(\Gamma, \mathcal{V}_{2}\right)$. Alors il existe un morphisme injectif

$$
\Phi: \mathcal{U}_{1} \oplus \mathcal{U}_{2} \longrightarrow(\Gamma \otimes \operatorname{coker}(\lambda)) \oplus \mathcal{V}_{1}
$$

tel que $\operatorname{coker}(\Phi) \simeq \operatorname{coker}\left(\Phi^{\prime}\right)$.

Démonstration. Pour démontrer 1- on utilise la proposition 3.1, 1-, avec

$$
\mathcal{E}=\mathcal{U}_{1}, \mathcal{E}^{\prime}=\mathcal{U}_{2}, \mathcal{F}=\Gamma \otimes M, \mathcal{F}^{\prime}=\mathcal{V}_{1}
$$

Pour démontrer 2- on utilise la proposition 3.1, 2-, avec

$$
\mathcal{E}=\mathcal{U}_{1}, \mathcal{E}^{\prime}=\Gamma \otimes N, \mathcal{F}=\mathcal{V}_{2}, \mathcal{F}^{\prime}=\mathcal{V}_{1}
$$

On peut sans peine généraliser le résultat précédent à des morphismes non nécessairement injectifs (l'hypothèse d'injectivité simplifie les démonstrations). On associe en fait à un morphisme $\Phi$ un morphisme $\Phi^{\prime}$ ayant mêmes noyau et conoyau que $\Phi$. On dit que $\Phi^{\prime}$ est une mutation de $\Phi$ (et $\Phi$ une mutation de $\left.\Phi^{\prime}\right)$. 


\subsubsection{Description des mutations de morphismes}

On donne maintenant une description des transformations de morphismes dont il est question dans le théorème 3.2. Ceci a pour but de justifier les constructions abstraites du chapitre 4 . Formellement les deux transformations sont identiques (c'est encore plus clair si on suppose que les faisceaux $\mathcal{U}_{1}, \mathcal{U}_{2}, \mathcal{V}_{1}, \mathcal{V}_{2}$ et $\Gamma$ sont localement libres). On ne décrira que la première.

Soient $M, N$ des $\mathbb{C}$-espaces vectoriels tels que

$$
\operatorname{dim}(M)+\operatorname{dim}(N)=\operatorname{dim}\left(\operatorname{Hom}\left(\mathcal{U}_{2}, \Gamma\right)\right) .
$$

Soit

$$
\Phi: \mathcal{U}_{1} \oplus \mathcal{U}_{2} \longrightarrow(\Gamma \otimes M) \oplus \mathcal{V}_{1}
$$

un morphisme injectif, défini par la matrice

$$
\left(\begin{array}{ll}
\psi_{1} & \psi_{2} \\
\phi_{1} & \phi_{2}
\end{array}\right)
$$

avec

$$
\psi_{i}: \mathcal{U}_{i} \longrightarrow \Gamma \otimes M, \quad \phi_{i}: \mathcal{U}_{i} \longrightarrow \mathcal{V}_{1}
$$

pour $i=1,2$ (les notations suivent celles du chapitre 4 ). Soit

$$
\lambda: \operatorname{Hom}\left(\mathcal{U}_{2}, \Gamma\right)^{*} \longrightarrow M
$$

l'application linéaire déduite de $\phi_{2}$. On suppose que $\lambda$ est surjective. Soit

$$
A=\left(e v^{*}, \phi_{2}\right): \mathcal{U}_{2} \longrightarrow \mathcal{A}=\left(\Gamma \otimes \operatorname{Hom}\left(\mathcal{U}_{2}, \Gamma\right)^{*}\right) \oplus \mathcal{V}_{1},
$$

Comme dans la proposition 3.1, on montre que $\operatorname{coker}(A) \simeq \mathcal{V}_{2} \oplus \mathcal{V}_{1}$, en considérant le diagramme commutatif

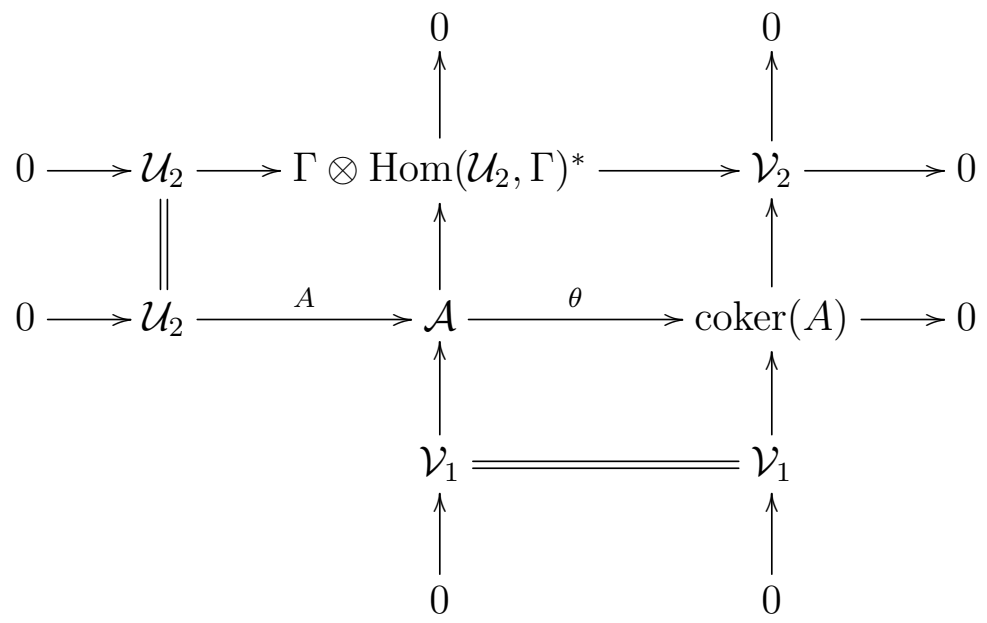

On a donc un morphisme

$$
\theta: \mathcal{A}=\left(\Gamma \otimes \operatorname{Hom}\left(\mathcal{U}_{2}, \Gamma\right)^{*}\right) \oplus \mathcal{V}_{1} \longrightarrow \mathcal{V}_{2} \oplus \mathcal{V}_{1}
$$

qu'on va décrire. D'après le diagramme commutatif précédent, la composante

$$
\theta_{22}: \Gamma \otimes \operatorname{Hom}\left(\mathcal{U}_{2}, \Gamma\right)^{*} \longrightarrow \mathcal{V}_{2}
$$

est le morphisme canonique, la composante $\mathcal{V}_{1} \longrightarrow \mathcal{V}_{2}$ est nulle, et la composante

$$
\theta_{1}: \mathcal{A}=\left(\Gamma \otimes \operatorname{Hom}\left(\mathcal{U}_{2}, \Gamma\right)^{*}\right) \oplus \mathcal{V}_{1} \longrightarrow \mathcal{V}_{1}
$$


est de la forme $\left(u, I_{\mathcal{V}_{1}}\right)$, où $u \in \operatorname{Hom}\left(\mathcal{U}_{2}, \Gamma\right) \otimes \operatorname{Hom}\left(\Gamma, \mathcal{V}_{1}\right)$. On a $\left(u, I_{\mathcal{V}_{1}}\right) \circ A=0$, d'où il découle que l'image de $u$ par le morphisme de composition

$$
\sigma: \operatorname{Hom}\left(\mathcal{U}_{2}, \Gamma\right) \otimes \operatorname{Hom}\left(\Gamma, \mathcal{V}_{1}\right) \longrightarrow \operatorname{Hom}\left(\mathcal{U}_{2}, \mathcal{V}_{1}\right)
$$

est $-\phi_{2}$. Réciproquement, étant donné $u^{\prime} \in \operatorname{Hom}\left(\mathcal{U}_{2}, \Gamma\right) \otimes \operatorname{Hom}\left(\Gamma, \mathcal{V}_{1}\right)$ dont l'image dans $\operatorname{Hom}\left(\mathcal{U}_{2}, \mathcal{V}_{1}\right)$ est $-\phi_{2}$, le morphisme

$$
\Gamma \otimes \operatorname{Hom}\left(\mathcal{U}_{2}, \Gamma\right)^{*} \longrightarrow \mathcal{V}_{2} \oplus \mathcal{V}_{1}
$$

défini par la matrice $\left(\begin{array}{cc}\theta_{22} & 0 \\ u^{\prime} & I_{\mathcal{V}_{1}}\end{array}\right)$ induit un isomorphisme $\operatorname{coker}(A) \simeq \mathcal{V}_{2} \oplus \mathcal{V}_{1}$, qui diffère du précédent par l'action de l'élément de $\operatorname{Aut}\left(\mathcal{V}_{2} \oplus \mathcal{V}_{1}\right)$ défini par la matrice $\left(\begin{array}{cc}I_{\mathcal{V}_{2}} & 0 \\ u-u^{\prime} & I_{\mathcal{V}_{1}}\end{array}\right)$, $u-u^{\prime}$ étant vu comme un élément de $\operatorname{ker}(\sigma) \simeq \operatorname{Hom}\left(\mathcal{V}_{2}, \mathcal{V}_{1}\right)$. On va maintenant décrire le morphisme

$$
\Phi^{\prime}: \mathcal{U}_{1} \oplus(\Gamma \otimes N) \longrightarrow \mathcal{V}_{2} \oplus \mathcal{V}_{1}
$$

associé à $\Phi$. Il est donné par la matrice

$$
\left(\begin{array}{ll}
\phi_{2}^{\prime} & \psi_{2}^{\prime} \\
\phi_{1}^{\prime} & \psi_{1}^{\prime}
\end{array}\right)
$$

avec

$$
\psi_{i}^{\prime}: \Gamma \otimes N \longrightarrow \mathcal{V}_{i}, \quad \phi_{i}^{\prime}: \mathcal{U}_{1} \longrightarrow \mathcal{V}_{i}
$$

pour $i=1,2$. On fixe un isomorphisme $\operatorname{ker}(\lambda) \simeq N$. Le morphisme $\Gamma \otimes N \longrightarrow \mathcal{V}_{2} \oplus \mathcal{V}_{1}$ induit par $\Phi^{\prime}$ provient du diagramme commutatif

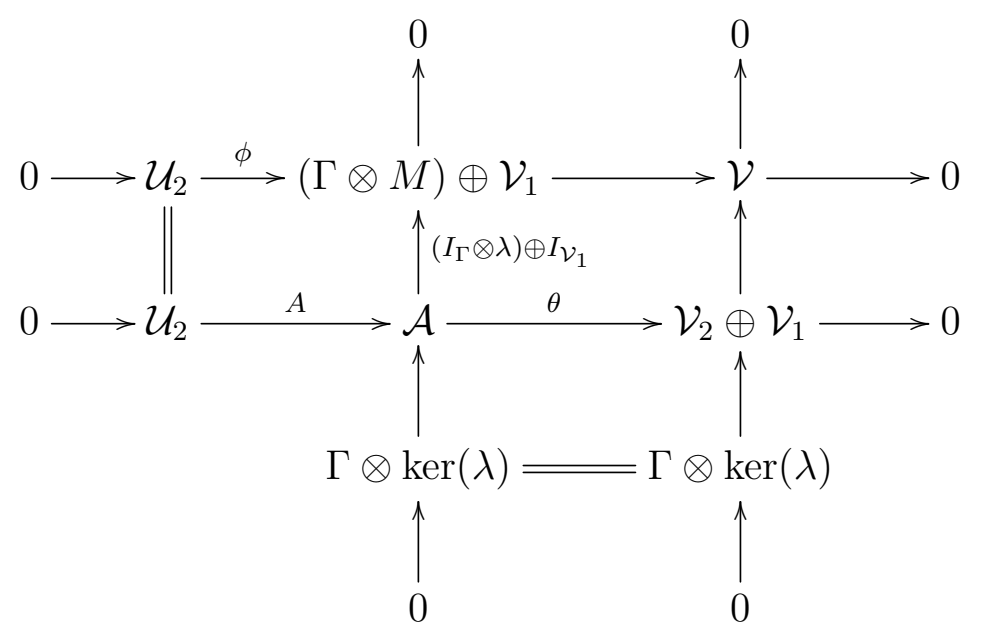

(avec $\mathcal{V}=\operatorname{coker}(\phi), \phi$ étant défini par $\phi_{2}$ et $\psi_{2}$ ). Il en découle que le morphisme

$$
\psi_{2}^{\prime}: \Gamma \otimes N \longrightarrow \mathcal{V}_{2}
$$

est induit par l'inclusion $N \subset \operatorname{Hom}\left(\mathcal{U}_{2}, \Gamma\right)^{*}=\operatorname{Hom}\left(\Gamma, \mathcal{V}_{2}\right)$. Le morphisme

$$
\psi_{1}^{\prime}: \Gamma \otimes N \longrightarrow \mathcal{V}_{1}
$$

est la restriction de $u: \Gamma \otimes \operatorname{Hom}\left(\mathcal{U}_{2}, \Gamma\right)^{*} \longrightarrow \mathcal{V}_{1}$. Il reste à définir

$$
\left(\phi_{1}^{\prime}, \phi_{2}^{\prime}\right): \mathcal{U}_{1} \longrightarrow \mathcal{V}_{1} \oplus \mathcal{V}_{2}
$$

On part du morphisme composé 


$$
\mathcal{U}_{1} \stackrel{\left(\psi_{1}, \phi_{1}\right)}{\longrightarrow}(\Gamma \otimes M) \oplus \mathcal{V}_{1} \longrightarrow \mathcal{V}
$$

(le second morphisme étant le morphisme quotient). Comme $\operatorname{Ext}^{1}\left(\mathcal{U}_{1}, \Gamma\right)=\operatorname{Ext}^{1}\left(\mathcal{U}_{1}, \mathcal{U}_{2}\right)=\{0\}$, ce morphisme se relève en un morphisme $\left(\phi_{1}^{\prime}, \phi_{2}^{\prime}\right): \mathcal{U}_{1} \longrightarrow \mathcal{V}_{1} \oplus \mathcal{V}_{2}$, qui lui-même se relève en un morphisme

$$
\Psi: \mathcal{U}_{1} \longrightarrow \mathcal{A}=\left(\Gamma \otimes \operatorname{Hom}\left(\mathcal{U}_{2}, \Gamma\right)^{*}\right) \oplus \mathcal{V}_{1}
$$

On peut décrire ces relèvements en utilisant le diagramme commutatif précédent. La composante $v: \mathcal{U}_{1} \longrightarrow \Gamma \otimes \operatorname{Hom}\left(\mathcal{U}_{2}, \Gamma\right)^{*}$ est un relèvement de $\psi_{1}: \mathcal{U}_{1} \longrightarrow \Gamma \otimes M$, et $\phi_{2}^{\prime}$ est la composition

$$
\mathcal{U}_{1} \stackrel{v}{\longrightarrow} \Gamma \otimes \operatorname{Hom}\left(\mathcal{U}_{2}, \Gamma\right)^{*}=\Gamma \otimes \operatorname{Hom}\left(\Gamma, \mathcal{V}_{2}\right) \stackrel{e v}{\longrightarrow} \mathcal{V}_{2}
$$

Le morphisme $\phi_{1}^{\prime}$ est la composition

$$
\mathcal{U}_{1} \stackrel{\Psi}{\longrightarrow} \mathcal{A} \stackrel{\theta_{1}}{\longrightarrow} \mathcal{V}_{1}
$$

On a donc

$$
\phi_{1}^{\prime}=<u, v>+\phi_{1},
$$

où $\langle u, v\rangle$ est obtenu de la façon suivante : rappelons que $u \in \operatorname{Hom}\left(\Gamma, \mathcal{V}_{1}\right) \otimes \operatorname{Hom}\left(\mathcal{U}_{2}, \Gamma\right)$ et $v \in \operatorname{Hom}\left(\mathcal{U}_{1}, \Gamma\right) \otimes \operatorname{Hom}\left(\mathcal{U}_{2}, \Gamma\right)^{*}$. La contraction de $u$ et $v$ donne donc un élément de $\operatorname{Hom}\left(\Gamma, \mathcal{V}_{1}\right) \otimes \operatorname{Hom}\left(\mathcal{U}_{1}, \Gamma\right)$, et $\langle u, v>$ est obtenu par composition.

\subsection{Application aux morphismes de type $(r, s)$}

Soient $X$ une variété projective, $r, s$ des entiers positifs, et $\mathcal{E}_{1}, \ldots, \mathcal{E}_{r}, \mathcal{F}_{1}, \ldots, \mathcal{F}_{s}$ des faisceaux cohérents simples sur $X$ tels que

$$
\begin{gathered}
\operatorname{Hom}\left(\mathcal{E}_{i}, \mathcal{E}_{i^{\prime}}\right)=0 \text { si } i>i^{\prime}, \operatorname{Hom}\left(\mathcal{F}_{j}, \mathcal{F}_{j^{\prime}}\right)=0 \text { si } j>j^{\prime}, \\
\operatorname{Hom}\left(\mathcal{F}_{j}, \mathcal{E}_{i}\right)=\{0\} \text { pour tous } i, j .
\end{gathered}
$$

On suppose que pour $1 \leq i \leq r$ le morphisme canonique

$$
\mathcal{E}_{i} \longrightarrow \operatorname{Hom}\left(\mathcal{E}_{i}, \mathcal{F}_{1}\right)^{*} \otimes \mathcal{F}_{1}
$$

est injectif. Soit $\mathcal{G}_{i}$ son conoyau. On suppose que

$$
\operatorname{Ext}^{1}\left(\mathcal{F}_{1}, \mathcal{F}_{j}\right)=\operatorname{Ext}^{1}\left(\mathcal{F}_{1}, \mathcal{E}_{i}\right)=\operatorname{Ext}^{1}\left(\mathcal{E}_{i}, \mathcal{F}_{1}\right)=\operatorname{Ext}^{1}\left(\mathcal{E}_{i}, \mathcal{E}_{k}\right)=\operatorname{Ext}^{1}\left(\mathcal{G}_{i}, \mathcal{F}_{j}\right)=\{0\}
$$

si $1 \leq i \leq k \leq r, 2 \leq j \leq s$. Du théorème 3.2 on déduit le

Théorème 3.3. Soient $M_{1}, \ldots, M_{r}, N_{1}, \ldots, N_{l}$ des $\mathbb{C}$-espaces vectoriels de dimension finie et $p$ un entier tel que $0 \leq p \leq r-1$.

1 - Soient

$$
\Phi: \bigoplus_{1 \leq i \leq r}\left(\mathcal{E}_{i} \otimes M_{i}\right) \longrightarrow \bigoplus_{1 \leq l \leq s}\left(\mathcal{F}_{l} \otimes N_{l}\right)
$$

un morphisme injectif et

$$
f_{p}: \bigoplus_{p+1 \leq j \leq r}\left(\operatorname{Hom}\left(\mathcal{E}_{j}, \mathcal{F}_{1}\right)^{*} \otimes M_{j}\right) \longrightarrow N_{1}
$$


l'application linéaire déduite de $\Phi$. On suppose que $f_{p}$ est surjective. Alors il existe un morphisme injectif

$$
\Phi^{\prime}:\left(\bigoplus_{1 \leq i \leq p}\left(\mathcal{E}_{i} \otimes M_{i}\right)\right) \oplus\left(\mathcal{F}_{1} \otimes \operatorname{ker}\left(f_{p}\right)\right) \longrightarrow\left(\bigoplus_{p<j \leq r}\left(\mathcal{G}_{j} \otimes M_{j}\right)\right) \oplus\left(\bigoplus_{2 \leq l \leq s}\left(\mathcal{F}_{l} \otimes N_{l}\right)\right)
$$

tel que $\operatorname{coker}\left(\Phi^{\prime}\right) \simeq \operatorname{coker}(\Phi)$.

2 - Soient $P_{1}$ un espace vectoriel de dimension finie,

$$
\Psi^{\prime}:\left(\bigoplus_{1 \leq i \leq p}\left(\mathcal{E}_{i} \otimes M_{i}\right)\right) \oplus\left(\mathcal{F}_{1} \otimes P_{1}\right) \longrightarrow\left(\bigoplus_{p<j \leq r}\left(\mathcal{G}_{j} \otimes M_{j}\right)\right) \oplus\left(\bigoplus_{2 \leq l \leq s}\left(\mathcal{F}_{l} \otimes N_{l}\right)\right)
$$

un morphisme injectif et

$$
g: P_{1} \longrightarrow \bigoplus_{p+1 \leq j \leq r}\left(\operatorname{Hom}\left(\mathcal{F}_{1}, \mathcal{G}_{j}\right) \otimes M_{j}\right)
$$

l'application linéaire déduite de $\Psi$. On suppose g injective. Alors il existe un morphisme injectif

$$
\Psi: \bigoplus_{1 \leq i \leq r}\left(\mathcal{E}_{i} \otimes M_{i}\right) \longrightarrow\left(\mathcal{F}_{1} \otimes \operatorname{coker}(g)\right) \oplus\left(\bigoplus_{2 \leq l \leq s}\left(\mathcal{F}_{l} \otimes N_{l}\right)\right)
$$

tel que $\operatorname{coker}(\Psi) \simeq \operatorname{coker}\left(\Psi^{\prime}\right)$.

Bien sûr on peut donner comme dans le $\S 3.2 .2$ une description complète des transformations faisant passer de $\Phi$ à $\Phi^{\prime}$ et de $\Phi^{\prime}$ à $\Phi$.

\section{Mutations abstraites}

\subsection{Espaces abstraits de morphismes}

\subsubsection{Motivation}

On décrit ici de façon abstraite le théorème 3.2, c'est-à-dire la transformation qui fait passer des morphismes du type

$$
\mathcal{U}_{1} \oplus \mathcal{U}_{2} \longrightarrow(\Gamma \otimes M) \oplus \mathcal{V}_{1}
$$

aux morphismes du type

$$
\mathcal{U}_{1} \oplus(\Gamma \otimes N) \longrightarrow \mathcal{V}_{2} \oplus \mathcal{V}_{1}
$$

(avec $\operatorname{dim}(M)+\operatorname{dim}(N)=\operatorname{dim}\left(\operatorname{Hom}\left(\mathcal{E}^{\prime}, \Gamma\right)\right)$, ainsi que la transformation réciproque. On dira qu'un de ces morphismes est une mutation de l'autre. On remarquera que formellement les deux types de morphismes sont semblables (c'est encore plus clair si les faisceaux en question sont localement libres et si on considère les transposés des morphismes du second type). D'autre part, une fois qu'on a réalisé cette similitude, il est facile de voir que la transformation inverse (d'un morphisme du second type à un morphisme du premier type) est formellement identique à la transformation directe. 
On supposera pour simplifier que

$$
\operatorname{Hom}\left(\mathcal{U}_{2}, \mathcal{U}_{1}\right)=\operatorname{Hom}\left(\Gamma, \mathcal{U}_{1}\right)=\operatorname{Hom}\left(\mathcal{V}_{1}, \mathcal{V}_{2}\right)=\operatorname{Hom}\left(\mathcal{V}_{1}, \Gamma\right)=\{0\}
$$

Soient

$$
W=\operatorname{Hom}\left(\mathcal{U}_{1} \oplus \mathcal{U}_{2},(\Gamma \otimes M) \oplus \mathcal{V}_{1}\right), \quad W^{\prime}=\operatorname{Hom}\left(\mathcal{U}_{1} \oplus(\Gamma \otimes N), \mathcal{V}_{2} \oplus \mathcal{V}_{1}\right)
$$

Soient $W_{0}$ l'ouvert de $W$ constitué des morphismes induisant une surjection $\operatorname{Hom}\left(\mathcal{U}_{2}, \Gamma\right)^{*} \longrightarrow M$, et $W_{0}^{\prime}$ l'ouvert de $W^{\prime}$ constitué des morphismes induisant une injection $N \longrightarrow \operatorname{Hom}\left(\Gamma, \mathcal{V}_{2}\right)$. Soient $G_{R}$ le groupe agissant à droite sur $W$, constitué des automorphismes de $\mathcal{U}_{1} \oplus \mathcal{U}_{2}$, et $G_{L}$ le groupe agissant à gauche sur $W$ constitué des automorphismes de $(\Gamma \otimes M) \oplus \mathcal{V}_{1}$. Soit enfin $G=G_{R}^{o p} \times G_{L}$ qui agit de façon évidente sur $W$. Soit $G^{\prime}$ le groupe analogue agissant sur $W^{\prime}$. On va construire de manière abstraite une bijection entre $W_{0} / G$ et $W_{0}^{\prime} / G^{\prime}$ (théorème 4.7). En fait on montrera dans le théorème 4.11 qu'on obtient un quasi-isomorphisme fort $W_{0} / G \simeq W_{0}^{\prime} / G^{\prime}$ (cf [4], §2). Dans les applications aux morphismes de type $(r, s)$ et sous certaines conditions on obtient en fait l'existence de bons quotients d'ouverts $G$-invariants de $W_{0}$ à partir de celle de bons quotients d'ouverts $G^{\prime}$-invariants de $W_{0}^{\prime}$ (cf. chapitre 5).

Une des raisons pour lesquelles une traduction abstraite du théorème 3.2 est utile est la suivante : il est théoriquement possible que toutes les hypothèses du $\S 3.2$ ne soient pas vérifiées par les faisceaux intervenant dans les morphismes du premier type, et qu'on ne puisse donc pas construire des mutations de ces morphismes comme morphismes du second type, mais que les mutations soient cependant définies de manière abstraite (comme éléments d'un espace vectoriel $W^{\prime}$ associé à $W$, obtenu comme indiqué dans ce qui va suivre). Mais je n'ai pas encore trouvé d'exemple réellement intéressant de cette situation.

\section{Plan de la suite du chapitre 4:}

Le reste du $\S 4.1$ est consacré à la définition d'un espace abstrait de morphismes. C'est une structure assez compliquée qui comporte en particulier la donnée d'un espace vectoriel $W$ appelé espace total sur lequel agit un groupe $G$. La correspondance entre les espaces abstraits de morphismes et les morphismes de faisceaux étudiés précédemment est donnée au § 4.1.6.

Dans les $\S 4.2$ et 4.3 on définit la mutation d'un espace abstrait de morphismes, qui est un autre espace abstrait de morphismes, comportant un autre espace total $W^{\prime}$ sur lequel agit un groupe $G^{\prime}$. Ce n'est rien d'autre que la formalisation de la description du $\S 3.2 .2$. On en déduit une bijection $W_{0} / G \simeq W_{0}^{\prime} / G^{\prime}, W_{0}$ étant un ouvert $G$-invariant particulier de $W$ et $W_{0}^{\prime}$ un ouvert $G^{\prime}$-invariant de $W^{\prime}$. Ceci généralise et améliore le théorème 3.2 .

Dans la suite du chapitre 4 on s'intéresse à la construction de bons quotients par des groupes algébriques. Dans ce cas on montre que la bijection précédente est en fait un quasi-isomorphisme fort. Cette notion est rappelée au \$ 4.4.2, ainsi que des résultats de [4] concernant les quasimorphismes et les quasi-morphismes forts entre variétés sur lesquelles opèrent des groupes algébriques.

L'étude des quasi-isomorphismes forts et leur utilisation pour construire des bons quotients est poursuivie dans le $\S 4.4 .3$. Les résultats du $\S 4.4 .3$ seront utilisés au chapitre 5 pour déduire l'existence d'un bon quotient par $G^{\prime}$ d'un ouvert $G^{\prime}$-invariant de $W_{0}^{\prime}$ de celle d'un bon quotient par $G$ de l'ouvert $G$-invariant correspondant de $W_{0}$, dans le cas des morphismes de type $(r, s)$. 
On prouve au $\S 4.4 .4$ que la bijection $W_{0} / G \simeq W_{0}^{\prime} / G^{\prime}$ est un quasi-isomorphisme fort (théorème 4.11.

On applique ce résultat au $\S 4.4 .4$ à la construction de quasi-bons quotients d'ouverts $G^{\prime}$ invariants de $W_{0}^{\prime}$ à partir de quasi-bons quotients d'ouverts $G$-invariants de $W_{0}$ (la notion de quasi-bon quotient est rappelée au § 4.4.2). On utilise uniquement ici le fait que la bijection précédente est un quasi-isomorphisme, ce qui est plus facile à démontrer que le théorème 4.11 . Dans le contexte des morphismes de type $(r, s)$ on peut cependant obtenir des bons quotients en utilisant ce théorème et les résultats du $\S 4.4 .3$.

\subsubsection{Notations}

Soit $k$ un corps commutatif. Pour tout espace $k$-vectoriel $K$, on note $\operatorname{tr}_{K}: K \otimes K^{*} \longrightarrow \mathbb{C}$ le morphisme trace. Si $E, F$ sont des $k$-espaces vectoriels, $\phi \in E \otimes K, \psi \in K^{*} \otimes F$, on notera

$$
<\phi \otimes \psi>_{K}=<\phi, \psi>_{K}=\left(I_{E \otimes F} \otimes t r_{K}\right)(\phi \otimes \psi)
$$

(s'il n'y a pas d'ambiguïté). On notera aussi $\langle\phi \otimes \psi\rangle$ ou $\langle\phi, \psi\rangle$ s'il n'y a pas de risque de confusion.

On note $L(E, F)$ l'espace vectoriel des applications linéaires de $E$ dans $F$.

\subsubsection{Définition générale}

Soient $\mathcal{N}_{1}, \mathcal{N}_{2}, \mathcal{M}_{1}, \mathcal{M}_{2}, M, \mathcal{A}_{0}, \mathcal{B}_{0}$ des espaces vectoriels sur $k$, de dimension finie, avec $\operatorname{dim}(M)<\operatorname{dim}\left(\mathcal{N}_{2}\right)$. On pose

$$
W=\left(\mathcal{N}_{1} \otimes M\right) \oplus\left(\mathcal{N}_{2} \otimes M\right) \oplus \mathcal{M}_{1} \oplus \mathcal{M}_{2}
$$

Les éléments de $W$ seront généralement représentés par des matrices $\left(\begin{array}{cc}\psi_{1} & \psi_{2} \\ \phi_{1} & \phi_{2}\end{array}\right)$, avec $\phi_{i} \in \mathcal{M}_{i}, \psi_{i} \in \mathcal{N}_{i} \otimes M$ pour $i=1,2$.

Soient $\mathbf{L}, \mathbf{R}, \mathbf{B}$ des groupes. On suppose que :

$\mathbf{L}$ opère linéairement à gauche sur $\mathcal{M}_{1}, \mathcal{M}_{2}, \mathcal{B}_{0}$.

$\mathbf{R}$ opère linéairement à droite sur $\mathcal{N}_{1}, \mathcal{M}_{1}, \mathcal{A}_{0}$.

$\mathrm{B}$ opère linéairement à droite sur $\mathcal{N}_{2}, \mathcal{M}_{2}$, et à gauche sur $\mathcal{A}_{0}$.

Les notations s'expliquent ainsi : le groupe $\mathbf{L}$ n'agit qu'à gauche ( $\mathbf{L}$ pour Left), $\mathbf{R}$ qu' à droite ( $\mathbf{R}$ pour Right), et $\mathbf{B}$ des deux cotés (B pour Both). Avec les notations du $\S 4.1 .1, \mathbf{L}, \mathbf{R}, \mathbf{B}$ jouent le rôle de $\operatorname{Aut}\left(\mathcal{V}_{1}\right)$, $\operatorname{Aut}\left(\mathcal{U}_{1}\right)$ et $\operatorname{Aut}\left(\mathcal{U}_{2}\right)$ respectivement.

On suppose que ces actions sont compatibles, c'est-à-dire que si deux de ces groupes $G_{\alpha}, G_{\beta}$, opèrent sur un même espace vectoriel $Z$, à gauche et à droite respectivement, pour tous $g_{\alpha} \in G_{\alpha}, g_{\beta} \in G_{\beta}$ et $z \in Z$ on a $g_{\alpha}\left(z g_{\beta}\right)=\left(g_{\alpha} z\right) g_{\beta}$. On suppose aussi que le groupe $\{1,-1\}$ est contenu dans $\mathbf{L}, \mathbf{R}$ et $\mathbf{B}$, et agit comme on le pense sur les espaces vectoriels sur lesquels ces groupes agissent (c'est-à-dire que -1 agit par multiplication par -1 ).

Soient

$$
\begin{aligned}
& \rho_{1}: \mathcal{B}_{0} \otimes \mathcal{N}_{1} \longrightarrow \mathcal{M}_{1}, \\
& \rho_{2}: \mathcal{B}_{0} \otimes \mathcal{N}_{2} \longrightarrow \mathcal{M}_{2},
\end{aligned}
$$




$$
\begin{aligned}
\nu: \mathcal{N}_{2} \otimes \mathcal{A}_{0} & \longrightarrow \mathcal{N}_{1}, \\
\mu: \mathcal{M}_{2} \otimes \mathcal{A}_{0} & \longrightarrow \mathcal{M}_{1}
\end{aligned}
$$

des applications linéaires. On suppose que le diagramme suivant $(D)$ est commutatif :

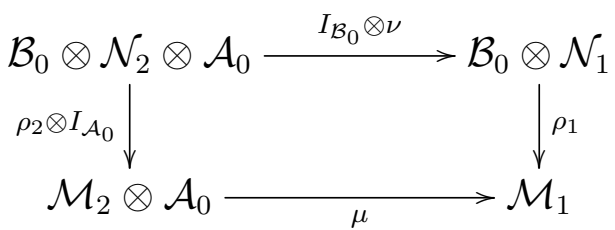

On suppose aussi que ces applications linéaires sont compatibles avec l'action des groupes. Par exemple $\mathbf{R}$ opère à droite sur $\mathcal{N}_{1}$ et $\mathcal{A}_{0}$, donc pour tous $\mathbf{r} \in \mathbf{R}, \boldsymbol{\alpha}_{0} \in \mathcal{A}_{0}$ et $l_{2} \in \mathcal{N}_{2}$ on a

$$
\nu\left(l_{2} \otimes\left(\boldsymbol{\alpha}_{0} \mathbf{r}\right)\right)=\nu\left(l_{2} \otimes \boldsymbol{\alpha}_{0}\right) . \mathbf{r} .
$$

De même, $\mathbf{B}$ opère à droite sur $\mathcal{N}_{2}$ et à gauche sur $\mathcal{A}_{0}$, donc pour tous $\mathbf{b} \in \mathbf{B}, \boldsymbol{\alpha}_{0} \in \mathcal{A}_{0}$ et $l_{2} \in \mathcal{N}_{2}$ on a

$$
\nu\left(l_{2} \mathbf{b} \otimes \boldsymbol{\alpha}_{0}\right)=\nu\left(l_{2} \otimes \mathbf{b} \boldsymbol{\alpha}_{0}\right) .
$$

On suppose aussi que $\rho_{2}$ est surjective, et que l'application linéaire

$$
\bar{\nu}: \mathcal{A}_{0} \longrightarrow \mathcal{N}_{2}^{*} \otimes \mathcal{N}_{1}
$$

déduite de $\nu$ est injective.

Définition 1. On appelle espace abstrait de morphismes et on note $\Theta$ la donnée de $\mathcal{N}_{1}, \mathcal{M}_{1}$, $\mathcal{N}_{2}, \mathcal{M}_{2}, \mathcal{A}_{0}, \mathcal{B}_{0}, M, \mathbf{L}, \mathbf{R}, \mathbf{B}, \nu, \mu, \rho_{1}$ et $\rho_{2}$. L'espace vectoriel

$$
W=\left(\mathcal{N}_{1} \otimes M\right) \oplus\left(\mathcal{N}_{2} \otimes M\right) \oplus \mathcal{M}_{1} \oplus \mathcal{M}_{2}
$$

est l'espace total de $\Theta$.

\subsubsection{Groupes associés}

On va construire deux nouveaux groupes associés à $\Theta: G_{L}$ et $G_{R}$. Le groupe $G_{R}$ est constitué des matrices $\left(\begin{array}{cc}\mathbf{r} & 0 \\ \boldsymbol{\alpha}_{0} & \mathbf{b}\end{array}\right)$ avec $\mathbf{r} \in \mathbf{R}, \mathbf{b} \in \mathbf{B}, \boldsymbol{\alpha}_{0} \in \mathcal{A}_{0}$. La loi de groupe de $G_{R}$ est

$$
\left(\begin{array}{cc}
\mathbf{r} & 0 \\
\boldsymbol{\alpha}_{0} & \mathbf{b}
\end{array}\right) \cdot\left(\begin{array}{cc}
\mathbf{r}^{\prime} & 0 \\
\boldsymbol{\alpha}_{0}^{\prime} & \mathbf{b}^{\prime}
\end{array}\right)=\left(\begin{array}{cc}
\mathbf{r r}^{\prime} & 0 \\
\boldsymbol{\alpha}_{0} \mathbf{r}^{\prime}+\mathbf{b} \boldsymbol{\alpha}_{0}^{\prime} & \mathbf{b} \mathbf{b}^{\prime}
\end{array}\right)
$$

Le groupe $G_{L}$ est constitué des matrices $\left(\begin{array}{cc}g_{M} & 0 \\ \beta & \ell\end{array}\right)$ avec $g_{M} \in G L(M), \boldsymbol{\ell} \in \mathbf{L}, \beta \in M^{*} \otimes \mathcal{B}_{0}$. La loi de groupe de $G_{L}$ est

$$
\left(\begin{array}{cc}
g_{M} & 0 \\
\beta & \ell
\end{array}\right) \cdot\left(\begin{array}{cc}
g_{M}^{\prime} & 0 \\
\beta^{\prime} & \ell^{\prime}
\end{array}\right)=\left(\begin{array}{cc}
g_{M} g_{M}^{\prime} & 0 \\
\beta g_{M}^{\prime}+\ell \beta^{\prime} & \boldsymbol{\ell} \ell^{\prime}
\end{array}\right)
$$

$\left(G L(M)\right.$ agit de manière évidente à droite sur le premier facteur de $M^{*} \otimes \mathcal{B}_{0}$, et $\mathbf{L}$ à gauche sur le deuxième facteur). 
4.1.5. Actions des groupes associés sur l'espace de morphismes

Le groupe $G_{R}$ opère à droite sur $W:$ si $\psi_{1} \in \mathcal{N}_{1} \otimes M, \psi_{2} \in \mathcal{N}_{2} \otimes M, \phi_{1} \in \mathcal{M}_{1}, \phi_{2} \in \mathcal{M}_{2}, \mathbf{r} \in \mathbf{R}$, $\mathbf{b} \in \mathbf{B}$ et $\boldsymbol{\alpha}_{0} \in \mathcal{A}_{0}$ on a

$$
\left(\begin{array}{cc}
\psi_{1} & \psi_{2} \\
\phi_{1} & \phi_{2}
\end{array}\right)\left(\begin{array}{cc}
\mathbf{r} & 0 \\
\boldsymbol{\alpha}_{0} & \mathbf{b}
\end{array}\right)=\left(\begin{array}{cc}
\psi_{1} \mathbf{r}+\left(\nu \otimes I_{M}\right)\left(\psi_{2} \otimes \boldsymbol{\alpha}_{0}\right) & \psi_{2} \mathbf{b} \\
\phi_{1} \mathbf{r}+\mu\left(\phi_{2} \otimes \boldsymbol{\alpha}_{0}\right) & \phi_{2} \mathbf{b}
\end{array}\right) .
$$

Le groupe $G_{L}$ opère à gauche sur $W$ : si $\psi_{1} \in \mathcal{N}_{1} \otimes M, \psi_{2} \in \mathcal{N}_{2} \otimes M, \phi_{1} \in \mathcal{M}_{1}, \phi_{2} \in \mathcal{M}_{2}$, $\boldsymbol{\ell} \in \mathbf{L}, g_{M} \in G L(M)$ et $\beta \in M^{*} \otimes \mathcal{B}_{0}$ on a

$$
\left(\begin{array}{cc}
g_{M} & 0 \\
\beta & \ell
\end{array}\right)\left(\begin{array}{cc}
\psi_{1} & \psi_{2} \\
\phi_{1} & \phi_{2}
\end{array}\right)=\left(\begin{array}{cc}
\left(I_{\mathcal{N}_{1}} \otimes g_{M}\right)\left(\psi_{1}\right) & \left(I_{\mathcal{N}_{2}} \otimes g_{M}\right)\left(\psi_{2}\right) \\
\boldsymbol{\ell} \phi_{1}+\rho_{1}\left(<\beta, \psi_{1}>_{M}\right) & \boldsymbol{\ell} \phi_{2}+\rho_{2}\left(<\beta, \psi_{2}>_{M}\right)
\end{array}\right) .
$$

Les actions de ces groupes sont compatibles, c'est-à-dire que si $g_{L} \in G_{L}, g_{R} \in G_{R}$ et $w \in W$, on a $g_{L}\left(w g_{R}\right)=\left(g_{L} w\right) g_{R}$. On obtient donc une action à gauche du groupe

$$
G=G_{R}^{o p} \times G_{L}
$$

sur $W$.

On note $H$ le sous-groupe de $G$ constitué des paires

$$
\left(\left(\begin{array}{cc}
1 & 0 \\
\boldsymbol{\alpha}_{0} & 1
\end{array}\right),\left(\begin{array}{cc}
1 & 0 \\
\beta & 1
\end{array}\right)\right)
$$

(où $\boldsymbol{\alpha}_{0} \in \mathcal{A}_{0}, \beta \in M^{*} \otimes \mathcal{B}_{0}$ ).

\subsubsection{Dictionnaire}

Soient $\Gamma, \mathcal{U}_{1}, \mathcal{U}_{2}, \mathcal{V}_{1}$ et $\mathcal{V}_{2}$ des faisceaux cohérents sur $X$ possédant les propriétés du $\S 3.2 .1$ et $M$ un $\mathbb{C}$-espace vectoriel non nul dels que $\operatorname{dim}(M) \leq \operatorname{dim}\left(\operatorname{Hom}\left(\mathcal{U}_{2}, \Gamma\right)\right.$. On en déduit un espace abstrait de morphismes qu'on décrit ci-dessous. On considère

$$
\begin{gathered}
\mathcal{M}_{1}=\operatorname{Hom}\left(\mathcal{U}_{1}, \mathcal{V}_{1}\right), \quad \mathcal{M}_{2}=\operatorname{Hom}\left(\mathcal{U}_{2}, \mathcal{V}_{1}\right), \quad \mathcal{A}_{0}=\operatorname{Hom}\left(\mathcal{U}_{1}, \mathcal{U}_{2}\right), \\
\mathcal{N}_{1}=\operatorname{Hom}\left(\mathcal{U}_{1}, \Gamma\right), \quad \mathcal{N}_{2}=\operatorname{Hom}\left(\mathcal{U}_{2}, \Gamma\right), \quad \mathcal{B}_{0}=\operatorname{Hom}\left(\Gamma, \mathcal{V}_{1}\right) .
\end{gathered}
$$

Les applications $\rho_{1}, \rho_{2}, \mu$ et $\nu$ sont les compositions. On a

$$
\mathbf{B}=\operatorname{Aut}\left(\mathcal{U}_{2}\right), \quad \mathbf{L}=\operatorname{Aut}\left(\mathcal{V}_{1}\right), \quad \mathbf{R}=\operatorname{Aut}\left(\mathcal{U}_{1}\right)
$$

opérant de façon évidente sur les espaces vectoriels précédents. L'espace total de cet espace abstrait de morphismes est

$$
W=\operatorname{Hom}\left(\mathcal{U}_{1} \oplus \mathcal{U}_{2},(\Gamma \otimes M) \oplus \mathcal{V}_{1}\right)
$$

Si de plus on suppose que

$$
\operatorname{Hom}\left(\mathcal{U}_{2}, \mathcal{U}_{1}\right)=\operatorname{Hom}\left(\Gamma, \mathcal{U}_{1}\right)=\operatorname{Hom}\left(\mathcal{V}_{1}, \mathcal{V}_{2}\right)=\operatorname{Hom}\left(\mathcal{V}_{1}, \Gamma\right)=\{0\}
$$

on a $G_{L}=\operatorname{Aut}\left((\Gamma \otimes M) \oplus \mathcal{V}_{1}\right), G_{R}=\operatorname{Aut}\left(\mathcal{U}_{1} \oplus \mathcal{U}_{2}\right)$ opérant eux aussi de la façon évidente. 


\subsection{Mutation d'un espace abstrait de morphismes}

Soit $N$ un $k$-espace vectoriel tel que $\operatorname{dim}(N)=\operatorname{dim}\left(\mathcal{N}_{2}\right)-\operatorname{dim}(M)$. On va définir un nouvel espace abstrait de morphismes $D(\Theta)$ associé à $\Theta$.

\subsubsection{Les espaces vectoriels}

Posons

$$
\mathcal{N}_{1}^{\prime}=\mathcal{B}_{0}, \quad \mathcal{N}_{2}^{\prime}=\mathcal{N}_{2}^{*}, \quad \mathcal{M}_{1}^{\prime}=\mathcal{M}_{1}, \quad \mathcal{B}_{0}^{\prime}=\mathcal{N}_{1} .
$$

On définit $\mathcal{M}_{2}^{\prime}$ et $\mathcal{A}_{0}^{\prime}$ par les suites exactes

$$
\begin{array}{r}
0 \longrightarrow \mathcal{A}_{0}^{\prime} \longrightarrow \mathcal{B}_{0} \otimes \mathcal{N}_{2} \stackrel{\rho_{2}}{\longrightarrow} \mathcal{M}_{2} \longrightarrow 0, \\
0 \longrightarrow \mathcal{A}_{0} \stackrel{\bar{\nu}}{\longrightarrow} \mathcal{N}_{2}{ }^{*} \otimes \mathcal{N}_{1} \longrightarrow \mathcal{M}_{2}^{\prime} \longrightarrow 0
\end{array}
$$

\subsubsection{Les morphismes}

Lemme 4.1. L'application

$$
\rho_{1} \circ \operatorname{tr}_{\mathcal{N}_{2}}: \mathcal{B}_{0} \otimes \mathcal{N}_{2} \otimes \mathcal{N}_{2}^{*} \otimes \mathcal{N}_{1} \longrightarrow \mathcal{M}_{1}
$$

s'annule sur $\mathcal{A}_{0}^{\prime} \otimes \mathcal{A}_{0}$.

Démonstration. Le morphisme $\nu$ se factorise de la façon suivante :

$$
\mathcal{N}_{2} \otimes \mathcal{A}_{0} \stackrel{\bar{\nu}}{\longrightarrow} \mathcal{N}_{2} \otimes \mathcal{N}_{2}^{*} \otimes \mathcal{M}_{1} \stackrel{{ }^{t \mathcal{N}_{2}} \otimes I}{\longrightarrow} \mathcal{M}_{1}
$$

On en déduit avec le diagramme $(D)$ un autre diagramme commutatif :

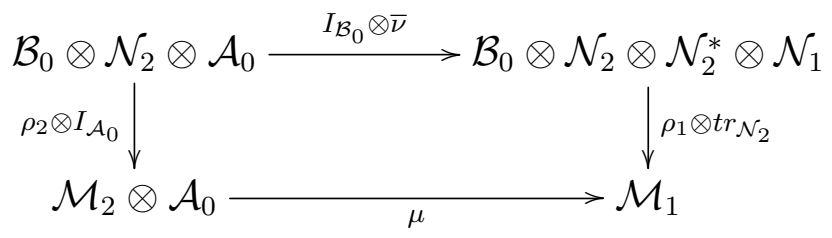

On en déduit immédiatement le lemme.

Lemme 4.2. Soient

$$
\begin{aligned}
& 0 \longrightarrow X_{0} \stackrel{i^{\prime}}{\longrightarrow} E \otimes K \stackrel{i^{\prime}}{\longrightarrow} X^{0} \longrightarrow 0, \\
& 0 \longrightarrow Y_{0} \stackrel{i^{\prime \prime}}{\longrightarrow} K^{*} \otimes F \stackrel{\longrightarrow}{\longrightarrow} Y^{0} \text { ? }
\end{aligned}
$$

des suites exactes d'espaces vectoriels. Soit $\phi: E \otimes F \longrightarrow C$ une application linéaire telle que

$$
\phi \circ\left(I_{E} \otimes \operatorname{tr}_{K} \otimes I_{F}\right): E \otimes K \otimes K^{*} \otimes F \longrightarrow C
$$

s'annule sur $X_{0} \otimes Y_{0}$. Soient

$$
\begin{aligned}
& r^{\prime}=\left(I_{E} \otimes t r_{K} \otimes I_{F}\right) \circ\left(I_{E \otimes K} \otimes i^{\prime \prime}\right): E \otimes K \otimes Y_{0} \longrightarrow E \otimes F, \\
& r^{\prime \prime}=\left(I_{E} \otimes t r_{K} \otimes I_{F}\right) \circ\left(i^{\prime} \otimes I_{K^{*} \otimes F}\right): E \otimes K \otimes Y_{0} \longrightarrow E \otimes F .
\end{aligned}
$$

Alors il existe des applications linéaires uniques

$$
\phi^{\prime}: X^{0} \otimes Y_{0} \longrightarrow C, \quad \phi^{\prime \prime}: X_{0} \otimes Y^{0} \longrightarrow C
$$


telles qu'on ait un diagramme commutatif

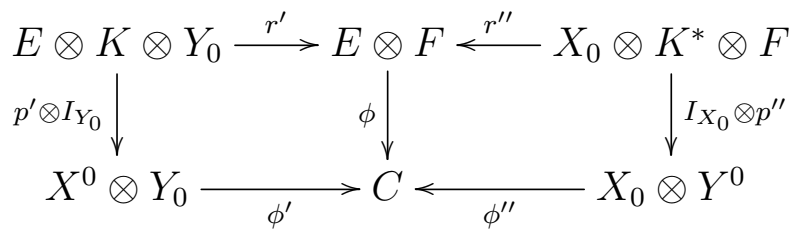

Démonstration. L'unicité de $\phi^{\prime}$ découle de la surjectivité de $p^{\prime}$. Son existence découle du fait que $\phi \circ r^{\prime}$ s'annule sur $\operatorname{ker}\left(p^{\prime} \otimes I_{Y_{0}}\right)=X_{0} \otimes Y_{0}$. L'existence et l'unicité de $\phi^{\prime \prime}$ sont analogues.

On applique les lemmes précédents aux suites exactes du 4.2.1. Le diagramme de gauche est (D) (cf 4.1.3), et celui de droite, écrit convenablement, est $\left(D^{\prime}\right)$ :

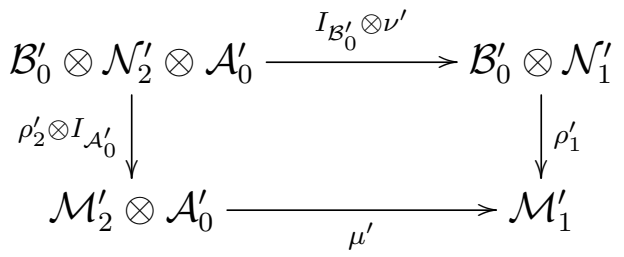

ce qui définit les applications $\nu^{\prime}, \rho_{2}^{\prime}, \rho_{1}^{\prime}$ et $\mu^{\prime}$.

Les morphismes $\nu^{\prime}, \rho_{2}^{\prime}, \rho_{1}^{\prime}$ sont définis plus simplement de la façon suivante :

$$
\rho_{1}^{\prime}=\rho_{1} \text {. }
$$

$\rho_{2}^{\prime}$ est la projection $\mathcal{N}_{2}^{*} \otimes \mathcal{N}_{1} \longrightarrow\left(\mathcal{N}_{2}^{*} \otimes \mathcal{N}_{1}\right) / \bar{\nu}\left(\mathcal{A}_{0}\right)$.

$\overline{\nu^{\prime}}$ est l'inclusion $\operatorname{ker}\left(\rho_{2}\right) \subset \mathcal{B}_{0} \otimes \mathcal{N}_{2}$.

\subsubsection{Les groupes}

On pose $\mathbf{L}^{\prime}=\mathbf{R}^{o p}, \quad \mathbf{R}^{\prime}=\mathbf{L}^{o p}, \quad \mathbf{B}^{\prime}=\mathbf{B}^{o p}$. Les actions de ces groupes se déduisent immédiatement de celles des groupes $\mathbf{L}, \mathbf{R}$ et $\mathbf{B}$. Par exemple, $\mathbf{R}$ agit à droite sur $\mathcal{A}_{0}$ et $\mathcal{N}_{1}$, et cette action est compatible avec $\nu: \mathcal{N}_{2} \otimes \mathcal{A}_{0} \longrightarrow \mathcal{N}_{1}$. On obtient donc une action à droite de $\mathbf{R}$ sur $\left(\mathcal{N}_{2}{ }^{*} \otimes \mathcal{N}_{1}\right) / \mathcal{A}_{0}$, c'est-à-dire une action à gauche de $\mathbf{L}^{\prime}$ sur $\mathcal{M}_{2}^{\prime}$.

\subsubsection{Mutation d'un espace abstrait de morphismes}

Définition 2. On note $D(\Theta)$ l'espace abstrait de morphismes défini par $\mathcal{N}_{1}^{\prime}, \mathcal{N}_{2}^{\prime}, \mathcal{M}_{1}^{\prime}, \mathcal{M}_{2}^{\prime}, \mathcal{A}_{0}^{\prime}$, $\mathcal{B}_{0}^{\prime}, N^{*}, \mathbf{L}^{\prime}, \mathbf{R}^{\prime}, \mathbf{B}^{\prime}, \nu^{\prime}, \mu^{\prime}, \rho_{1}^{\prime}$ et $\rho_{2}{ }^{\prime}$. On l'appelle la mutation de $\Theta$.

Proposition 4.3. On a $D(D(\Theta))=\Theta$.

Immédiat. Cela découle notamment de la symétrie du diagramme commutatif du lemme 4.2 ,

On définit comme pour $\Theta$ les groupes $G^{\prime}=G_{R}^{\prime o p} \times G_{L}^{\prime}$ et $H^{\prime}$ correspondant à $D(\Theta)$. 


\subsubsection{Dictionnaire}

Avec les notations du $\S 3.2 .1$, dans la situation du $\S 4.1 .6$ on a

$$
\begin{gathered}
\mathcal{M}_{1}^{\prime}=\operatorname{Hom}\left(\mathcal{U}_{1}, \mathcal{V}_{1}\right), \quad \mathcal{M}_{2}^{\prime}=\operatorname{Hom}\left(\mathcal{U}_{1}, \mathcal{V}_{2}\right), \quad \mathcal{A}_{0}=\operatorname{Hom}\left(\mathcal{V}_{2}, \mathcal{V}_{1}\right), \\
\mathcal{N}_{1}^{\prime}=\operatorname{Hom}\left(\Gamma, \mathcal{V}_{1}\right), \quad \mathcal{N}_{2}^{\prime}=\operatorname{Hom}\left(\Gamma, \mathcal{V}_{2}\right), \quad \mathcal{B}_{0}^{\prime}=\operatorname{Hom}\left(\mathcal{U}_{1}, \Gamma\right) .
\end{gathered}
$$

Les applications $\rho_{1}^{\prime}, \rho_{2}^{\prime}, \mu^{\prime}$ et $\nu^{\prime}$ sont les compositions.

En ce qui concerne les groupes la situation est un peu plus compliquée. Pour obtenir une représentation exacte il faudrait supposer que tous les faisceaux qui interviennent sont localement libres et que les mutations sont des morphismes $\mathcal{V}_{1}^{*} \oplus \mathcal{V}_{2}^{*} \longrightarrow\left(\Gamma^{*} \otimes N^{*}\right) \oplus \mathcal{U}_{1}^{*}$. Si on néglige cette subtilité on a

$$
\mathbf{B}^{\prime}=\operatorname{Aut}\left(\mathcal{V}_{2}\right), \quad \mathbf{L}^{\prime}=\operatorname{Aut}\left(\mathcal{U}_{1}\right), \quad \mathbf{R}^{\prime}=\operatorname{Aut}\left(\mathcal{V}_{1}\right)
$$

opérant de façon évidente sur les espaces vectoriels précédents. L'espace total de cet espace abstrait de morphismes est

$$
W^{\prime}=\operatorname{Hom}\left(\mathcal{U}_{1} \oplus(\Gamma \otimes N), \mathcal{V}_{2} \oplus \mathcal{V}_{1}\right)
$$

On a $G_{L}^{\prime}=\operatorname{Aut}\left(\mathcal{U}_{1} \oplus(\Gamma \otimes N)\right), G_{R}^{\prime}=\operatorname{Aut}\left(\mathcal{V}_{2} \oplus \mathcal{V}_{1}\right)$ opérant eux aussi de la façon évidente.

\subsection{Mutation des morphismes}

\subsubsection{Définition}

On note $W^{\prime}$ l'espace total de $D(\Theta)$, c'est-à-dire

$$
W^{\prime}=\left(\mathcal{N}_{1}^{\prime} \otimes N^{*}\right) \oplus\left(\mathcal{N}_{2}^{\prime} \otimes N^{*}\right) \oplus \mathcal{M}_{1}^{\prime} \oplus \mathcal{M}_{2}^{\prime} .
$$

On note $W^{0}$ l'ouvert de $W$ constitué des $\left(\begin{array}{ll}\psi_{1} & \psi_{2} \\ \phi_{1} & \phi_{2}\end{array}\right)$ tels que l'application linéaire $\overline{\psi_{2}}: \mathcal{N}_{2}{ }^{*} \longrightarrow M$ déduite de $\psi_{2}$ soit surjective. On définit de même l'ouvert $W^{\prime 0}$ de $W^{\prime}$.

Soit

$$
w=\left(\begin{array}{cc}
\psi_{1} & \psi_{2} \\
\phi_{1} & \phi_{2}
\end{array}\right) \in W^{0}
$$

On va en déduire un élément de $W^{\prime 0}$ (pas de manière unique). On choisit d'abord un isomorphisme

$$
\operatorname{ker}\left(\overline{\psi_{2}}\right) \simeq N
$$

On note $\psi_{2}^{\prime}$ l'élément de $\mathcal{N}_{2}^{\prime} \otimes N^{*}$ provenant de l'application linéaire

$$
\overline{\psi_{2}^{\prime}}: \mathcal{N}_{2}^{\prime *}=\mathcal{N}_{2} \longrightarrow \operatorname{ker}\left(\overline{\psi_{2}}\right)^{*}=N^{*},
$$

qui est la transposée de l'inclusion de $\operatorname{ker}\left(\overline{\psi_{2}}\right)$ dans $\mathcal{N}_{2}{ }^{*}$.

On considère le diagramme commutatif avec ligne exacte :

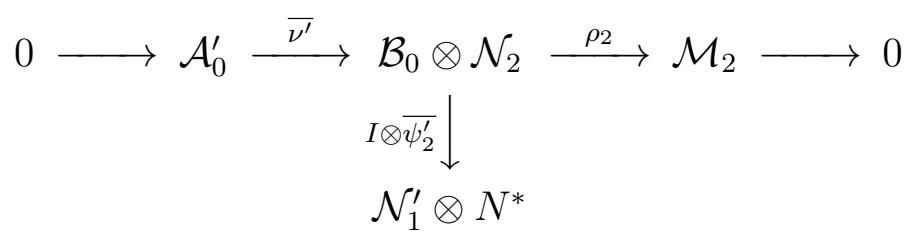


Soient

$$
u \in \rho_{2}^{-1}\left(-\phi_{2}\right) \subset \mathcal{B}_{0} \otimes \mathcal{N}_{2}, \quad \text { et } \quad \psi_{1}^{\prime}=\left(I \otimes \overline{\psi_{2}^{\prime}}\right)(u) \in \mathcal{N}_{1}^{\prime} \otimes N^{*} .
$$

Notons que $u$ est défini à un élément près de $\mathcal{A}_{0}^{\prime}$.

On considère le diagramme analogue au précédent, mais correspondant à $D(\Theta)$.

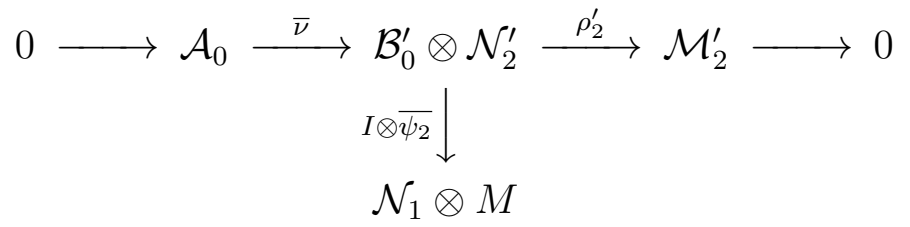

Soient

$$
v \in\left(I \otimes \overline{\psi_{2}}\right)^{-1}\left(\psi_{1}\right) \quad \subset \mathcal{B}_{0}^{\prime} \otimes \mathcal{N}_{2}^{\prime}, \quad \text { et } \quad \phi_{2}^{\prime}=\rho_{2}^{\prime}(v) \in \mathcal{M}_{2}^{\prime} .
$$

Notons que $v$ est défini à un élément près de $N \otimes \mathcal{B}_{0}^{\prime}$.

Soient enfin

$$
\phi_{1}^{\prime}=\phi_{1}+\rho_{1}\left(<v, u>_{\mathcal{N}_{2}}\right), \quad \text { et } \quad z(w, u, v)=\left(\begin{array}{cc}
\psi_{1}^{\prime} & \psi_{2}^{\prime} \\
\phi_{1}^{\prime} & \phi_{2}^{\prime}
\end{array}\right) \in W^{\prime 0}
$$

On emploiera aussi la notation $z(w)=z(w, u, v)$, bien que cet élément de $W^{\prime 0}$ ne dépende pas uniquement de $w$.

\subsubsection{Propriétés}

Proposition 4.4. Soit $w \in W^{0}$. Les éléments $z(w, u, v)$, pour tous les choix possibles de $u$ et $v$, constituent une $H^{\prime}$-orbite de $W^{\prime 0}$.

Démonstration. On vérifie aisément que si on remplace $u$ par $u+\boldsymbol{\alpha}_{0}^{\prime}$ et $v$ par $v+\beta^{\prime}$ (avec $\boldsymbol{\alpha}_{0}^{\prime} \in \mathcal{A}_{0}^{\prime}$ et $\left.\beta^{\prime} \in N \otimes \mathcal{B}_{0}^{\prime}\right)$, l'élément obtenu de $W^{\prime}$ est

$$
\left(\begin{array}{cc}
1 & 0 \\
\beta^{\prime} & 1
\end{array}\right)\left(\begin{array}{ll}
\psi_{1}^{\prime} & \psi_{2}^{\prime} \\
\phi_{1}^{\prime} & \phi_{2}^{\prime}
\end{array}\right)\left(\begin{array}{cc}
1 & 0 \\
\boldsymbol{\alpha}_{0}^{\prime} & 1
\end{array}\right) .
$$

Proposition 4.5. Pour tout $w \in W^{0}$, on a $z(z(w)) \in G w$.

Démonstration. On part de

$$
w=\left(\begin{array}{cc}
\psi_{1} & \psi_{2} \\
\phi_{1} & \phi_{2}
\end{array}\right) \in W^{0}
$$

et on prend comme précédemment $u \in \rho_{2}^{-1}\left(-\phi_{2}\right), v \in\left(I \otimes \overline{\psi_{2}}\right)^{-1}\left(\psi_{1}\right)$ pour définir

$$
z(w)=\left(\begin{array}{cc}
\psi_{1}^{\prime} & \psi_{2}^{\prime} \\
\phi_{1}^{\prime} & \phi_{2}^{\prime}
\end{array}\right) \in W^{\prime 0} .
$$

On cherche maintenant $u^{\prime} \in \rho_{2}{ }^{-1}\left(-\phi_{2}^{\prime}\right)$ et $v^{\prime} \in\left(I \otimes \overline{\psi_{2}^{\prime}}\right)^{-1}\left(\psi_{1}^{\prime}\right)$ pour définir $z(z(w))$. On a $\rho_{2}{ }^{\prime}(-v)=-\phi_{2}^{\prime}$, donc on peut prendre $u^{\prime}=-v$. D'autre part, on a $\left(I \otimes \overline{\psi_{2}^{\prime}}\right)(u)=\psi_{1}^{\prime}$, et on peut prendre $v^{\prime}=u$. 
Soit

$$
z(z(w))=\left(\begin{array}{cc}
\psi_{1}^{\prime \prime} & \psi_{2}^{\prime \prime} \\
\phi_{1}^{\prime \prime} & \phi_{2}^{\prime \prime}
\end{array}\right) \in W^{0}
$$

l'élément de $W^{0}$ défini par $u^{\prime}$ et $v^{\prime}$. On a évidemment $\psi_{2}^{\prime \prime}=\psi_{2}$, et

$$
\begin{gathered}
\psi_{1}^{\prime \prime}=\left(I \otimes \overline{\psi_{2}}\right)\left(u^{\prime}\right)=-\left(I \otimes \overline{\psi_{2}}\right)(v)=-\psi_{1}, \\
\phi_{2}^{\prime \prime}=\rho_{2}\left(v^{\prime}\right)=\rho_{2}(u)=-\phi_{2}, \\
\phi_{1}^{\prime \prime}=\phi_{1}^{\prime}+\rho_{1}\left(<v^{\prime}, u^{\prime}>_{\mathcal{N}_{2}}\right)=\phi_{1}+\rho_{1}\left(<v, u>_{\mathcal{N}_{2}}\right)-\rho_{1}\left(<v, u>_{\mathcal{N}_{2}}\right)=\phi_{1} .
\end{gathered}
$$

Donc

$$
\begin{aligned}
z(z(w)) & =\left(\begin{array}{cc}
-\psi_{1} & \psi_{2} \\
\phi_{1} & -\phi_{2}
\end{array}\right) \\
& =\left(\begin{array}{cc}
-1 & 0 \\
0 & 1
\end{array}\right)\left(\begin{array}{ll}
\psi_{1} & \psi_{2} \\
\phi_{1} & \phi_{2}
\end{array}\right)\left(\begin{array}{cc}
1 & 0 \\
0 & -1
\end{array}\right)
\end{aligned}
$$

Proposition 4.6. Soient $w_{1}, w_{2} \in W^{0}$ des points qui sont dans la même G-orbite. Alors $z\left(w_{1}\right)$ et $z\left(w_{2}\right)$ sont dans la même $G^{\prime}$-orbite.

Démonstration. On vérifie aisément que c'est vrai si

$$
w_{2}=\left(\begin{array}{cc}
g_{M} & 0 \\
0 & \ell
\end{array}\right) w_{1}\left(\begin{array}{cc}
\mathbf{r} & 0 \\
0 & \mathbf{b}
\end{array}\right) .
$$

Il reste à traiter les cas

$$
w_{2}=\left(\begin{array}{cc}
1 & 0 \\
\beta & 1
\end{array}\right) w_{1}, \quad \text { ou } \quad w_{2}=w_{1}\left(\begin{array}{cc}
1 & 0 \\
\boldsymbol{\alpha}_{0} & 1
\end{array}\right),
$$

avec $\boldsymbol{\alpha}_{0} \in \mathcal{A}_{0}, \beta \in M^{*} \otimes \mathcal{B}_{0}$. On ne traitera que le premier cas, le second étant analogue. Posons

$$
w_{1}=\left(\begin{array}{cc}
\psi_{1} & \psi_{2} \\
\phi_{1} & \phi_{2}
\end{array}\right) \text {. }
$$

Alors on a

$$
w_{2}=\left(\begin{array}{cc}
\psi_{1}+\left(\nu \otimes I_{M}\right)\left(\psi_{2} \otimes \boldsymbol{\alpha}_{0}\right) & \psi_{2} \\
\phi_{1}+\mu\left(\phi_{2} \otimes \boldsymbol{\alpha}_{0}\right) & \phi_{2}
\end{array}\right) .
$$

On suppose que

$$
z\left(w_{1}\right)=\left(\begin{array}{ll}
\psi_{1}^{\prime} & \psi_{2}^{\prime} \\
\phi_{1}^{\prime} & \phi_{2}^{\prime}
\end{array}\right)
$$

est défini par $u_{1} \in \mathcal{B}_{0} \otimes \mathcal{N}_{2}$ et $v_{1} \in \mathcal{N}_{2}{ }^{*} \otimes \mathcal{N}_{1}$. On va chercher des éléments $u_{2}, v_{2}$ convenables pour définir $z\left(w_{2}\right)$. On doit avoir $u_{2} \in \rho_{2}{ }^{-1}\left(-\phi_{2}\right)$, donc on peut prendre $u_{2}=u_{1}$. On doit avoir

$$
\left(I \otimes \overline{\psi_{2}}\right)\left(v_{2}\right)=\phi_{1}+\left(\nu \otimes I_{M}\right)\left(\psi_{2} \otimes \boldsymbol{\alpha}_{0}\right) .
$$

Posons $v_{2}=v_{1}+v_{0}$. On doit donc avoir

$$
\left(I \otimes \overline{\psi_{2}}\right)\left(v_{0}\right)=\left(\nu \otimes I_{M}\right)\left(\psi_{2} \otimes \boldsymbol{\alpha}_{0}\right) .
$$


Pour cela il suffit de prendre $v_{0}=\boldsymbol{\alpha}_{0}$ (vu comme élément de $\mathcal{N}_{2}{ }^{*} \otimes \mathcal{N}_{1}$, à l'aide de $\bar{\nu}$ ). On a alors

$$
z\left(w_{2}\right)=\left(\begin{array}{ll}
\psi_{1}^{\prime \prime} & \psi_{2}^{\prime} \\
\phi_{1}^{\prime \prime} & \phi_{2}^{\prime \prime}
\end{array}\right),
$$

avec

$$
\begin{gathered}
\psi_{1}^{\prime \prime}=\left(I \otimes \overline{\psi_{2}^{\prime}}\right)\left(u_{2}\right)=\left(I \otimes \overline{\psi_{2}^{\prime}}\right)\left(u_{1}\right)=\psi_{1}^{\prime}, \\
\phi_{2}^{\prime \prime}=\rho_{2}\left(v_{2}\right)=\rho_{2}\left(v_{1}+v_{0}\right)=\rho_{2}\left(v_{1}\right)=\phi_{2}^{\prime}, \\
\phi_{1}^{\prime \prime}=\phi_{1}+\rho_{1}\left(<v_{2}, u_{2}>_{\mathcal{N}_{2}}\right) \\
=\phi_{1}^{\prime}-\mu\left(\boldsymbol{\alpha}_{0} \otimes \rho_{2}\left(u_{2}\right)\right)+\rho_{1}\left(<v_{0}, u_{2}>_{\mathcal{N}_{2}}\right)
\end{gathered}
$$

Mais on a $\mu\left(\boldsymbol{\alpha}_{0} \otimes \rho_{2}\left(u_{2}\right)\right)=\rho_{1}\left(<v_{0}, u_{2}>_{\mathcal{N}_{2}}\right)$, si on se souvient que $v_{0}=\bar{\nu}\left(\boldsymbol{\alpha}_{0}\right)$, à cause du diagramme commutatif $(D)$ du $\S 4.1 .3$. On a donc $\phi_{1}^{\prime \prime}=\phi_{1}^{\prime}$ et finalement $z\left(w_{1}\right)=z\left(w_{2}\right)$.

\subsection{Théorèmes d'isomorphisme}

\subsubsection{Correspondance entre les ensembles d'orbites}

Le théorème suivant découle immédiatement des résultats des $\S 4.2$ et 4.3 :

Théorème 4.7. L'application associant à l'orbite d'un point $w$ de $W^{0}$ l'orbite de $z(w)$ définit une bijection

$$
D_{\Theta}: W^{0} / G \simeq W^{\prime 0} / G^{\prime}
$$

dont l'inverse est $D_{D(\Theta)}$.

\subsubsection{Quasi-isomorphismes et quasi-bons quotients}

Les définitions et résultats de cette partie proviennent de [4].

Soit $G$ un groupe algébrique. On appelle $G$-espace une variété algébrique $X$ munie d'une action algébrique de $G$. Rappelons qu'on appelle bon quotient de $X$ par $G$ un morphisme

$$
\pi: X \longrightarrow M
$$

(où $M$ est une variété algébrique) tel que :

(i) Le morphisme $\pi$ est $G$-invariant, affine et surjectif.

(ii) Si $U$ est un ouvert de $M$, alors on a $\mathcal{O}(U) \simeq \mathcal{O}\left(\pi^{-1}(U)\right)^{G}$.

(iii) Si $F_{1}, F_{2}$ sont des sous-variétés fermées $G$-invariantes disjointes de $X$, alors $\pi\left(F_{1}\right)$ et $\pi\left(F_{2}\right)$ sont des sous-variétés disjointes de $M$. 
Définition 3. On appelle quasi-bon quotient de $X$ par $G$ un morphisme

$$
\pi: X \longrightarrow M
$$

(où $M$ est une variété algébrique) qui est G-invariant, surjectif et tel que les conditions (ii) et (iii) précédentes soient vérifiées.

On dit parfois par abus de language que $M$ est le quasi-bon quotient de $X$ par $G$. On note comme dans le cas des bons quotients $M=X / / G$. Il est clair qu'un bon quotient est un quasi-bon quotient.

Soient $G, G^{\prime}$ des groupes algébriques, $X$ un $G$-espace et $X^{\prime}$ un $G^{\prime}$-espace.

Définition 4. 1 - On appelle quasi-morphisme de $X$ vers $X^{\prime}$ une application

$$
\phi: X / G \longrightarrow X^{\prime} / G^{\prime}
$$

telle que pour tout point $x$ de $X$ il existe un ouvert de Zariski $U$ de $X$ contenant $x$ et un morphisme $U \longrightarrow X^{\prime}$ induisant $\phi$. On appelle quasi-isomorphisme de $X$ vers $X^{\prime}$ une bijection $\phi: X / G \longrightarrow X^{\prime} / G^{\prime}$ qui est un quasi-morphisme ainsi que son inverse.

2 - On appelle quasi-morphisme fort de $X$ vers $X^{\prime}$ la donnée d'un quasi-morphisme

$\phi: X / G \longrightarrow X^{\prime} / G^{\prime}$, d'un recouvrement ouvert $\left(U_{i}\right)_{i \in I}$ de $X$, et d'une famille $\left(\phi_{i}\right)_{i \in I}$ de relèvements de $\phi, \phi_{i}: U_{i} \longrightarrow X^{\prime}$, telle que :

(i) pour tout $j \in I$ et $g \in G$, le morphisme

$$
\begin{gathered}
g U_{j} \longrightarrow X^{\prime} \\
x \longmapsto \phi_{j}\left(g^{-1} x\right)
\end{gathered}
$$

appartient à la famille $\left(\phi_{i}\right)$.

(ii) Pour tous $i, j \in I$, et tout $x \in U_{i} \cap U_{j}$ il existe un voisinage $V$ de $x$ dans $U_{i} \cap U_{j}$ et un morphisme $\lambda_{i j}: V \longrightarrow G^{\prime}$ tel que $\phi_{j \mid V}=\lambda_{i j} \phi_{i \mid V}$,

(iii) Pour tout $i \in I$ et $x \in U_{i}$, il existe un voisinage $V$ de $(e, x)$ dans $G \times U_{i}$ et un morphisme $\gamma: V \longrightarrow G^{\prime}$ tel que $\gamma(e, x)=e$ et que pour tous $(g, y) \in V$, on ait gy $\in U_{i}$ et $\phi_{i}(g y)=\gamma(g, y) \phi_{i}(y)$.

On appelle quasi-isomorphisme de $X$ vers $X^{\prime}$ une bijection $\phi: X / G \longrightarrow X^{\prime} / G^{\prime}$ qui est un quasi-morphisme ainsi que son inverse.

Il est clair que si $\phi: X / G \longrightarrow X^{\prime} / G^{\prime}$ est un quasi-isomorphisme (non nécessairement fort), $\phi$ induit une bijection entre l'ensemble des ouverts (resp. fermés) $G$-invariants de $X$ et l'ensemble des ouverts (resp. fermés) $G^{\prime}$-invariants de $X^{\prime}$. De plus, si $X$ est lisse, les hypersurfaces $G$ invariantes de $X$ correspondent aux hypersurfaces $G^{\prime}$-invariantes de $X^{\prime}$. Si $U$ est un ouvert $G$-invariant de $X$, et $U^{\prime}$ l'ouvert $G^{\prime}$-invariant correspondant de $X^{\prime}, \phi$ induit un isomorphisme d'anneaux

$$
\mathcal{O}(U)^{G} \simeq \mathcal{O}\left(U^{\prime}\right)^{G^{\prime}}
$$

Plus généralement, si $Y$ est une variété algébrique, les morphismes $G$-invariants $X \longrightarrow Y$ s'identifient de manière évidente aux morphismes $G^{\prime}$-invariants $X^{\prime} \longrightarrow Y$. 
Définition 5. Soit $\sigma=\left(\phi: X / G \longrightarrow X^{\prime} / G^{\prime},\left(\phi_{i}: U_{i} \longrightarrow X^{\prime}\right)\right)$ un quasi-morphisme fort. On appelle carte de $\sigma$ un relèvement local $f: U \longrightarrow X^{\prime}$ de $\phi$ ( $U$ étant un ouvert non vide de $X$ ) tel que pour tout $x \in U$ les propriétés suivantes soient vérifiées :

(i) Pour tout $i \in I$ il existe un voisinage $V$ de $x$ dans $U \cap U_{i}$ et un morphisme $\lambda_{i}: V \longrightarrow G^{\prime}$ tel que $f_{\mid V}=\lambda_{i} \phi_{i \mid V}$,

(ii) Il existe un voisinage $V$ de $(e, x)$ dans $G \times U$ et un morphisme $\gamma: V \longrightarrow G^{\prime}$ tel que $\gamma(e, x)=e$ et que pour tous $(g, y) \in V$, on ait $g y \in U$ et $\phi_{i}(g y)=\gamma(g, y) \phi(y)$.

On dit que deux quasi-morphismes forts de $X$ dans $X^{\prime}$ sont équivalents si les cartes de l'un sont aussi des cartes de l'autre. On définit aussi dans [4 la composition de deux quasimorphismes forts : si un quasi-morphisme fort de $X$ dans $X^{\prime}$ (resp. de $X^{\prime}$ vers $X^{\prime \prime}$ ) est défini $\operatorname{par}\left(\phi_{i}: U_{i} \longrightarrow X^{\prime}\right)_{i \in I}$ (resp. $\left.\left(\psi_{j}: V_{j} \longrightarrow X^{\prime \prime}\right)_{j \in J}\right)$, on définit un quasi-morphisme fort de $X$ vers $X^{\prime \prime}$ par $\left(\psi_{j} \circ \phi_{i}: \phi_{i}^{-1}\left(V_{j}\right) \longrightarrow X^{\prime \prime}\right)_{(i, j) \in I \times J}$. On définit de manière évidente le quasimorphisme fort identité $I_{X}$ de $X$ dans $X$. Il est clair que la composition à droite ou à gauche d'un quasi-morphisme fort avec $I_{X}$ donne un quasi-morphisme fort équivalent. On définit un quasi-isomorphisme fort $\sigma$ de $X$ dans $X^{\prime}$ comme étant un quasi-morphisme fort de $X$ dans $X^{\prime}$ tel qu'il existe un quasi-morphisme fort $\sigma^{\prime}$ de $X^{\prime}$ dans $X$ tel que $\sigma \circ \sigma^{\prime}$ (resp. $\sigma^{\prime} \circ \sigma$ ) soit équivalent à $I_{X^{\prime}}\left(\operatorname{resp} . I_{X}\right)$.

Proposition 4.8. Si $X$ et $X^{\prime}$ sont quasi-isomorphes, il existe un quasi-bon quotient de $X$ par $G$ si et seulement si il existe un quasi-bon quotient de $X^{\prime}$ par $G^{\prime}$, et dans ce cas les deux quotients sont isomorphes.

(prop. 2.2 de [4]).

Rappelons qu'un $G$-fibré vectoriel sur $X$ est un fibré vectoriel algébrique $F$ sur $X$ muni d'une action algébrique linéaire de $G$, au dessus de l'action de $G$ sur $X$.

Définition 6. On dit qu'un $G$-fibré vectoriel $F$ sur $X$ est admissible si pour tout point $x$ de $X$, le stabilisateur de $x$ dans $G$ agit trivialement sur $F_{x}$.

On suppose qu'il existe un quasi-isomorphisme fort entre $X$ et $X^{\prime}$. On montre dans [4], $\S 2.3$, qu'il existe alors une bijection canonique entre l'ensemble des classes d'isomorphisme de $G$-fibrés vectoriels admissibles sur $X$ et l'ensemble des classes d'isomorphisme de $G^{\prime}$-fibrés vectoriels admissibles sur $X^{\prime}$. De plus, il est aisé de voir que si $F$ est un $G$-fibré vectoriel admissible sur $X$ et $F^{\prime}$ le $G^{\prime}$-fibré vectoriel admissible sur $X^{\prime}$ correspondant, on a un isomorphisme canonique

$$
H^{0}(X, F)^{G} \simeq H^{0}\left(X^{\prime}, F^{\prime}\right)^{G^{\prime}} .
$$


4.4.3. Actions de groupes sur des espaces affines

Soient $G, G^{\prime}$ des groupes algébriques agissant linéairement sur des espaces vectoriels $E, E^{\prime}$ respectivement, de dimension finie positive. Soient $X$ un ouvert $G$-invariant de $E, X^{\prime}$ un ouvert $G^{\prime}$-invariant de $E^{\prime}$. On suppose donné un quasi-isomorphisme fort entre $X$ et $X^{\prime}$.

Soit $\chi$ un caractère non trivial de $G$. Soit $E^{s s}(\chi)$ l'ouvert de $E$ constitué des points $x$ tels qu'il existe un entier $k>0$ et un polynôme homogène $\chi^{k}$-invariant $f$ tel que $f(x) \neq 0$. On note $\mathcal{L}_{\chi}$ le $G$-fibré en droites sur $E$ induit par $\chi$ (le fibré en droites sous-jacent est trivial et l'action de $G$ induite par $\chi)$. Alors les polynômes $\chi^{k}$-invariants sont exactement les sections $G$-invariantes de $\mathcal{L}_{\chi}^{k}$, et $\mathcal{L}_{\chi}$ est admissible sur $E^{s s}(\chi)$. Si $f$ est un tel polynôme, on note $E_{f}$ l'ouvert de $E^{s s}(\chi)$ constitué des points où $f$ ne s'annule pas. On emploie des notations analogues pour $E^{\prime}$.

Proposition 4.9. On suppose qu'il existe un bon quotient $M$ de $E^{s s}(\chi)$ par $G$, et que les propriétés suivantes sont vérifiées :

(1) Toute fonction régulière inversible sur $G^{\prime}$ est le produit d'un caractère par une constante.

(2) On a $E^{s s}(\chi) \subset X$.

(3) On a $\operatorname{codim}\left(X \backslash E^{s s}(\chi)\right) \geq 2$.

(4) Il existe un recouvrement de $M$ par des ouverts affines dont l'image inverse dans $E^{s s}(\chi)$ soit de la forme $E_{f}$ (où $f$ est un polynôme $\chi^{k}$-invariant, pour un entier $k>0$ ).

Soit $X_{0}^{\prime}$ l'ouvert de $X^{\prime}$ correspondant à $E^{s s}(\chi)$. Alors le $G^{\prime}$-fibré en droites admissible sur $X_{0}^{\prime}$ correspondant à $\mathcal{L}_{\chi \mid E^{s s}(\chi)}$ est de la forme $\mathcal{L}_{\chi^{\prime} \mid X_{0}^{\prime}}$, on a $X_{0}^{\prime}=E^{\prime s s}\left(\chi^{\prime}\right)$ et le quasi-bon quotient $E^{\prime s s}\left(\chi^{\prime}\right) / / G$ est un bon quotient.

Démonstration. Il découle de la propriété 1 et de la proposition 14 de [2] que tout $G^{\prime}$-fibré en droites sur un ouvert $G^{\prime}$-invariant $U$ de $E^{\prime}$ est de la forme $\mathcal{L}_{\chi^{\prime} \mid U}$, pour un caractère $\chi^{\prime}$ de $G^{\prime}$. Ceci prouve la première assertion de la proposition 4.9.

Montrons que $X_{0}^{\prime}=E^{\prime s s}\left(\chi^{\prime}\right)$. Remarquons d'abord que $\operatorname{codim}\left(E^{\prime} \backslash X_{0}^{\prime}\right) \geq 2$. En effet dans le cas contraire $X^{\prime} \backslash X_{0}^{\prime}$ contiendrait une hypersurface de $X^{\prime}$ qui correspondrait à une hypersurface de $X \backslash E^{s s}(\chi)$, ce qui est impossible. Les sections $G^{\prime}$-invariantes de $\mathcal{L}_{\chi^{\prime} \mid X_{0}^{\prime}}$ se prolongent donc à $E^{\prime}$. Comme pour tout point $x$ de $E^{s s}(\chi)$ il existe un entier $k>0$ une section $G$-invariante de $\mathcal{L}_{\chi^{k}}$ ne s'annulant pas en $x$, on en déduit que pour tout point $x^{\prime}$ de $E^{\prime s s}\left(\chi^{\prime}\right)$ il existe un entier $k>0$ et une section $G^{\prime}$-invariante de $\mathcal{L}_{\chi^{\prime k}}$ ne s'annulant pas en $x^{\prime}$. On a donc $X_{0}^{\prime}=E^{\prime s s}\left(\chi^{\prime}\right)$.

Montrons maintenant que $M$ est un bon quotient de $X_{0}^{\prime}$ par $G^{\prime}$. Soit $U$ un ouvert affine non vide de $M$, dont l'image inverse dans $E^{s s}(\chi)$ est de la forme $E_{f}, f$ étant un polynôme $\chi^{k}$-invariant. Alors $f$ est une section $G$-invariante de $\mathcal{L}_{\chi^{k}}$. Soit $f^{\prime}$ la section $G^{\prime}$-invariante correspondante de $\mathcal{L}_{\chi^{\prime k}}$. Alors l'ouvert de $E^{\prime s s}\left(\chi^{\prime}\right)$ au dessus de $U$ n'est autre que $E_{f^{\prime}}^{\prime}$, qui est affine.

Remarques : Les propriétés de la proposition 4.9 vérifiées par le quotient $X^{s s}(\chi) \longrightarrow M$ le sont aussi par le quotient associé $X^{\prime s s}\left(\chi^{\prime}\right) \longrightarrow \bar{M}$.

Proposition 4.10. On suppose qu'il existe un bon quotient de $X$ par $G$ et que les propriétés suivantes sont vérifiées :

(1) Toute hypersurface G-invariante de E contient $E \backslash X$. 
(2) Toute hypersurface $G^{\prime}$-invariante de $E^{\prime}$ contient $E^{\prime} \backslash X^{\prime}$.

Alors le quasi-bon quotient $X^{\prime} / / G^{\prime} \simeq M$ est un bon quotient.

Démonstration. Soit $U$ un ouvert affine de $M, X_{0}$ l'ouvert affine de $X$ au dessus de $U$. Alors $X_{0}$ est le complémentaire d'une hypersurface $G$-invariante dans $E$. Il en découle que l'ouvert correspondant $X_{0}^{\prime}$ de $X^{\prime}$ est aussi le complémentaire d'une hypersurface $G^{\prime}$-invariante $H^{\prime}$ dans $X^{\prime}$. Comme l'adhérence $\overline{H^{\prime}}$ de cette hypersurface dans $E^{\prime}$ contient $E^{\prime} \backslash X^{\prime}$, on a $X_{0}^{\prime}=E^{\prime} \backslash \overline{H^{\prime}}$, qui est affine.

\subsubsection{Théorème d'isomorphisme fort}

On suppose maintenant que les groupes $\mathbf{L}, \mathbf{R}, \mathbf{B}$ sont algébriques sur $k$ et que leurs actions sont algébriques.

On reprend les notations des $\S 4.1,4.2$ et 4.3 et 4.4.1.

Théorème 4.11. La bijection

$$
D_{\Theta}: W^{0} / G \simeq W^{\prime 0} / G^{\prime}
$$

est un quasi-isomorphisme fort, ainsi que son inverse $D_{D(\Theta)}$.

Démonstration. La démonstration comporte six étapes.

Étape 1 - Définition de quelques applications linéaires

Soit

$$
r_{2}: \mathcal{M}_{2} \longrightarrow \mathcal{B}_{0} \otimes \mathcal{N}_{2}
$$

une application linéaire telle que $\rho_{2} \circ r_{2}=I_{\mathcal{M}_{2}}$ (son existence découle de la surjectivité de $\rho_{2}$ ).

Soit $M_{0}$ un sous-espace vectoriel de $\mathcal{N}_{2}^{*}$ tel que $\operatorname{dim}\left(M_{0}\right)=\operatorname{dim}(M)$. Soit $U_{2}\left(M_{0}\right)$ l'ouvert de $\mathcal{N}_{2} \otimes M$ constitué des applications linéaires surjectives $f: \mathcal{N}_{2}^{*} \longrightarrow M$ telles que $\operatorname{ker}(f) \cap M_{0}=\{0\}$. Soit $W\left(M_{0}\right)$ l'ouvert de $W$ constitué des éléments dont la composante de $\mathcal{N}_{2} \otimes M$ est dans $U_{2}\left(M_{0}\right)$.

Pour tout $\psi_{2} \in \mathcal{N}_{2} \otimes M$, on note $\overline{\psi_{2}}: \mathcal{N}_{2}^{*} \longrightarrow M$ l'application linéaire déduite de $\psi_{2}$. Si $\psi_{2} \in U_{2}\left(M_{0}\right), \bar{\psi}_{2} M_{0}$ est un isomorphisme $M_{0} \simeq M$, et on note $r_{M_{0}}\left(\psi_{2}\right)$ l'application linéaire $M \longrightarrow \mathcal{N}_{2}^{*}$ qui est la composée

$$
M \stackrel{\left(\overline{\psi_{2}} \mid M_{0}\right)^{-1}}{\longrightarrow} M_{0} \longrightarrow \mathcal{N}_{2}^{*} .
$$

On a $\overline{\psi_{2}} \circ r_{M_{0}}\left(\psi_{2}\right)=I_{M}$.

Soit maintenant

$$
\begin{array}{rlc}
s_{M_{0}}\left(\psi_{2}\right): \mathcal{N}_{2}^{*} & \longrightarrow & \operatorname{ker}\left(\overline{\psi_{2}}\right) \\
x & \longmapsto x-r_{M_{0}}\left(\psi_{2}\right) \circ \overline{\psi_{2}}(x)
\end{array}
$$

On a alors $s_{M_{0}}\left(\psi_{2}\right)_{\mid \operatorname{ker}\left(\overline{\psi_{2}}\right)}=I_{\mathrm{ker}\left(\overline{\psi_{2}}\right)}$ et

$$
<r_{M_{0}}\left(\psi_{2}\right) \otimes \psi_{2}>_{M}+<s_{M_{0}}\left(\psi_{2}\right) \otimes i_{\psi_{2}}>_{\operatorname{ker}\left(\overline{\psi_{2}}\right)}=I_{\mathcal{N}_{2}},
$$


$i_{\psi_{2}}$ désignant l'inclusion $\operatorname{ker}\left(\overline{\psi_{2}}\right) \longrightarrow \mathcal{N}_{2}^{*}$.

Soit $N_{0} \subset \mathcal{N}_{2}^{*}$ un sous-espace vectoriel supplémentaire de $M_{0}$. On fixe un isomorphisme $\epsilon_{0}: N \longrightarrow N_{0}$. La projection sur $N_{0}$ associée à la décomposition $\mathcal{N}_{2}=M_{0} \oplus N_{0}$ induit un isomorphisme $p: \operatorname{ker}\left(\overline{\psi_{2}}\right) \simeq N_{0}$. On note $q=q\left(M_{0}, N_{0}, \epsilon_{0}, \psi_{2}\right)=\epsilon_{0}^{-1} \circ p: \operatorname{ker}\left(\overline{\psi_{2}}\right) \simeq N$.

Étape 2 - Définition des cartes et du quasi-isomorphisme

Soit $I$ l'ensemble des triplets $\left(M_{0}, N_{0}, \epsilon_{0}\right)$ comme précédemment. On va définir un recouvrement ouvert $\left(U_{i}\right)_{i \in I}$ de $W_{0}$, et pour tout $i \in I$ un morphisme

$$
\lambda_{i}: U_{i} \longrightarrow W_{0}^{\prime}
$$

au dessus de $D_{\theta}$. Si $i=\left(M_{0}, N_{0}, \epsilon_{0}\right)$, on prend $U_{i}=W\left(M_{0}\right)$. Pour définir $\lambda_{i}$ on suit le $\S 4.3$ Soit

$$
w=\left(\begin{array}{cc}
\psi_{1} & \psi_{2} \\
\phi_{1} & \phi_{2}
\end{array}\right) \in W\left(M_{0}\right)
$$

avec $\psi_{1} \in \mathcal{N}_{1} \otimes M, \psi_{2} \in \mathcal{N}_{2} \otimes M, \phi_{1} \in \mathcal{M}_{1}, \phi_{2} \in \mathcal{M}_{2}$. Soit

$$
\overline{\psi_{2}^{\prime}}: \mathcal{N}_{2}^{\prime *}=\mathcal{N}_{2} \longrightarrow N^{*}
$$

l'application linéaire composée

$$
\mathcal{N}_{2} \longrightarrow \operatorname{ker}\left(\overline{\psi_{2}}\right)^{*} \stackrel{{ }^{t} q^{-1}}{\longrightarrow} N^{*}
$$

Soit $\psi_{2}^{\prime} \in \mathcal{N}_{2}^{\prime} \otimes N^{*}$ l'élément associé à $\overline{\psi_{2}^{\prime}}$. Soient $u=-r_{2}\left(\phi_{2}\right) \in \mathcal{B}_{0} \otimes \mathcal{N}_{2}$ et

$$
\psi_{1}^{\prime}=\left(I_{\mathcal{B}_{0}} \otimes \overline{\psi_{2}^{\prime}}\right)(u) \in \mathcal{N}_{1}^{\prime} \otimes N^{*} .
$$

Soient $v=\left(I \otimes r_{M_{0}}\left(\psi_{2}\right)\right)\left(\psi_{1}\right) \in \mathcal{B}_{0}^{\prime} \otimes \mathcal{N}_{2}^{\prime}$ et

$$
\phi_{2}^{\prime}=\rho_{2}^{\prime}(v) \in \mathcal{M}_{2}^{\prime}
$$

Soit enfin

On définit $\lambda_{i}$ par

$$
\phi_{1}^{\prime}=\phi_{1}+\rho_{1}\left(<v, u>_{\mathcal{N}_{2}}\right) .
$$

$$
\lambda_{i}(w)=\left(\begin{array}{ll}
\psi_{1}^{\prime} & \psi_{2}^{\prime} \\
\phi_{1}^{\prime} & \phi_{2}^{\prime}
\end{array}\right) .
$$

Pour satisfaire à la condition (i) de la définition 4,2, il faut considérer la famille plus large $\left(\lambda_{i}^{g}\right)_{(i, g) \in I \times G}$, où pour tout $(i, g) \in I \times G, i=\left(M_{0}, N_{0}, \epsilon_{0}\right)$,

$$
\begin{array}{rlc}
\lambda_{i}^{g}: g W\left(M_{0}\right) & \longrightarrow & W_{0}^{\prime} \\
x & \longmapsto \lambda_{i}\left(g^{-1} x\right)
\end{array}
$$

On va vérifier les propriétés (ii) et (iii) de la définition 4, 2-, pour montrer que la famille $\left(\lambda_{i}^{g}\right)$ définit un quasi-morphisme fort de $W_{0}$ vers $W_{0}^{\prime}$. On montrera ensuite que c'est un quasiisomorphisme fort. 
Étape 3 - Compatibilité avec l'action des sous-groupes laissant $W\left(M_{0}\right)$ invariant

On considère le sous-groupe $G_{0}$ de $G$ dont la composante dans $\mathbf{B}$ est l'identité. Alors il est clair que pour tout $i=\left(M_{0}, N_{0}, \epsilon_{0}\right) \in I, G_{0}$ laisse invariant l'ouvert $W\left(M_{0}\right)$ de $W_{0}$. On va montrer qu'il existe un morphisme

$$
\gamma_{i}: G_{0} \times W\left(M_{0}\right) \longrightarrow G^{\prime}
$$

tel que pour tous $(g, x) \in G_{0} \times W\left(M_{0}\right)$ on ait

$$
\lambda_{i}(g x)=\gamma_{i}(g, x) \lambda_{i}(x) .
$$

On a un isomorphisme de variétés

$$
\mathbf{R} \times \mathcal{A}_{0} \times G L(M) \times \mathbf{L} \times\left(M^{*} \otimes \mathcal{B}_{0}\right) \simeq G_{0}
$$

qui à $\left(\mathbf{r}, \boldsymbol{\alpha}_{0}, g_{M}, \boldsymbol{\ell}, \beta\right)$ associe le produit des éléments $\left(\begin{array}{cc}\mathbf{r} & 0 \\ 0 & 1\end{array}\right),\left(\begin{array}{cc}1 & 0 \\ \boldsymbol{\alpha}_{0} & 1\end{array}\right),\left(\begin{array}{cc}g_{M} & 0 \\ 0 & 1\end{array}\right)$, $\left(\begin{array}{ll}1 & 0 \\ 0 & \boldsymbol{\ell}\end{array}\right)$ et $\left(\begin{array}{ll}1 & 0 \\ \beta & 1\end{array}\right)$. Il suffit donc de construire pour tout sous-groupe $K$ parmi $\mathbf{R}^{o p}, \mathcal{A}_{0}$, $G L(M)^{o p}, \mathbf{L}, M^{*} \otimes \mathcal{B}_{0}$ un morphisme

$$
\gamma_{K}: K \times W\left(M_{0}\right) \longrightarrow G^{\prime}
$$

tel que pour tous $(g, x) \in K \times W\left(M_{0}\right)$ on ait

$$
\lambda_{i}(g x)=\gamma_{K}(g, x) \lambda_{i}(x) .
$$

On peut éliminer déjà le cas de $G L(M)^{o p}$, car $\lambda_{i}$ est $G L(M)^{o p}$-invariant (on prend $\left.\gamma_{G L(M)^{o p}}=1\right)$. Si $w \in W\left(M_{0}\right)$, on notera $u$, $v$ les éléments de $\mathcal{B}_{0} \otimes \mathcal{N}_{2}, v \in \mathcal{B}_{0}^{\prime} \otimes \mathcal{N}_{2}^{\prime}$ respectivement servant à définir $\lambda_{i}(w)$ (cf. étape 1 ), et $u_{0}, v_{0}$ les éléments analogues servant à définir $\lambda_{i}(g w)$.

On ne donnera que les définitions des morphismes $\gamma_{K}$, les vérifications étant laissées au lecteur.

Définition de $\gamma_{\mathbf{R}^{o p}}$ - Soit $\mathbf{r} \in \mathbf{R}^{o p}$. On prend

$$
\gamma_{\mathbf{R}^{o p}}(\mathbf{r}, w)=\left(I,\left(\begin{array}{cc}
I_{N^{*}} & 0 \\
0 & \mathbf{r}
\end{array}\right)\right) .
$$

Définition de $\gamma_{\mathcal{A}_{0}}$ - Soit $\boldsymbol{\alpha}_{0} \in \mathcal{A}_{0}$. On prend

$$
\gamma_{\mathcal{A}_{0}}\left(\boldsymbol{\alpha}_{0}, w\right)=\left(I,\left(\begin{array}{cc}
1 & 0 \\
\beta^{\prime}\left(\boldsymbol{\alpha}_{0}\right) & 1
\end{array}\right)\right)
$$

l'élément $\beta^{\prime}\left(\boldsymbol{\alpha}_{0}\right)$ de $N \otimes \mathcal{N}_{1}$ étant défini par :

$$
\beta^{\prime}\left(\boldsymbol{\alpha}_{0}\right)(\lambda)=-\nu\left(\boldsymbol{\alpha}_{0} \otimes{ }^{t} s_{M_{0}}\left(\psi_{2}\right)\left({ }^{t} q(\lambda)\right)\right)
$$

pour tout $\lambda \in N^{*}$ (q est défini à la fin de l'étape 1$)$.

Définition de $\gamma_{\mathbf{L}}$ - Soit $\boldsymbol{\ell} \in \mathbf{L}$. On a

$$
\left(I,\left(\begin{array}{ll}
1 & 0 \\
0 & \boldsymbol{\ell}
\end{array}\right)\right) \cdot\left(\begin{array}{ll}
\psi_{1} & \psi_{2} \\
\phi_{1} & \phi_{2}
\end{array}\right)=\left(\begin{array}{cc}
\psi_{1} & \psi_{2} \\
\boldsymbol{\ell} \phi_{1} & \boldsymbol{\ell} \phi_{2}
\end{array}\right) .
$$


Il en découle que $v_{0}=v$ et $u_{0}=-r_{2}\left(\boldsymbol{\ell} \phi_{2}\right)$. On a donc $\rho_{2}\left(u_{0}-\boldsymbol{\ell} u\right)=0$, ce qui entraîne que $u_{0}-\ell u=\overline{\nu^{\prime}}\left(\boldsymbol{\alpha}_{0}^{\prime}\right)$, pour un $\boldsymbol{\alpha}_{0}^{\prime} \in \mathcal{A}_{0}^{\prime}$ uniquement déterminé. On prend alors

$$
\gamma_{\mathcal{A}_{0}}\left(\boldsymbol{\alpha}_{0}, w\right)=\left(\left(\begin{array}{cc}
\boldsymbol{\ell} & 0 \\
\boldsymbol{\alpha}_{0}^{\prime} & 1
\end{array}\right), I\right)
$$

Définition de $\gamma_{M^{*} \otimes \mathcal{B}_{0}}$ - Soit $\beta \in M^{*} \otimes \mathcal{B}_{0}$. On prend

$$
\gamma_{M^{*} \otimes \mathcal{B}_{0}}(\beta, w)=\left(\left(\begin{array}{cc}
1 & 0 \\
\boldsymbol{\alpha}_{0}^{\prime} & 1
\end{array}\right), I\right)
$$

où l'élément $\boldsymbol{\alpha}_{0}^{\prime}$ de $\mathcal{A}_{0}^{\prime}$ est défini comme suit : on a

$$
\left(I,\left(\begin{array}{ll}
1 & 0 \\
\beta & 1
\end{array}\right)\right) \cdot\left(\begin{array}{cc}
\psi_{1} & \psi_{2} \\
\phi_{1} & \phi_{2}
\end{array}\right)=\left(\begin{array}{cc}
\psi_{1} & \psi_{2} \\
\phi_{1}+\rho_{1}\left(<\beta, \psi_{1}>_{M}\right) & \phi_{2}+\rho_{2}\left(<\beta, \psi_{2}>_{M}\right)
\end{array}\right) .
$$

Il en découle que $u_{0}=u-r_{2} \circ \rho_{2}\left(<\beta, \psi_{2}>_{M}\right)$, donc $u_{0}$ s'écrit $u_{0}=u-<\beta, \psi_{2}>_{M}+\overline{\nu^{\prime}}\left(\boldsymbol{\alpha}_{0}^{\prime}\right)$, pour un $\boldsymbol{\alpha}_{0}^{\prime} \in \mathcal{A}_{0}^{\prime}$ uniquement déterminé.

\section{Étape 4 - Compatibilité avec l'action de $\mathbf{B}$}

Soit $\mathbf{b} \in \mathbf{B}$. Soient $i_{0}=\left(M_{0}, N_{0}, \epsilon_{0}\right), i_{1}=\left(M_{1}, N_{1}, \epsilon_{1}\right) \in I$. Alors, si $w=\left(\begin{array}{cc}\psi_{1} & \psi_{2} \\ \phi_{1} & \phi_{2}\end{array}\right) \in W_{0}$, on a $w \in W\left(M_{0}\right) \cap \mathbf{b}^{-1} W\left(M_{1}\right)$ si et seulement si $\operatorname{ker}\left(\overline{\psi_{2}}\right) \cap M_{0}=\operatorname{ker}\left(\overline{\psi_{2}}\right) \cap \mathbf{b}\left(M_{1}\right)=\{0\}$. Soit $U\left(M_{0}, M_{1}\right)$ l'ouvert de $W_{0} \times \mathbf{B}$ constitué des points $(w, \mathbf{b})$ tels que $w \in W\left(M_{0}\right) \cap \mathbf{b}^{-1} W\left(M_{1}\right)$. On montre comme dans l'Étape 3 qu'il existe un morphisme

$$
\delta_{i_{0} i_{1}}: U\left(M_{0}, M_{1}\right) \longrightarrow G^{\prime}
$$

tel que pour tout $(w, \mathbf{b}) \in U\left(M_{0}, M_{1}\right)$ on ait

$$
\lambda_{i_{1}}(\mathbf{b} w)=\delta_{i_{0} i_{1}}(\mathbf{b}, w) \lambda_{i_{0}}(w) .
$$

Étape 5 - Vérification des propriétés (i) et (ii) de la définition 4

Le fait que la famille $\left(\lambda_{i}^{g}\right)_{(i, g) \in I \times G}$ définie dans l'étape 2 vérifie les propriétés (i) et (ii) de la définition 4 découle immédiatement des étapes 3 et 4 . On a donc défini un quasi-morphisme fort de $W_{0}$ vers $W_{0}^{\prime}$ au dessus de $D_{\theta}$.

Il reste à montrer que c'est un quasi-isomorphisme fort. Pour cela on définit la famille $\left(\lambda_{j}^{g^{\prime}}\right)_{(j, g) \in J \times G^{\prime}}$, où $J$ est l'ensemble des triplets $\left(M_{0}^{\prime}, N_{0}^{\prime}, \epsilon_{0}^{\prime}\right)$, où $N_{0}^{\prime} \oplus M_{0}^{\prime}$ est une décomposition en somme directe de $\mathcal{N}_{2}$ avec $\operatorname{dim}\left(N_{0}^{\prime}\right)=\operatorname{dim}(N)$, et $\epsilon_{0}^{\prime}: M^{*} \longrightarrow M_{0}^{\prime}$ un isomorphisme. Pour tout $j=\left(M_{0}^{\prime}, N_{0}^{\prime}, \epsilon_{0}^{\prime}\right) \in J, \lambda_{j}^{g^{\prime}}$ est un morphisme de $g^{\prime} U^{\prime}\left(N_{0}^{\prime}\right)$ dans $W_{0}$ au dessus de $D_{D(\theta)}$, $U^{\prime}\left(N_{0}^{\prime}\right)$ étant l'ouvert de $W_{0}^{\prime}$ constitué des éléments $\left(\begin{array}{cc}\psi_{1}^{\prime} & \psi_{2}^{\prime} \\ \phi_{1}^{\prime} & \phi_{2}^{\prime}\end{array}\right)$ tels que $\operatorname{ker}\left(\overline{\psi_{2}^{\prime}}\right) \cap N_{0}^{\prime}=\{0\}$. La définition des $\lambda_{j}^{g^{\prime}}$ est semblable à celle des $\lambda_{i}^{g}$ de l'étape 2. 
Étape 6 - $\left(\lambda_{i}^{g}\right)_{(i, g) \in I \times G}$ est un quasi-isomorphisme fort

Soient $(i, g) \in I \times G$ et $\left(j, g^{\prime}\right) \in J \times G^{\prime}$, avec $j=\left(N_{0}^{\prime}, M_{0}^{\prime}, \epsilon_{0}^{\prime}\right)$. Il suffit de montrer que

$$
\phi=\lambda_{j}^{g^{\prime}} \circ \lambda_{i}^{g}: V=\left(\lambda_{i}^{g}\right)^{-1}\left(g^{\prime} U^{\prime}\left(N_{0}^{\prime}\right)\right) \longrightarrow W_{0}
$$

est une carte de $I_{W_{0}}$, c'est-à-dire qu'il existe un morphisme $\gamma: V \longrightarrow G$ tel que pour tout $w \in V$ on ait $\phi(x)=\gamma(x) . x$. Cela se démontre comme dans les étapes 3 à 5 et est laissé au lecteur.

\subsubsection{Correspondance entre les quotients}

On déduit du théorème 4.11 et de la proposition 4.8 le

Théorème 4.12. Soient $U$ un ouvert $G$-invariant de $W_{0}^{\prime}$ tel qu'il existe un quasi-bon quotient $U / / G$ et $U^{\prime}$ l'ouvert correspondant de $W_{0}^{\prime}$. Alors il existe un quasi-bon quotient $U^{\prime} / / G^{\prime}$ canoniquement isomorphe à $U / / G$.

Pour obtenir des bons quotients il faut faire des hypothèses supplémentaires, pour appliquer les propositions 4.9 ou 4.10 . C'est ce qu'on va faire au chapitre suivant pour étudier les morphismes de type $(r, s)$.

\section{LE CAS DES MORPhismes DE TYPE $(r, s)$}

\subsection{Introduction}

On applique les résultats du chapitre 4 aux cas décrits au $\S 3.3$.

\subsubsection{Mutations des morphismes de type $(r, s)$}

Soient $X$ une variété projective, $r, s$ des entiers positifs, et $\mathcal{E}_{1}, \ldots, \mathcal{E}_{r},, \mathcal{F}_{1}, \ldots, \mathcal{F}_{s}$ des faisceaux cohérents simples sur $X$ tels que

$$
\begin{gathered}
\operatorname{Hom}\left(\mathcal{E}_{i}, \mathcal{E}_{i^{\prime}}\right)=0 \text { si } i>i^{\prime}, \operatorname{Hom}\left(\mathcal{F}_{j}, \mathcal{F}_{j^{\prime}}\right)=0 \text { si } j>j^{\prime}, \\
\operatorname{Hom}\left(\mathcal{F}_{j}, \mathcal{E}_{i}\right)=\{0\} \text { pour tous } i, j .
\end{gathered}
$$


On suppose que pour $1 \leq i \leq r$ le morphisme canonique

$$
\mathcal{E}_{i} \longrightarrow \operatorname{Hom}\left(\mathcal{E}_{i}, \mathcal{F}_{1}\right)^{*} \otimes \mathcal{F}_{1}
$$

est injectif. Soit $\mathcal{G}_{i}$ son conoyau. On suppose que

$$
\operatorname{Ext}^{1}\left(\mathcal{F}_{1}, \mathcal{F}_{j}\right)=\operatorname{Ext}^{1}\left(\mathcal{F}_{1}, \mathcal{E}_{i}\right)=\operatorname{Ext}^{1}\left(\mathcal{E}_{i}, \mathcal{F}_{1}\right)=\operatorname{Ext}^{1}\left(\mathcal{E}_{i}, \mathcal{E}_{k}\right)=\operatorname{Ext}^{1}\left(\mathcal{G}_{i}, \mathcal{F}_{j}\right)=\{0\}
$$

si $1 \leq i \leq k \leq r, 2 \leq j \leq s$. Soient $M_{1}, \ldots, M_{r}, N_{1}, \ldots, N_{s}$ des $\mathbb{C}$-espaces vectoriels de dimension finie $m_{1}, \ldots, m_{r}, n_{1}, \ldots, n_{s}$ respectivement. On s'intéresse aux morphismes

$$
\bigoplus_{1 \leq i \leq r}\left(\mathcal{E}_{i} \otimes M_{i}\right) \longrightarrow \bigoplus_{1 \leq l \leq s}\left(\mathcal{F}_{l} \otimes N_{l}\right)
$$

Soit $p$ un entier tel que $0 \leq p \leq r-1$. On pose (cf. 3.2 )

$$
\begin{array}{cc}
\mathcal{U}_{1}=\bigoplus_{1 \leq i \leq p}\left(\mathcal{E}_{i} \otimes M_{i}\right), \quad \mathcal{U}_{2}=\bigoplus_{p+1 \leq j \leq r}\left(\mathcal{E}_{j} \otimes M_{j}\right), \\
\Gamma=\mathcal{F}_{1}, M=N_{1}, \quad \mathcal{V}_{1}=\bigoplus_{2 \leq l \leq s}\left(\mathcal{F}_{l} \otimes N_{l}\right) .
\end{array}
$$

de sorte que les morphismes précédents peuvent s'écrire sous la forme

$$
\mathcal{U}_{1} \oplus \mathcal{U}_{2} \longrightarrow(\Gamma \otimes M) \oplus \mathcal{V}_{1},
$$

comme dans le chapitre 4 . On suppose que $\operatorname{dim}(M)<\operatorname{dim}\left(\operatorname{Hom}\left(\mathcal{U}_{2}, \Gamma\right)\right)$. Soit $N$ un $\mathbb{C}$-espace vectoriel tel que $\operatorname{dim}(M)+\operatorname{dim}(N)=\operatorname{dim}\left(\operatorname{Hom}\left(\mathcal{U}_{2}, \Gamma\right)\right)$. On s'intéresse maintenant aux mutations des morphismes précédents. Ce sont des morphismes du type

$$
\mathcal{U}_{1} \oplus(\Gamma \otimes N) \longrightarrow \mathcal{V}_{2} \oplus \mathcal{V}_{1}
$$

c'est-à-dire

$$
\left(\bigoplus_{1 \leq i \leq p}\left(\mathcal{E}_{i} \otimes M_{i}\right)\right) \oplus\left(\mathcal{F}_{1} \otimes N\right) \longrightarrow\left(\bigoplus_{p<j \leq r}\left(\mathcal{G}_{j} \otimes M_{j}\right)\right) \oplus\left(\bigoplus_{2 \leq l \leq s}\left(\mathcal{F}_{l} \otimes N_{l}\right)\right) .
$$

Remarque : Les morphismes de départ ne dépendent pas de $p$, mais les mutations de morphismes vont en dépendre.

Définition de l'espace abstrait de morphismes $\Theta_{p}$

Il correspond aux morphismes $\mathcal{U}_{1} \oplus \mathcal{U}_{2} \longrightarrow(\Gamma \otimes M) \oplus \mathcal{V}_{1}$. On pose

$$
H_{l i}=\operatorname{Hom}\left(\mathcal{E}_{i}, \mathcal{F}_{l}\right), \quad A_{j i}=\operatorname{Hom}\left(\mathcal{E}_{i}, \mathcal{E}_{j}\right), \quad B_{m l}=\operatorname{Hom}\left(\mathcal{F}_{l}, \mathcal{F}_{m}\right) .
$$

On a $n_{1}=\operatorname{dim}\left(N_{1}\right)<\sum_{1 \leq i \leq r} \operatorname{dim}\left(H_{1 i}\right) m_{i}$. On pose

$$
\begin{gathered}
\mathcal{N}_{1}=\bigoplus_{1 \leq i \leq p}\left(H_{1 i} \otimes M_{i}^{*}\right)=\operatorname{Hom}\left(\mathcal{U}_{1}, \Gamma\right), \quad \mathcal{N}_{2}=\bigoplus_{p+1 \leq j \leq r}\left(H_{1 j} \otimes M_{j}^{*}\right)=\operatorname{Hom}\left(\mathcal{U}_{2}, \Gamma\right) \\
\mathcal{M}_{1}=\bigoplus_{1 \leq i \leq p, 2 \leq l \leq s}\left(H_{l i} \otimes M_{i}^{*} \otimes N_{l}\right)=\operatorname{Hom}\left(\mathcal{U}_{1}, \mathcal{V}_{1}\right)
\end{gathered}
$$




$$
\begin{gathered}
\mathcal{M}_{2}=\bigoplus_{p+1 \leq j \leq r, 2 \leq l \leq s}\left(H_{l j} \otimes M_{j}^{*} \otimes N_{l}\right)=\operatorname{Hom}\left(\mathcal{U}_{2}, \mathcal{V}_{1}\right), \\
\mathcal{A}_{0}=\bigoplus_{1 \leq i \leq p, p+1 \leq j \leq r}\left(A_{j i} \otimes M_{i}^{*} \otimes M_{j}\right)=\operatorname{Hom}\left(\mathcal{U}_{1}, \mathcal{U}_{2}\right), \\
M=N_{1}, \quad \mathcal{B}_{0}=\bigoplus_{2 \leq l \leq s}\left(B_{l 1} \otimes N_{l}\right)=\operatorname{Hom}\left(\Gamma, \mathcal{V}_{1}\right) .
\end{gathered}
$$

On définit de même les groupes $\mathbf{L}, \mathbf{R}, \mathbf{B}$, et les applications $\nu, \mu, \rho_{1}$, et $\rho_{2}$. On obtient ainsi un espace abstrait de morphismes noté $\Theta_{p}$, d'espace total de morphismes noté $W_{p}$. On a

$$
W_{p}=W=\bigoplus_{1 \leq i \leq r, 1 \leq s \leq l}\left(H_{l i} \otimes M_{i}^{*} \otimes N_{l}\right),
$$

mais en général $W_{p}^{0} \neq W_{q}^{0}$ si $p \neq q$.

Soit

$$
w=\left(\psi_{l i}\right)_{1 \leq i \leq r, 1 \leq s \leq s} \in W .
$$

Alors, par définition, on a $w \in W_{p}^{0}$ si et seulement si l'application linéaire

$$
\sum_{p+1 \leq j \leq r} \psi_{j}: \bigoplus_{p+1 \leq j \leq r}\left(H_{1 j}^{*} \otimes M_{j}\right) \longrightarrow N_{1}
$$

déduite de $w$ est surjective. On a donc $W_{r-1}^{0} \subset W_{r-2}^{0} \subset \cdots \subset W_{0}^{0} \subset W$.

\subsubsection{Plan de la suite du chapitre 5}

Dans les $\$ 5.2$ et 5.3 , on donne la description de l'espace abstrait de morphismes $D\left(\Theta_{p}\right)$. On note $W^{\prime}(p)$ son espace total, et $G(p)$ le groupe qui agit sur $W^{\prime}(p)$. L'espace abstrait $D\left(\Theta_{p}\right)$ correspond en fait à un espace de morphismes de type $(r+s-p-1, p+1)$ (les morphismes obtenus dans le théorème 3.3.

On rappelle au $\S 5.4$ des résultats de [6] permettant de construire des bons quotients de certains ouverts $G$-invariants de $W$. On obtient une notion de (semi-)stabilité sous l'action de $G$ en fixant une suite de nombre rationnels, appelée polarisation : $\Lambda=\left(\lambda_{1}, \ldots, \lambda_{r}, \mu_{1}, \ldots, \mu_{s}\right)$. On définit au $\$ 5.5$ une polarisation $\Lambda_{p}^{\prime}$ de l'action de $G(p)$ sur $W^{\prime}(p)$ naturellement associée à la précédente.

Dans le $\$ 5.6$ on donne des conditions pour qu'il y ait équivalence entre la (semi-)stabilité d'un morphisme de $W$ relativement à $\Lambda$ et la semi-stabilité relativement à $\Lambda_{p}^{\prime}$ des mutations de ce morphisme. On en déduit, à l'aide des théorème 4.11, 4.12 et des résultats du $\S 4.4 .3$ les théorèmes 5.6, 5.7, 5.8 et 5.9, ce dernier étant une amélioration du théorème 5.1 obtenu dans [6]. On peut ainsi construire des variétés de modules de morphismes pour d'autres polarisations que celles qui sont indiquées dans [6]. De nombreux exemples sont donnés au chapitre 6 . 


\subsection{Description des mutations d'espaces de morphismes abstraits}

L'espace de morphismes abstraits $D\left(\Theta_{p}\right)$ correspond à un espace de morphismes de type $(p+1, r+s-p-1)$. Rappelons qu'il s'agit des morphismes

$$
\mathcal{U}_{1} \oplus(\Gamma \otimes N) \longrightarrow \mathcal{V}_{2} \oplus \mathcal{V}_{1}
$$

c'est-à-dire

$$
\left(\bigoplus_{1 \leq i \leq p}\left(\mathcal{E}_{i} \otimes M_{i}\right)\right) \oplus\left(\mathcal{F}_{1} \otimes N\right) \longrightarrow\left(\bigoplus_{p<j \leq r}\left(\mathcal{G}_{j} \otimes M_{j}\right)\right) \oplus\left(\bigoplus_{2 \leq l \leq s}\left(\mathcal{F}_{l} \otimes N_{l}\right)\right) .
$$

On les notera plutôt

$$
\bigoplus_{1 \leq i \leq p+1}\left(\mathcal{E}_{i}^{\prime} \otimes M_{i}^{(p)}\right) \longrightarrow \bigoplus_{1 \leq l \leq r+s-p-1}\left(\mathcal{F}_{l}^{\prime} \otimes N_{l}^{(p)}\right),
$$

par analogie avec les morphismes de départ.

On a donc

$$
\begin{gathered}
\mathcal{E}_{i}^{\prime}=\mathcal{E}_{i} \text { si } 1 \leq i \leq p, \quad \mathcal{E}_{p+1}^{\prime}=\mathcal{F}_{1}, \\
\mathcal{F}_{j}^{\prime}=\mathcal{G}_{j+p} \quad \text { si } 1 \leq j \leq r-p, \quad \mathcal{F}_{j}^{\prime}=\mathcal{F}_{j-r+p+1} \quad \text { si } r-p+1 \leq j \leq r+s-p-1, \\
M_{i}^{(p)}=M_{i} \text { si } 1 \leq i \leq p, \\
\operatorname{dim}\left(M_{p+1}^{(p)}\right)=\left(\sum_{p+1 \leq j \leq r} m_{j} \operatorname{dim}\left(H_{1 j}\right)\right)-n_{1}, \\
N_{i}^{(p)}=M_{p+i} \text { si } 1 \leq i \leq r-p, \quad N_{l}^{(p)}=N_{l-r+p+1} \quad \text { si } r-p+1 \leq l \leq r+s-p-1,
\end{gathered}
$$

On pose

$$
H_{l i}^{(p)}=\operatorname{Hom}\left(\mathcal{E}_{i}^{\prime}, \mathcal{F}_{l}^{\prime}\right), \quad A_{j i}^{(p)}=\operatorname{Hom}\left(\mathcal{E}_{i}^{\prime}, \mathcal{E}_{j}^{\prime}\right), \quad B_{m l}^{(p)}=\operatorname{Hom}\left(\mathcal{F}_{l}^{\prime}, \mathcal{F}_{m}^{\prime}\right) .
$$

On a donc

$$
\begin{gathered}
A_{j i}^{(p)}=A_{j i} \text { si } 1 \leq i \leq j \leq p, \quad A_{p+1, i}^{(p)}=H_{1 i} \quad \text { si } 1 \leq i \leq p, \\
B_{l m}^{(p)}=A_{l+p, m+p} \text { si } 1 \leq m \leq l \leq r-p, \\
B_{l m}^{(p)}=B_{l-r+p+1, m-r+p+1} \quad \text { si } r-p+1 \leq m \leq l \leq r+s-p-1, \\
B_{l m}^{(p)}=\operatorname{ker}\left(B_{l-r+p+1,1} \otimes H_{1, m+p} \longrightarrow H_{l-r+p+1, m+p}\right) \\
\quad \sin r+p+1 \leq l \leq r+s-p-1,1 \leq m \leq r-p, \\
H_{l i}^{(p)}=\left(H_{1 i} \otimes H_{1, l+p}^{*}\right) / A_{l+p, i} \quad \text { si } 1 \leq i \leq p, 1 \leq l \leq r-p, \\
H_{l i}^{(p)}=H_{l-r+p+1, i} \text { si } 1 \leq i \leq p, r-p+1 \leq l \leq r+s-p-1, \\
H_{l, p+1}^{(p)}=H_{1, l+p}^{*} \text { si } 1 \leq l \leq r-p, \quad H_{l, p+1}^{(p)}=B_{l-r+p+1,1} \quad \text { si } r-p+1 \leq l \leq r+s-p-1 .
\end{gathered}
$$

La description des compositions est laissée au lecteur. On note

$$
W^{\prime}(p)=\bigoplus_{1 \leq i \leq p+1,1 \leq l \leq r+s-p-1} L\left(H_{l i}^{(p) *} \otimes M_{i}^{(p)}, N_{l}^{(p)}\right)
$$

l'espace total de morphismes de $D\left(\Theta_{p}\right), W_{0}^{\prime}(p)$ l'ouvert correspondant, $G(p)$ le groupe agissant sur $W^{\prime}(p)$. Le théorème 4.7 dit qu'on a une bijection

$$
W_{p}^{0} / G \simeq W_{0}^{\prime}(p) / G(p) .
$$


Rappelons que $W_{0}^{\prime}(p)$ est l'ouvert des morphismes de type $(*)$ précédent tels que l'application linéaire associée

$$
N=M_{p+1}^{(p)} \longrightarrow \bigoplus_{1 \leq j \leq r-p} H_{j, p+1}^{(p)} \otimes N_{j, p+1}^{(p)}
$$

soit injective.

\subsection{Description des mutations de morphismes}

Cette description n'est utilisée que dans la démonstration de la proposition 5.2 .

Soit

$$
w=\left(\theta_{l i}\right)_{1 \leq i \leq r, 1 \leq l \leq s} \in W_{p}^{0} \subset \bigoplus_{1 \leq i \leq r, 1 \leq l \leq s} L\left(H_{l i}^{*} \otimes M_{i}, N_{l}\right),
$$

avec $\theta_{l i} \in L\left(H_{l i}^{*} \otimes M_{i}, N_{l}\right)=\operatorname{Hom}\left(\mathcal{E}_{i} \otimes M_{i}, \mathcal{F}_{l} \otimes N_{l}\right)$, et

$$
\begin{array}{ll}
\psi_{1}=\left(\theta_{1 i}\right)_{1 \leq i \leq p} \in \operatorname{Hom}\left(\mathcal{U}_{1}, \Gamma \otimes M\right), & \psi_{2}=\left(\theta_{1 j}\right)_{p+1 \leq j \leq r} \in \operatorname{Hom}\left(\mathcal{U}_{2}, \Gamma \otimes M\right), \\
\phi_{1}=\left(\theta_{l i}\right)_{1 \leq i \leq p, 2 \leq l \leq s} \in \operatorname{Hom}\left(\mathcal{U}_{1}, \mathcal{V}_{1}\right), & \phi_{2}=\left(\theta_{l i}\right)_{p+1 \leq i \leq r, 2 \leq l \leq s} \in \operatorname{Hom}\left(\mathcal{U}_{2}, \mathcal{V}_{1}\right) .
\end{array}
$$

On va décrire

$$
z(w)=\left(\begin{array}{cc}
\psi_{1}^{\prime} & \psi_{2}^{\prime} \\
\phi_{1}^{\prime} & \phi_{2}^{\prime}
\end{array}\right)
$$

(les notations sont en accord avec celles du $\S 3.2 .2$ et du chapitre 4). On construit d'abord les éléments $u$ et $v$ des $\S 3.2 .2$ et 4.3 . On doit prendre pour $u$ un élément de

$$
\mathcal{B}_{0} \otimes \mathcal{N}_{2}=\bigoplus_{p+1 \leq j \leq r, 2 \leq l \leq s}\left(H_{1 j} \otimes B_{l 1} \otimes M_{j}^{*} \otimes N_{l}\right)
$$

tel que le diagramme suivant soit commutatif :

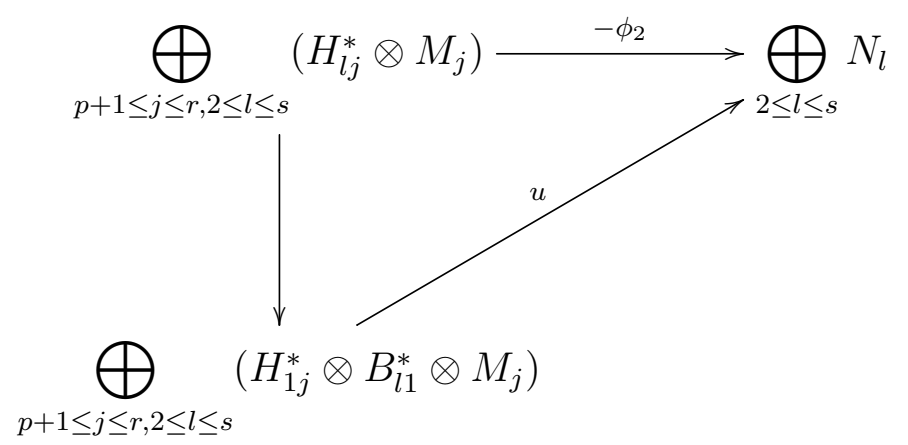

La flèche verticale est induite par les compositions $B_{l 1} \otimes H_{1 j} \longrightarrow H_{l j}$. Le diagramme précédent traduit le fait qu'on doit avoir $\rho_{2}(u)=-\phi_{2} \quad(\mathrm{cf} . \S 4.3)$.

On prend pour $v$ un élément de

$$
\mathcal{N}_{1} \otimes \mathcal{N}_{2}^{*}=\bigoplus_{1 \leq i \leq p, p+1 \leq j \leq r}\left(H_{1 j}^{*} \otimes H_{1 i} \otimes M_{i}^{*} \otimes M_{j}\right)
$$

tel que le diagramme suivant soit commutatif : 


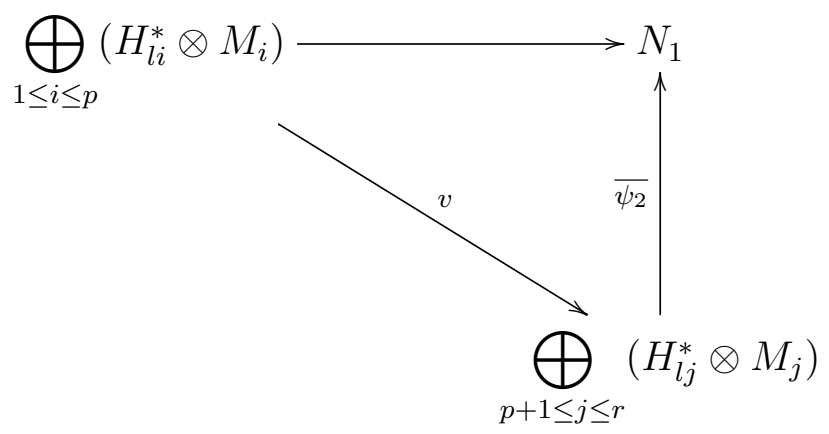

La flèche horizontale provient de $\psi_{1}$. Le diagramme précédent traduit le fait que $\left(I_{\mathcal{N}_{1}} \otimes \overline{\psi_{2}}\right)(v)=\psi_{1} \quad($ cf. $\S 4.3$.

Déterminons maintenant $\psi_{1}^{\prime}, \psi_{2}^{\prime}, \phi_{1}^{\prime}$ et $\phi_{2}^{\prime}$. On fixe un isomorphisme $N \simeq \operatorname{ker}\left(\overline{\psi_{2}}\right)$, et $N$ s'identifie donc à un quotient de $\bigoplus_{p+1 \leq j \leq r}\left(H_{l j}^{*} \otimes M_{j}\right)$. Alors $\phi_{2}^{\prime}$ est l'image de $v$ dans

$$
\bigoplus_{1 \leq i \leq p, p+1 \leq j \leq r}\left(\left(\left(H_{1 j}^{*} \otimes H_{1 i}\right) / A_{j i}\right) \otimes M_{i}^{*} \otimes M_{j}\right)=\bigoplus_{1 \leq i \leq p, 1 \leq l \leq r-p}\left(H_{l i}^{(p)} \otimes M_{i}^{(p) *} \otimes N_{l}^{(p)}\right),
$$

(cela traduit le fait que $\phi_{2}^{\prime}=\rho_{2}^{\prime}(v)$, cf. $\left.\S 4.3\right), \psi_{2}^{\prime}$ est l'inclusion

$$
\operatorname{ker}\left(\overline{\psi_{2}}\right) \subset \bigoplus_{p+1 \leq j \leq r}\left(H_{l j}^{*} \otimes M_{j}\right)
$$

et $\psi_{1}^{\prime}$ est la restriction à $\operatorname{ker}\left(\overline{\psi_{2}}\right)$ de l'application linéaire

$$
\bigoplus_{p+1 \leq j \leq r}\left(H_{1 j}^{*} \otimes M_{j}\right) \longrightarrow \bigoplus_{2 \leq l \leq s}\left(B_{l 1} \otimes N_{l}\right)
$$

provenant de $u$ (cela traduit le fait que $\psi_{1}^{\prime}=\left(I_{\mathcal{N}_{1}} \otimes \overline{\psi_{2}^{\prime}}\right)(u)$, cf. $\S 4.3$. Pour obtenir $\phi_{1}^{\prime}$, on fait la somme de $\phi_{1}$ et de la composée

$$
\bigoplus_{1 \leq i \leq r}\left(H_{1 i}^{*} \otimes M_{i}\right) \stackrel{v}{\longrightarrow} \bigoplus_{p+1 \leq j \leq r}\left(H_{1 j}^{*} \otimes M_{j}\right) \stackrel{u}{\longrightarrow} \bigoplus_{2 \leq l \leq s}\left(B_{l 1} \otimes N_{l}\right)
$$

(cela traduit le fait que $\phi_{1}^{\prime}=\phi_{1}+\rho_{1}\left(<v, u>_{\mathcal{N}_{2}}\right)$ ).

\subsection{Construction des variétés de modules de morphismes de type $(r, s)$}

Les résultats de cette section provienne de [6].

\subsubsection{Notions de (semi-)stabilité}

On veut définir une notion de (semi-)stabilité pour les points de

$$
W=\operatorname{Hom}\left(\bigoplus_{1 \leq i \leq r}\left(\mathcal{E}_{i} \otimes M_{i}\right), \bigoplus_{1 \leq l \leq s}\left(\mathcal{F}_{l} \otimes N_{l}\right)\right)
$$


On identifie les éléments de $G_{L}$ à des matrices

$$
\left(\begin{array}{cccccc}
g_{1} & 0 & . & . & . & 0 \\
u_{21} & g_{2} & \cdot & . & . & 0 \\
\cdot & \cdot & \cdot & & & \cdot \\
\cdot & & & \cdot & & \cdot \\
\cdot & & u_{i j} & & \cdot & \cdot \\
u_{r 1} & . & . & . & . & g_{r}
\end{array}\right)
$$

avec $g_{i} \in G L\left(M_{i}\right)$ et $u_{i j} \in \operatorname{Hom}\left(\mathcal{E}_{j} \otimes M_{j}, \mathcal{E}_{i} \otimes M_{i}\right)$, et on a une représentation analogue des éléments de $G_{R}$.

On ne peut pas appliquer la géométrie invariante à l'action de $G$ sur $W$ si $r>1$ ou $s>1$ car le groupe $G$ n'est pas réductif. On va définir deux sous-groupes canoniques de $G$. Soit $H_{L}$ (resp. $G_{L, \text { red }}$ ) le sous-groupe de $G_{L}$ formé des matrices du type précédent telles que $g_{i}=I_{M_{i}}$ pour $1 \leq i \leq r$ (resp. $u_{i j}=0$ pour $1 \leq j<i \leq r$ ). Alors $H_{L}$ est un sous-groupe unipotent normal maximal de $G_{L}, G_{L, \text { red }}$ est un sous-groupe réductif de $G_{L}$ et l'inclusion $G_{L, r e d} \subset G_{L}$ induit un isomorphisme $G_{L, \text { red }} \simeq G_{L} / H_{L}$. On définit de même les sous-groupes $H_{R}$ et $G_{R, r e d}$ de $G_{R}$.

Maintenant soient $H=H_{L} \times H_{R}^{o p}, G_{r e d}=G_{L, r e d} \times G_{R, r e d}^{o p}$. Alors $H$ est un sous-groupe unipotent normal maximal de $G$ et $G_{r e d}$ est un sous-groupe réductif de $G$.

L'action de $G_{r e d}$ sur $W$ est un cas particulier des actions traitées dans [9]. Soient $\lambda_{1}, \ldots, \lambda_{r}$, $\mu_{1}, \ldots, \mu_{s}$ des nombres rationnels positifs tels que

$$
\sum_{1 \leq i \leq r} \lambda_{i} m_{i}=\sum_{1 \leq l \leq s} \mu_{l} n_{l} .
$$

Définition 7. On dit qu'un élément $\left(\theta_{l i}\right)$ de $W$ est $G_{r e d}$-semi-stable (resp. $G_{r e d}$-stable) relativement à $\left(\lambda_{1}, \ldots, \lambda_{r}, \mu_{1}, \ldots, \mu_{s}\right)$ si la propriété suivante est vérifiée : soient $M_{i}^{\prime} \subset M_{i}, N_{l}^{\prime} \subset N_{l}$ des sous-espaces vectoriels tels que l'un au moins des $N_{l}^{\prime}$ soit distinct de $N_{l}$ et que pour $1 \leq i \leq r$, $1 \leq l \leq s$, on ait

Alors on a

$$
\theta_{l i}\left(H_{l i}^{*} \otimes M_{i}^{\prime}\right) \subset N_{l}^{\prime}
$$

$$
\sum_{1 \leq i \leq r} \lambda_{i} \operatorname{dim}\left(M_{i}^{\prime}\right) \leq \sum_{1 \leq l \leq s} \mu_{l} \operatorname{dim}\left(N_{l}^{\prime}\right) \quad(\text { resp. }<)
$$

Définition 8. On dit qu'un élément $x$ de $W$ est $G$-semi-stable ( resp. G-stable) relativement $\grave{a}\left(\lambda_{1}, \ldots, \lambda_{r}, \mu_{1}, \ldots, \mu_{s}\right)$ si tous les points de l'orbite $H . x$ sont $G_{r e d}$-semi-stables ( resp. $G_{r e d}{ }^{-}$ stables) relativement $\grave{a}\left(\lambda_{1}, \ldots, \lambda_{r}, \mu_{1}, \ldots, \mu_{s}\right)$.

On note $W^{s s}\left(\lambda_{1}, \ldots, \lambda_{r}, \mu_{1}, \ldots, \mu_{s}\right)$ (resp. $\left.W^{s}\left(\lambda_{1}, \ldots, \lambda_{r}, \mu_{1}, \ldots, \mu_{s}\right)\right)$, ou plus simplement $W^{s s}$ (resp. $\left.W^{s}\right)$ si aucune confusion n'est à craindre, l'ouvert de $W$ constitué des points $G$-semistables ( resp. $G$-stables) relativement à $\left(\lambda_{1}, \ldots, \lambda_{r}, \mu_{1}, \ldots, \mu_{s}\right)$. 


\subsubsection{Cas d'existence d'un bon quotient projectif}

On donne dans [6] des conditions suffisantes portant sur $\lambda_{1}, \ldots, \lambda_{r}, \mu_{1}, \ldots, \mu_{s}$, pour qu'il existe un bon quotient

$$
\pi: W^{s s} \longrightarrow M=M\left(\lambda_{1}, \ldots, \lambda_{r}, \mu_{1}, \ldots, \mu_{s}\right)
$$

par $G$ avec $M$ projective. Dans ce cas $M$ est normale et la restriction de $\pi$

$$
W^{s} \longrightarrow M^{s}=\pi\left(W^{s}\right)
$$

est un quotient géométrique. Le résultat le plus général est assez compliqué. Rappelons simplement ici le cas des morphismes de type $(2,1)$, le seul qu'on utilisera ici (dans le chapitre 6).

On s'intéresse donc à des morphismes

$$
\left(\mathcal{E}_{1} \otimes M_{1}\right) \oplus\left(\mathcal{E}_{2} \otimes M_{2}\right) \longrightarrow \mathcal{F}_{1} \otimes N_{1}
$$

Il faut d'abord définir certaines constantes. Soit $k>0$ un entier. Soient

$$
\tau: H_{11}^{*} \otimes A_{21} \longrightarrow H_{12}^{*}
$$

l'application linéaire déduite de la composition $H_{12} \otimes A_{21} \longrightarrow H_{11}$, et

$$
\tau_{k}=\tau \otimes I_{\mathbb{C}^{k}}: H_{11}^{*} \otimes\left(A_{21} \otimes \mathbb{C}^{k}\right) \longrightarrow H_{12}^{*} \otimes \mathbb{C}^{k} .
$$

Soit $\mathcal{K}$ l'ensemble des sous-espaces vectoriels propres $K \subset A_{21} \otimes \mathbb{C}^{k}$ tels que pour tout sousespace propre $F \subset \mathbb{C}^{k}, K$ ne soit pas contenu dans $A_{21} \otimes F$. Alors posons

$$
c(\tau, k)=\sup _{K \in \mathcal{K}}\left(\frac{\operatorname{codim}\left(\tau_{k}\left(H_{11}^{*} \otimes K\right)\right.}{\operatorname{codim}(K)}\right) .
$$

Dans le cas des morphismes de type $(2,1)$, les notions de semi-stabilité sont définies à partir de triplets

$$
\left(\lambda_{1}, \lambda_{2}, \frac{1}{n_{1}}\right)
$$

tels que $\lambda_{1} m_{1}+\lambda_{2} m_{2}=1$. Elles dépendent donc essentiellement d'un paramètre. On les exprimera donc en fonction de

$$
t=m_{2} \lambda_{2}
$$

qui est un nombre rationnel dans l'intervalle ]0,1[. Le résultat suivant est démontré dans [6] :

Théorème 5.1. Il existe un bon quotient projectif $W^{s s} / / G$ dès que

$$
t>\frac{m_{2} \operatorname{dim}\left(\operatorname{Hom}\left(\mathcal{E}_{1}, \mathcal{E}_{2}\right)\right)}{m_{2} \operatorname{dim}\left(\operatorname{Hom}\left(\mathcal{E}_{1}, \mathcal{E}_{2}\right)\right)+m_{1}} \quad \text { et } \quad t>\operatorname{dim}\left(\operatorname{Hom}\left(\mathcal{E}_{1}, \mathcal{E}_{2}\right)\right) . c\left(\tau, m_{2}\right) \frac{m_{2}}{n_{1}} .
$$

Remarque : il découle de la construction des quotients $W^{s s} / / G$ dans [6] que si les hypothèses du théorème précédent sont vérifiées, alors il existe un caractère $\chi$ de $G$ dépendant de la polarisation tel que $W^{s s}=W^{s s}(\chi)$ (cf. 4.4.3). De plus il existe un recouvrement de $W^{s s} / / G$ par des ouverts affines dont l'image inverse dans $W^{s s}$ est de la forme $W_{f}, f$ étant un polynôme $\chi^{k}$-invariant (pour un entier $k>0$ ). 


\subsection{Polarisations associées}

Soit $\left(\lambda_{1}, \ldots, \lambda_{r}, \mu_{1}, \ldots, \mu_{s}\right)$ une polarisation de l'action de $G$ sur $W$. On va en déduire une polarisation de l'action de $G(p)$ sur $W^{\prime}(p)$.

Soient $M_{i}^{\prime} \subset M_{i}, N_{l}^{\prime} \subset N_{l}, 1 \leq i \leq r, 1 \leq l \leq s$ des sous-espaces vectoriels. On pose

$$
\begin{gathered}
m_{i}^{\prime}=\operatorname{dim}\left(M_{i}^{\prime}\right), \quad n_{l}^{\prime}=\operatorname{dim}\left(N_{l}^{\prime}\right), \\
\mathbb{M}_{i}=M_{i}^{\prime} \text { si } 1 \leq i \leq p, \\
\mathbb{N}_{l}=M_{l-p}^{\prime} \text { si } 1 \leq l \leq r-p, \quad \mathbb{N}_{l}=N_{l-r+p+1}^{\prime} \text { si } r-p+1 \leq l \leq r+s-p-1, \\
\mathbf{m}_{i}=\operatorname{dim}\left(\mathbb{M}_{i}\right) \quad \text { si } 1 \leq i \leq p, \quad \mathbf{m}_{p+1}=\left(\sum_{p+1 \leq j \leq r} \operatorname{dim}\left(H_{1 j}\right) m_{j}^{\prime}\right)-n_{1}^{\prime}, \\
\mathbf{n}_{l}=\operatorname{dim}\left(\mathbb{N}_{l}\right) \quad \text { si } 1 \leq l \leq r+s-p-1 .
\end{gathered}
$$

Remarquons que $\mathbb{M}_{1}, \ldots, \mathbb{M}_{p}, \mathbb{N}_{1}, \ldots, \mathbb{N}_{r+s-p-1}$ sont des sous-espaces vectoriels de $M_{1}^{(p)}, \ldots, M_{p}^{(p)}, N_{1}^{(p)}, \ldots, N_{r+s-p-1}^{(p)}$ respectivement. On définit une suite $\left(\alpha_{1}^{\prime}, \ldots, \alpha_{p+1}^{\prime}, \beta_{1}^{\prime}, \ldots, \beta_{r+s-p-1}^{\prime}\right)$ de nombres rationnels par les identités

$$
\sum_{1 \leq i \leq r} \lambda_{i} m_{i}^{\prime}-\sum_{1 \leq l \leq s} \mu_{l} n_{l}^{\prime}=\sum_{1 \leq i \leq p+1} \alpha_{i}^{\prime} \mathbf{m}_{i}-\sum_{1 \leq l \leq r+s-p-1} \beta_{l}^{\prime} \mathbf{n}_{l} .
$$

On a donc

$$
\begin{gathered}
\alpha_{i}^{\prime}=\lambda_{i} \quad \text { si } 1 \leq i \leq p, \quad \alpha_{p+1}^{\prime}=\mu_{1}, \\
\beta_{l}^{\prime}=\mu_{1} \operatorname{dim}\left(H_{1, l+p}\right)-\lambda_{l+p} \quad \text { si } 1 \leq l \leq r-p, \\
\beta_{l}^{\prime}=\mu_{l+r-p+1} \quad \text { si } r-p+1 \leq l \leq r+s-p-1 .
\end{gathered}
$$

On normalise ensuite, pour obtenir la suite $\left(\alpha_{1}, \ldots, \alpha_{p+1}, \beta_{1}, \ldots, \beta_{r+s-p-1}\right)$ vérifiant

$$
\sum_{1 \leq i \leq p+1} \alpha_{i} \operatorname{dim}\left(M_{i}^{(p)}\right)=\sum_{1 \leq l \leq r+s-p-1} \beta_{l} \operatorname{dim}\left(N_{l}^{(p)}\right)=1 .
$$

On a donc

avec

$$
\alpha_{i}=\frac{\alpha_{i}^{\prime}}{c}, \quad \beta_{l}=\frac{\beta_{l}^{\prime}}{c},
$$

$$
c=\sum_{1 \leq i \leq p} \lambda_{i} m_{i}+\mu_{1}\left(\left(\sum_{p+1 \leq j \leq r} m_{j} \operatorname{dim}\left(H_{1 j}\right)\right)-n_{1}\right) .
$$

Définition 9. On appelle $\left(\alpha_{1}, \ldots, \alpha_{p+1}, \beta_{1}, \ldots, \beta_{r+s-p-1}\right)$ la polarisation associée $\grave{a}$ $\left(\lambda_{1}, \ldots, \lambda_{r}, \mu_{1}, \ldots, \mu_{s}\right)$. C'est une polarisation de l'action de $G(p)$ sur $W^{\prime}(p)$.

Hypothèse : Dans toute la suite on supposera que les $\alpha_{i}$ et les $\beta_{l}$ sont positifs.

\subsection{Comparaison des (semi-)stabilités}

On veut comparer la (semi-)stabilité d'un élément de $W_{p}^{0}$ avec celle des éléments de $W_{0}^{\prime}(p)$ associés. 
Proposition 5.2. On suppose que

$$
\sum_{1 \leq i \leq p} \lambda_{i} m_{i} \leq \mu_{1}
$$

Si $w \in W_{p}^{0}$ n'est pas $G$-(semi-)stable relativement $\grave{a}\left(\lambda_{1}, \ldots, \lambda_{r}, \mu_{1}, \ldots, \mu_{s}\right)$, alors $z(w) \in W_{0}^{\prime}(p)$ n'est pas $G(p)$-(semi-) stable relativement $\grave{a}\left(\alpha_{1}, \ldots, \alpha_{p+1}, \beta_{1}, \ldots, \beta_{r+s-p-1}\right)$.

Démonstration. On ne traitera que le cas de la semi-stabilité, la stabilité étant analogue. Posons $w=\left(\psi_{l i}\right)_{1 \leq i \leq r, 1 \leq l \leq s}$. Soient $M_{i}^{\prime} \subset M_{i}, N_{l}^{\prime} \subset N_{l}$ des sous-espaces vectoriels tels que

$$
\epsilon=\sum_{1 \leq i \leq r} \lambda_{i} \operatorname{dim}\left(M_{i}^{\prime}\right)-\sum_{1 \leq l \leq s} \mu_{l} \operatorname{dim}\left(N_{l}^{\prime}\right)>0
$$

et $\psi_{l i}\left(H_{l i}^{*} \otimes M_{i}^{\prime}\right) \subset N_{l}^{\prime}$ pour $1 \leq i \leq r, 1 \leq l \leq s$. On peut supposer que

$$
N_{1}^{\prime}=\sum_{p+1 \leq j \leq r} \psi_{1 j}\left(H_{1 j}^{*} \otimes M_{j}^{\prime}\right) .
$$

En effet, supposons que

$$
k=\operatorname{codim}_{N_{1}^{\prime}}\left(\sum_{p+1 \leq j \leq r} \psi_{1 j}\left(H_{1 j}^{*} \otimes M_{j}^{\prime}\right)\right)>0 .
$$

On a, en posant $m_{i}^{\prime}=\operatorname{dim}\left(M_{i}^{\prime}\right), n_{l}^{\prime}=\operatorname{dim}\left(N_{l}^{\prime}\right)$,

$$
\begin{aligned}
\sum_{p+1 \leq i \leq r} \lambda_{i} m_{i}^{\prime}-\mu_{1}\left(n_{1}^{\prime}-k\right) & =\epsilon-\sum_{1 \leq i \leq p} \lambda_{i} m_{i}^{\prime}+k \mu_{1}+\sum_{2 \leq l \leq s} \mu_{l} n_{l}^{\prime} \\
& >k \mu_{1}-\sum_{1 \leq i \leq p} \lambda_{i} m_{i}^{\prime}>0
\end{aligned}
$$

par hypothèse. On peut donc au besoin remplacer $\left(M_{1}^{\prime}, \ldots, M_{r}^{\prime}, N_{1}^{\prime}, \ldots, N_{s}^{\prime}\right)$ par

$$
\left(0, \ldots, 0, M_{p+1}^{\prime}, \ldots, M_{r}^{\prime}, \sum_{p+1 \leq j \leq r} \psi_{1 j}\left(H_{1 j}^{*} \otimes M_{j}^{\prime}\right), 0, \ldots, 0\right) .
$$

Soient $\mathbb{M}_{i}=M_{i}^{\prime} \subset M_{i}^{(p)}$ pour $1 \leq i \leq p$,

$$
\begin{gathered}
\mathbb{M}_{p+1}=\operatorname{ker}\left(\sum_{p+1 \leq j \leq r} \psi_{1 j}\right) \cap\left(\bigoplus_{p+1 \leq j \leq r}\left(H_{1 j}^{*} \otimes M_{j}^{\prime}\right)\right) \subset M_{p+1}^{(p)}=\operatorname{ker}\left(\sum_{p+1 \leq j \leq r} \psi_{1 j}\right), \\
\mathbb{N}_{l}=M_{l+p}^{\prime} \subset N_{l}^{(p)} \text { si } 1 \leq l \leq r-p, \quad \mathbb{N}_{l}=N_{l-r+p+1}^{\prime} \quad \text { si } r-p+1 \leq l \leq r+s-p-1 .
\end{gathered}
$$

Il faut s'arranger pour trouver

$$
z(w)=\left(\begin{array}{ll}
\phi_{11}^{\prime} & \phi_{12}^{\prime} \\
h_{21}^{\prime} & h_{22}^{\prime}
\end{array}\right)=\left(\psi_{l i}^{\prime}\right)_{1 \leq i \leq p+1,1 \leq l \leq r+s-p-1}
$$

de telle sorte que

$$
\psi_{l i}^{\prime}\left(H_{l i}^{(p) *} \otimes \mathbb{M}_{i}\right) \subset \mathbb{N}_{l} \text { pour } 1 \leq i \leq p+1,1 \leq l \leq r+s-p-1 .
$$

On peut prendre $u$ tel que

$$
u\left(\bigoplus_{p+1 \leq j \leq r, 2 \leq l \leq s}\left(H_{1 j}^{*} \otimes B_{l 1}^{*} \otimes M_{j}^{\prime}\right)\right) \subset \bigoplus_{2 \leq l \leq s} N_{l}^{\prime},
$$


et $v$ tel que

$$
v\left(\bigoplus_{1 \leq i \leq p}\left(H_{1 i}^{*} \otimes M_{i}^{\prime}\right)\right) \subset \bigoplus_{p+1 \leq j \leq r}\left(H_{1 j}^{*} \otimes M_{j}^{\prime}\right)
$$

$\left(\operatorname{car} \quad N_{1}^{\prime}=\overline{\phi_{2}}\left(\bigoplus_{p+1 \leq j \leq r}\left(H_{1 j}^{*} \otimes M_{j}^{\prime}\right)\right)\right)$. Dans ce cas $z(w)$ possède les propriétés voulues.

Corollaire 5.3. Si

$$
\mu_{1} \geq \frac{1}{n_{1}+1}
$$

et si $w \in W_{p}^{0}$ est tel que $z(w)$ n'est pas $G(p)$-(semi-) stable relativement à $\left(\alpha_{1}, \ldots, \alpha_{p+1}, \beta_{1}, \ldots, \beta_{r+s-p-1}\right)$, alors $w$ n'est pas $G$-(semi-)stable relativement $\grave{a}$ $\left(\lambda_{1}, \ldots, \lambda_{r}, \mu_{1}, \ldots, \mu_{s}\right)$

Démonstration. . On applique la proposition précédente à la mutation inverse.

Le résultat suivant est immédiat :

Proposition 5.4. Si

alors on a $W^{s s} \subset W_{p}^{0}$.

$$
\mu_{1<} \frac{\sum_{p+1 \leq j \leq r} \lambda_{j} m_{j}}{n_{1}-1},
$$

Corollaire 5.5. Si

$$
\mu_{1}>\frac{\sum_{p+1 \leq j \leq r} \lambda_{j} m_{j}}{n_{1}+1},
$$

alors on a $W^{\prime}(p)^{s s} \subset W_{0}^{\prime}(p)$.

\subsection{Construction de quotients}

\subsubsection{Quasi-bons quotients}

On déduit de ce qui précède et du théorème 4.12 le

Théorème 5.6. Si

$$
\operatorname{Max}\left(\frac{1}{n_{1}+1}, 1-\sum_{p+1 \leq j \leq r} \lambda_{j} m_{j}\right) \leq \mu_{1}<\frac{\sum_{p+1 \leq j \leq r} \lambda_{j} m_{j}}{n_{1}-1},
$$

alors il existe un quasi-bon quotient $W^{\prime}(p)^{s s} / / G(p)$ si et seulement si il existe un quasi-bon quotient $W^{s s} / / G$, et dans ce cas les deux quotients sont isomorphes. 
Cas particuliers :

1 - Si $p=0$, la condition du théorème précédent se réduit à

$$
\mu_{1} \geq \frac{1}{n_{1}+1}
$$

2 - Si $s=1$, la condition du théorème précédent se réduit à

$$
\sum_{p+1 \leq j \leq r} \lambda_{j} m_{j} \geq \frac{n_{1}-1}{n_{1}} .
$$

3 - Si $p=0$ et $s=1$, la condition du théorème précédent est toujours vérifiée.

\subsubsection{Bons quotients}

On déduit du $\$ 5.6$ de la proposition 4.9 le

Théorème 5.7. On suppose que les propriétés suivantes sont vérifiées :

(1) On a $\operatorname{Max}\left(\frac{1}{n_{1}+1}, 1-\sum_{p+1 \leq j \leq r} \lambda_{j} m_{j}\right) \leq \mu_{1}<\frac{\sum_{p+1 \leq j \leq r} \lambda_{j} m_{j}}{n_{1}-1}$.

(2) Il existe un bon quotient $W^{s s} / / G$.

(3) Il existe un caractère $\chi$ de $G$ tel que $W^{s s}=W^{s s}(\chi)$.

(4) On a $\operatorname{codim}\left(W \backslash W^{s s}\right) \geq 2$.

(5) Il existe un recouvrement de $W^{s s} / / G$ par des ouverts affines dont l'image inverse dans $W^{\text {ss }}$ soit de la forme $W_{f}$ (où $f$ est un polynôme $\chi^{k}$-invariant, pour un entier $k>0$ ).

Alors il existe un bon quotient $W^{\prime}(p)^{s s} / / G(p)$ qui est isomorphe ̀̀ $W^{s s} / / G$.

Remarquons que les propriétés 3 et 5 sont vérifiées si $W^{s s} / / G$ est un des bons quotients construits dans [6]. Enfin, on déduit de la proposition 4.10 le

Théorème 5.8. On suppose que $p=0, \mu_{1} \geq \frac{1}{n_{1}+1}$, et qu'il existe un bon quotient $W^{s s} / / G$. Alors il existe un bon quotient $W^{\prime}(p)^{s s} / / G(p)$ qui est isomorphe ̀̀ $W^{s s} / / G$.

Démonstration. Dans ce cas les mutations de morphismes de type $(r, s)$ sont de type $(1, r+s-1)$. Il suffit de prouver que toute hypersurface $G$-invariante de $W$ contient le complémentaire de $W_{p}^{0}$, et que toute hypersurface $G(p)$-invariante de $W^{\prime}(p)$ contient le complémentaire de $W_{0}^{\prime}(p)$. Rappelons que dans ce cas $W_{p}^{0}$ est l'ouvert de $W$ constitué des morphismes $\bigoplus_{1 \leq i \leq r}\left(\mathcal{E}_{i} \otimes M_{i}\right) \longrightarrow \bigoplus_{1 \leq j \leq s}\left(\mathcal{F}_{j} \otimes N_{j}\right)$ tels que l'application linéaire induite $\underset{1 \leq i \leq r}{\bigoplus} H_{1 i}^{*} \otimes M_{i} \longrightarrow N_{1}$ soit surjective. Il est immédiat que pour tout choix de la polarisation, les points de $W \backslash W_{p}^{0}$ sont non semi-stables. Soit $Z_{0}$ une hypersurface $G$-invariante de $W$. Alors son équation $f$ est un 
polynôme $\chi_{0}$-invariant, $\chi_{0}$ étant un caractère non trivial de $G$ (cela découle du fait que toute fonction régulière inversible sur $G$ est le produit d'un caractère de $G$ par une constante et de la proposition 14 de [2]). A ce caractère correspond une polarisation $\Lambda^{\prime}$ de l'action de $G$ (cf. [6] et [9]) telle que les points de $W \backslash Z_{0}$ sont $G_{r e d}$-semi-stables relativement à $\Lambda^{\prime}$. On a donc $W \backslash W_{p}^{0} \subset Z_{0}$. On montre de même que toute hypersurface $G(p)$-invariante de $W^{\prime}(p)$ contient le complémentaire de $W_{0}^{\prime}(p)$.

5.7.3. Variétés de modules de morphismes de type $(2,1)$

On suppose maintenant que $r=2$ et $s=1$, c'est-à-dire qu'on veut construire des variétés de modules de morphismes du type

$$
\left(\mathcal{E}_{1} \otimes M_{1}\right) \oplus\left(\mathcal{E}_{2} \otimes M_{2}\right) \longrightarrow \mathcal{F}_{1} \otimes N_{1} .
$$

On utilise les notations du $\$ 5.4 .2$, Soient

$$
\tau^{*}: H_{12} \otimes A_{21} \longrightarrow H_{11}
$$

la composition, et

$$
\tau: H_{11}^{*} \otimes A_{21} \longrightarrow H_{12}^{*}
$$

l'application déduite de $\tau^{*}$. On déduit de ce qui précède l'amélioration suivante du théorème 5.1:

Théorème 5.9. Si $r=2$ et $s=1$, il existe un bon quotient projectif $W^{s s} / / G$ dans chacun des deux cas suivants:

1 - On a

$$
t>\frac{m_{2} \operatorname{dim}\left(\operatorname{Hom}\left(\mathcal{E}_{1}, \mathcal{E}_{2}\right)\right)}{\operatorname{dim}\left(\operatorname{Hom}\left(\mathcal{E}_{1}, \mathcal{E}_{2}\right)\right)+m_{1}} \quad \text { et } \quad t>\operatorname{dim}\left(\operatorname{Hom}\left(\mathcal{E}_{1}, \mathcal{E}_{2}\right)\right) \cdot c\left(\tau, m_{2}\right) \frac{m_{2}}{n_{1}}
$$

2 - On a, en posant $b=\operatorname{dim}\left(\operatorname{Hom}\left(\mathcal{E}_{1}, \mathcal{E}_{2}\right)\right) \operatorname{dim}\left(\operatorname{Hom}\left(\mathcal{E}_{2}, \mathcal{F}_{1}\right)\right)-\operatorname{dim}\left(\operatorname{Hom}\left(\mathcal{E}_{1}, \mathcal{F}_{1}\right)\right)$,

$$
t<\frac{m_{2}}{n_{1}} \operatorname{dim}\left(\operatorname{Hom}\left(\mathcal{E}_{2}, \mathcal{F}_{1}\right)\right), \quad t>\frac{m_{2}}{n_{1}} \cdot \frac{b m_{1}+n_{1}}{\operatorname{dim}\left(\operatorname{Hom}\left(\mathcal{E}_{1}, \mathcal{E}_{2}\right)\right) m_{1}+m_{2}},
$$

et

$$
t>1-\frac{m_{1}}{n_{1}}\left(\operatorname{dim}\left(\operatorname{Hom}\left(\mathcal{E}_{1}, \mathcal{F}_{1}\right)\right)-\operatorname{dim}\left(\operatorname{Hom}\left(\mathcal{E}_{1}, \mathcal{E}_{2}\right)\right) c\left(\tau^{*}, m_{1}\right)\right)
$$

Démonstration. Les cas 1 sont ceux du théorème 5.1. Pour obtenir les cas 2, on emploie le théorème 5.8 et on applique le théorème 5.1 à l'espace $W^{\prime}(0)$ des mutations de morphismes. 


\section{Applications}

\subsection{Calcul de constantes}

Soient $E, H, F$ des espaces vectoriels de dimension finie et

$$
\tau: E \otimes H \longrightarrow F
$$

une application linéaire. Soit $M$ un espace vectoriel de dimension $m>0$. On note

$$
\tau_{m}=\tau \otimes I_{M}: E \otimes(H \otimes M) \longrightarrow F \otimes M .
$$

On dit qu'un sous-espace vectoriel $K$ de $H \otimes M$ est générique s'il est propre et si pour tout sous-espace vectoriel propre $M^{\prime}$ de $M, K$ n'est pas contenu dans $H \otimes M^{\prime}$. On note $\mathcal{K}$ l'ensemble des sous-espaces vectoriels génériques de $H \otimes M$. Si $K$ est un sous-espace vectoriel générique de $H \otimes M$ on note

$$
\delta(K)=\frac{\operatorname{codim}\left(\tau_{m}(E \otimes K)\right)}{\operatorname{codim}(K)} .
$$

On pose

$$
c_{\tau}(m)=\sup _{K \in \mathcal{K}}(\delta(K))
$$

Nous aurons besoin des constantes correspondant aux applications $\tau$ suivantes:

$$
\begin{gathered}
\sigma_{0}: S^{2} V \otimes V^{*} \longrightarrow V, \\
\sigma_{1}: V \otimes V \longrightarrow S^{2} V,
\end{gathered}
$$

On posera

$$
c_{i}(m)=c_{\sigma_{i}}(m)
$$

pour $i=0,1$. On montrera aux $\$ 6.1 .2$ et 6.1 .3 la

Proposition 6.1. On a

$$
\begin{aligned}
c_{0}(m)=\frac{m(m-1)}{2(m(n+1)-1)} \quad \text { si } \quad m \leq n+1, \quad c_{0}(m)=\frac{n+1}{2(n+2)} \quad \text { si } \quad m \geq n+1, \\
c_{1}(m)=\frac{(n+1)(m(n+2)-2)}{2(m(n+1)-1)} \quad \text { si } \quad m \leq n+1, \quad c_{1}(m)=\frac{(n+1)(n+3)}{2(n+2)} \quad \text { si } \quad m \geq n+1 .
\end{aligned}
$$

\subsubsection{Propriétés générales}

On considère l'application

$$
\tau: E \otimes H \longrightarrow F
$$

et les $\tau_{m}$ dérivées. Soit $u \in H \otimes M$ non nul. On appelle longueur de $u$ le plus petit entier $d$ tel qu'il existe des éléments $h_{1}, \ldots, h_{d}$ de $H, x_{1}, \ldots, x_{d}$ de $M$ tels que

$$
u=h_{1} \otimes x_{1}+\cdots+h_{d} \otimes x_{d} .
$$

C'est aussi la dimension du plus petit sous-espace vectoriel $M^{\prime} \subset M$ tel que $u \in H \otimes M^{\prime}$. 
Proposition 6.2. Soit $K \in \mathcal{K}$ tel qu'il existe un élément non nul de $K$ de longueur $m^{\prime}<m$. Alors on a

$$
\frac{\operatorname{codim}\left(\tau_{m}(E \otimes K)\right)}{\operatorname{codim}(K)} \leq \sup \left(c_{\tau}\left(m^{\prime}\right), c_{\tau}\left(m-m^{\prime}\right)\right)
$$

Démonstration. Soient $u \in K$ un élément de longueur $m^{\prime}$ et $M^{\prime}$ le plus petit sous-espace vectoriel de $M$ tel que $H \otimes M^{\prime}$ contienne $u$. Soient $p: H \otimes M \rightarrow H \otimes\left(M / M^{\prime}\right)$ la projection et

$$
K^{\prime}=K \cap\left(H \otimes M^{\prime}\right), \quad K^{\prime \prime}=p(K) .
$$

Alors $M^{\prime}$ (resp. $M / M^{\prime}$ ) est le plus petit sous-espace vectoriel $M^{\prime \prime}$ de $M^{\prime}$ (resp. $M / M^{\prime}$ ) tel que $K \subset H \otimes M^{\prime \prime}$ (resp. $\left.K^{\prime \prime} \subset H \otimes M^{\prime \prime}\right)$. On a donc

$$
\begin{gathered}
\operatorname{codim}\left(\tau_{m^{\prime}}\left(E \otimes K^{\prime}\right)\right) \leq c_{\tau}\left(m^{\prime}\right) \operatorname{codim}_{H \otimes M^{\prime}}\left(K^{\prime}\right), \\
\operatorname{codim}\left(\tau_{m-m^{\prime}}\left(E \otimes K^{\prime \prime}\right)\right) \leq c_{\tau}\left(m-m^{\prime}\right) \operatorname{codim}_{H \otimes\left(M / M^{\prime}\right)}\left(K^{\prime \prime}\right) .
\end{gathered}
$$

Comme $\operatorname{dim}(K)=\operatorname{dim}\left(K^{\prime}\right)+\operatorname{dim}\left(K^{\prime \prime}\right)$ on obtient le résultat voulu.

Corollaire 6.3. Si $m \geq \operatorname{dim}(H)$ on a $c_{\tau}(m)=c_{\tau}(\operatorname{dim}(H))$.

Démonstration. Cela découle de la proposition précédente et de [6], lemma 7.1.1.

\subsubsection{Calcul de $c_{0}(m)$}

D'après le corollaire 6.3 il suffit de montrer que

$$
c_{0}(m)=\frac{m(m-1)}{2(m(n+1)-1)}
$$

si $1 \leq m \leq n+1$. Soit $m=\operatorname{dim}(M)$, avec $1 \leq m \leq n+1$. On vérifie aisément que la formule précédente est la valeur de $\delta(K)$ lorsque $K$ est de dimension 1 et engendré par un élément de longueur $m$. D'après la proposition 6.2 il suffit de montrer que si tous les éléments non nuls de $K$ sont de longueur $m$ et si $d=\operatorname{dim}(K)>1$, alors on a $\tau_{m}\left(S^{2} V \otimes K\right)=V \otimes M$. Il suffit de considérer le cas $d=2$. Soient $\left(x_{1}, \ldots, x_{m}\right)$ une base de $M$ et

$$
\left(z_{1} \otimes x_{1}+\cdots, z_{m} \otimes x_{m}, z_{1}^{\prime} \otimes x_{1}, \cdots, z_{m}^{\prime} \otimes x_{m}\right)
$$

une base de $K$. Alors

$$
\operatorname{dim}\left(<z_{1}, \ldots, z_{m}, z_{1}^{\prime}, \ldots, z_{m}^{\prime}>\right)>m
$$

car dans le cas contraire $K$ contiendrait des éléments de longueur inférieure à $m$. On peut donc supposer que $z_{m}^{\prime}$ n'appartient pas au sous-espace vectoriel de $V$ engendré par $z_{1}, \ldots, z_{m}, z_{1}^{\prime}, \ldots, z_{m-1}^{\prime}$. On procède maintenant par récurrence sur $m$. Pour $m=1$, on a déjà $\tau\left(S^{2} V \otimes<z_{1}>\right)=V$, donc a fortiori $\tau\left(S^{2} V \otimes K\right)=V$. Supposons donc que le résultat soit vrai pour $m-1$. Soient $W=z_{m}^{\prime}{ }^{\perp}$ et $e_{m}^{\prime} \in V$ tel que $z_{m}^{\prime}\left(e_{m}^{\prime}\right)=1$ et orthogonal à $z_{1}, \ldots, z_{m}, z_{1}^{\prime}, \ldots, z_{m-1}^{\prime}$. On identifie $W^{*}$ à $e_{m}^{\prime}{ }^{\perp}$. Soit $M^{\prime}=<x_{1}, \ldots, x_{m-1}>$. Soit $K^{\prime}$ le sousespace vectoriel de $W^{*} \otimes M^{\prime}$ engendré par

$$
x_{1} \otimes z_{1}+\cdots+x_{m-1} \otimes z_{m-1}, \quad x_{1} \otimes z_{1}^{\prime}+\cdots+x_{m-1} \otimes z_{m-1}^{\prime} .
$$


Soient maintenant $v_{i}=v_{i}^{\prime}+\alpha_{i} e_{m}^{\prime} \in V, 1 \leq i \leq m$, avec $v_{i}^{\prime} \in W^{\prime}$. On peut d'après l'hypothèse de récurrence trouver $q_{0}, q_{0}^{\prime} \in S^{2} W$ tels que

$$
q_{0}\left(z_{i}\right)+q_{0}^{\prime}\left(z_{i}^{\prime}\right)=v_{i}^{\prime}
$$

pour $1 \leq i \leq m-1$. Soient $u, u^{\prime} \in V$ et

$$
q=q_{0}+u e_{m}^{\prime}, \quad q^{\prime}=q_{0}^{\prime}+u^{\prime} e_{m}^{\prime} .
$$

On veut obtenir $q\left(z_{i}\right)+q^{\prime}\left(z_{i}^{\prime}\right)=v_{i}$ pour $1 \leq i \leq m$. Ces équations équivalent à

$$
\begin{gathered}
z_{i}(u)+z_{i}^{\prime}\left(u^{\prime}\right)=\alpha_{i} \text { pour } 1 \leq i \leq m-1, \\
u^{\prime}+q_{0}\left(z_{m}\right)+\left(z_{m}(u)+z_{m}^{\prime}\left(u^{\prime}\right)\right) e_{m}^{\prime}=v_{m} .
\end{gathered}
$$

La seconde équation permet (en fixant $z_{m}(u)=0$ ) de déterminer $u^{\prime}$ et les autres permettent alors de déterminer $u$. On a donc bien prouvé que $\tau\left(S^{2} V \otimes K\right)=V \otimes M$.

\subsubsection{Calcul de $c_{1}(m)$}

D'après le corollaire 6.3 il suffit de montrer que

$$
c_{1}(m)=\frac{(n+1)(m(n+2)-2)}{2(m(n+1)-1)}
$$

si $1 \leq m \leq n+1$. Soit $m=\operatorname{dim}(M)$, avec $1 \leq m \leq n+1$. On vérifie aisément que la formule précédente est la valeur de $\delta(K)$ lorsque $K$ est de dimension 1 et engendré par un élément de longueur $m$. D'après la proposition 6.2 il suffit de montrer que si tous les éléments non nuls de $K$ sont de longueur $m$ et si $d=\operatorname{dim}(K)>1$, alors on a

$$
\delta(K)<\frac{(n+1)(m(n+2)-2)}{2(m(n+1)-1)} .
$$

Supposons d'abord que $m=1$. Alors on a $K \subset V, \tau_{1}(V \otimes K)=V . K \subset S^{2} V$. Donc $\operatorname{codim}\left(\tau_{1}(V \otimes K)\right)=\operatorname{dim}\left(S^{2}(V / K)\right)$ et

$$
\delta(K)=\frac{\operatorname{dim}(V / K)}{2}<\frac{n+1}{2} .
$$

On suppose maintenant que $m>1$. On va montrer que la restriction de $\tau: V \otimes K \rightarrow S^{2} V \otimes M$ est injective. Supposons le contraire. Soient $u_{1}, \ldots, u_{p}$ des éléments de $K$ linéairement indépendants et $v_{1}, \ldots, v_{p} \in V$ non tous nuls tels que

$\tau_{m}\left(v_{1} \otimes u_{1}+\cdots v_{p} \otimes u_{p}\right)=0$. On suppose que $p$ est minimal, ce qui entraîne que $v_{1}, \ldots, v_{p}$ sont linéairement indépendants. Soit $V^{\prime} \subset V$ le sous-espace vectoriel engendré par $v_{1}, \ldots, v_{p}$. Posons pour $1 \leq i \leq p$

$$
u_{i}=w_{1}^{i} \otimes x_{1}+\cdots w_{m}^{i} \otimes x_{m}
$$

avec $w_{j}^{i} \in V,\left(x_{1}, \ldots, x_{m}\right)$ étant une base de $M$. On a alors pour $1 \leq j \leq m$

$$
v_{1} w_{j}^{1}+\ldots v_{p} w_{j}^{p}=0 .
$$

Ceci entraîne que $w_{j}^{i} \in V^{\prime}$. Il existe donc une combinaison linéaire non nulle de $\left(w_{1}^{1}, \ldots, w_{1}^{p}\right)$ et $\left(w_{2}^{1}, \ldots, w_{2}^{p}\right)$ qui n'est pas contituée de vecteurs linéairement indépendants. En changeant la base $\left(x_{1}, \ldots, x_{m}\right)$ de $M$ on peut donc se ramener au cas où $w_{1}^{1}, \ldots, w_{1}^{p}$ ne sont pas linéairement indépendants. Comme $u_{1}, \ldots, u_{p}$ le sont, ceci entraîne qu'on peut trouver dans $K$ un 
élément non nul de longueur strictement inférieure à $m$. Ceci est contraire à l'hypothèse. Donc la restriction de $\tau$ à $V \otimes K$ est injective. On a donc, en posant $d=\operatorname{dim}(K)$,

$$
\operatorname{dim}\left(\tau_{m}(V \otimes K)\right)=(n+1) d,
$$

et on vérifie aisément que

$$
\delta(K)<\frac{(n+1)(m(n+2)-2)}{2(m(n+1)-1)} .
$$

Ceci achève la démonstration de la proposition 6.1 .

\subsection{Variétés de modules de morphismes $m_{1} \mathcal{O}(-2) \oplus m_{2} \mathcal{O}(-1) \longrightarrow n_{1} \mathcal{O}$ sur $\mathbb{P}_{n}$}

On applique le théorème 5.9 aux morphismes

$$
\left(\mathcal{O}(-2) \otimes \mathbb{C}^{m_{1}}\right) \oplus\left(\mathcal{O}(-1) \otimes \mathbb{C}^{m_{2}}\right) \longrightarrow \mathcal{O} \otimes \mathbb{C}^{n_{1}}
$$

compte tenu des calculs du $\S 6.1$. On pose

$$
\eta_{1}=\frac{m_{1}}{n_{1}}, \quad \eta_{2}=\frac{m_{2}}{n_{1}} .
$$

On obtient le résultat suivant, qui généralise celui du $§ 10.2$ de [6] :

Théorème 6.4. Il existe un bon quotient projectif $W^{s s} / / G$ dans chacun des deux cas suivants :

$1-$ On $a$

$$
\begin{aligned}
& t> \frac{(n+1) \eta_{2}}{(n+1) \eta_{2}+\eta_{1}}, \\
& t> \frac{(n+1) m_{2}\left(m_{2}-1\right)}{2\left(m_{2}(n+1)-1\right)} \eta_{2} \text { si } 2 \leq m_{2} \leq n+1 \\
& t>\frac{(n+1)^{2}}{2(n+2)} \eta_{2} \text { si } m_{2}>n+1 .
\end{aligned}
$$

2-On a

$$
\begin{aligned}
& t<(n+1) \eta_{2} \\
& t> \frac{n(n+1)}{(n+1) \frac{\eta_{1}}{\eta_{2}}+1} \eta_{1}+1 \\
& t>1-\frac{n(n+1)}{2\left(m_{1}(n+1)-1\right)} \eta_{1} \text { si } m_{1} \leq n+1, \\
& t>1-\frac{n+1}{2(n+2)} \eta_{1} \text { si } m_{1}>n+1 .
\end{aligned}
$$

La partie 1- s'obtient directement à partir de [6] et des calculs de constantes du $\S 6.1$, et la partie 2- en utilisant des mutations des morphismes. 


\subsection{Exemple 1}

On considère les morphismes

$$
\left(\phi_{1}, \phi_{2}\right): \mathcal{O}(-2) \oplus \mathcal{O}(-1) \longrightarrow \mathcal{O} \otimes \mathbb{C}^{n+2}
$$

$\operatorname{sur} \mathbb{P}_{n}$.

On sait d'après les résultats de [6] (résumés dans le théorème 6.4, 1-) construire un bon quotient $W^{s s} / / G$ dés que

$$
t=\lambda_{2}>\frac{n+1}{n+2}
$$

Mais dans ce cas le quotient est vide! En effet, il existe toujours un sous-espace vectoriel $H \subset \mathbb{C}^{n+2}$ de dimension $n+1$ tel que $\operatorname{Im}\left(\phi_{2}\right) \subset \mathcal{O} \otimes H$. On doit donc avoir, si $\left(\phi_{1}, \phi_{2}\right)$ est $G$-semi-stable relativement à $\left(\lambda_{1}, \lambda_{2}, \mu_{1}\right), \lambda_{2}-\frac{n+1}{n+2} \leq 0$.

On emploie maintenant le théorème 6.4. On peut construire un bon quotient $W^{s s} / / G$ dés que

$$
t>\frac{n+3}{2(n+2)}
$$

Les valeurs singulières de $t$ sont par définition celles pour lesquelles la $G$-semi-stabilité n'implique pas la $G$-stabilité. Ces valeurs sont exactement les nombres

$$
t_{k}=\frac{k}{n+2}
$$

pour $1 \leq k \leq n+1$. Dans ce cas un morphisme $\left(\phi_{1}, \phi_{2}\right) G$-semi-stable non $G$-stable est construit de la façon suivante : on considère un sous-espace vectoriel $H \subset \mathbb{C}^{n+2}$ de dimension $k$, et on prend pour $\phi_{2}$ un morphisme tel que $\operatorname{Im}\left(\phi_{2}\right) \subset \mathcal{O} \otimes H$ et que $H$ soit le plus petit sousespace vectoriel ayant cette propriété. On prend pour $\phi_{1}$ un morphisme tel que l'application linéaire induite

$$
H^{0}(\mathcal{O}(2))^{*} \longrightarrow \mathbb{C}^{n+2}
$$

soit surjective.

On obtient donc au total $n$ quotients distincts et non vides, dont $\left[\frac{n+1}{2}\right]$ sont singuliers. Ils sont de dimension $\frac{(n+2)\left(n^{2}+3 n-2\right)}{2}$, sauf celui correspondant à $t_{n+1}$, qui est de dimension $\frac{n(n+3)}{2}$.

On peut généraliser ce qui précède et obtenir des bons quotients projectifs d'espaces de morphismes du type

$$
\mathcal{O}(-p-q) \oplus \mathcal{O}(-p) \longrightarrow \mathcal{O} \otimes \mathbb{C}^{n+2}
$$

sur $\mathbb{P}_{n}, p, q$ étant des entiers positifs. 


\subsection{Exemple 2}

On considère les morphismes

$$
\mathcal{O}(-2) \oplus\left(\mathcal{O}(-1) \otimes \mathbb{C}^{k}\right) \longrightarrow \mathcal{O} \otimes \mathbb{C}^{n k+1}
$$

$\operatorname{sur} \mathbb{P}_{n}$.

On sait d'après les résultats de [6] (résumés dans le théorème 6.4 1-) construire un bon quotient $W^{s s} / / G$ dés que

$$
t>1-\frac{1}{1+(n+1) k}=t_{1} .
$$

Si on utilise le théorème 6.4, 2-, on peut construire un bon quotient $W^{s s} / / G$ dés que

$$
t>1-\frac{n+1}{2(n k+1)}=t_{2}
$$

On s'intéresse maintenant aux valeurs singulières de $t$, c'est-à-dire à celles pour lesquelles il peut exister des morphismes semi-stables non stables. Si $\left(\lambda_{1}, \lambda_{2}, 1 /(n k+1)\right)$ est la polarisation correspondant à $t$, ce dernier est singulier si et seulement si il existe des entiers $k^{\prime}, p$, avec $1 \leq k^{\prime}<k, 0 \leq p<n k$, tels que

On a alors

$$
\lambda_{2} k^{\prime}=\frac{n k-p}{n k+1}
$$

$$
t=\frac{k(n k-p)}{k^{\prime}(n k+1)}
$$

Les valeurs singulières de $t$ sont donc les nombres de la forme $\frac{k(n k-p)}{k^{\prime}(n k+1)}$ (où $1 \leq k^{\prime}<k$, $0 \leq p<n k)$ compris strictement entre 0 et 1 .

Lemme 6.5. La plus grande valeur singulière de $t$ est $t_{\max }=\frac{n k}{n k+1}$.

Démonstration. On peut obtenir $t_{\max }$ en prenant $p=n\left(k-k^{\prime}\right)$, donc c'est bien une valeur singulière de $t$. Il reste à montrer que c'est la plus grande. Pour cela il suffit de prouver que si $t=\frac{k(n k-p)}{k^{\prime}(n k+1)}\left(\right.$ avec $\left.1 \leq k^{\prime}<k, 0 \leq p<n k\right)$, et si $0<t<1$, alors on a $1-t \geq \frac{1}{n k+1}$. L'inégalité $t<1$ équivaut à $p>n\left(k-k^{\prime}\right)-\frac{k^{\prime}}{k}$. Ceci entraîne que $p \geq n\left(k-k^{\prime}\right)$, et cette dernière inégalité équivaut à $1-t \geq \frac{1}{n k+1}$.

On vérifie aisément qu'on a

$$
t_{2}<t_{\max }<t_{1}
$$

La variété de modules $M_{+}=W^{s s} / / G$ obtenue pour $t>t_{\max }$ est décrite dans le $\S 10.2$ de [6] : c'est un fibré en grassmanniennes sur la variété $N(n+1, k, n k+1)$ (cf. chapitre 2). C'est la seule que l'on puisse construire en utilisant directement les résultats de [6]. En utilisant les mutations de morphismes on peut donc obtenir les deux variétés de modules supplémentaires suivantes : $M_{0}$ (pour $\left.t=t_{\max }\right)$ et $M_{-}$(pour $t<t_{\max }$ proche de $\left.t_{\max }\right)$. Il est possible de décrire 
complètement les variétés $M_{0}$ et $M_{-}$, ainsi que le passage de $M_{+}$à $M_{-}$en termes de flips, comme dans l'exemple du $\S 10.1$ de [6].

\section{RÉFÉRENCES}

[1] Drézet, J.-M. Fibrés exceptionnels et suite spectrale de Beilinson généralisée sur $\mathbb{P}_{2}(\mathbb{C})$. Math. Ann. 275 (1986), 25-48.

[2] Drézet, J.-M. Fibrés exceptionnels et variétés de modules de faisceaux semi-stables sur $\mathbb{P}_{2}(\mathbb{C})$. Journ. Reine Angew. Math. 380 (1987), 14-58.

[3] Drézet, J.-M. Variétés de modules extrémales de faisceaux semi-stables sur $\mathbb{P}_{2}(\mathbb{C})$.Math. Ann. 290 (1991), 727-770.

[4] Drézet, J.-M. Quotients algébriques par des groupes non réductifs et variétés de modules de complexes. Intern. Journ. of Math. Vol. 9 No. 7 (1998), 769-819.

[5] Drézet, J.-M., Le Potier, J. Fibrés stables et fibrés exceptionnels sur $\mathbb{P}_{2}$. Ann. Ec. Norm. Sup. 18 (1985), 193-244.

[6] Drézet, J.-M., Trautmann, G. Moduli spaces of decomposable morphisms of sheaves and quotients by nonreductive groups. Preprint (1998), e-print service at http ://xxx.lanl.gov/list/math/9805.

[7] Gorodentsev, A.L., Rudakov, A.N. Exceptional vector bundles on projective spaces . Duke Math. Journ. 54 (1987), 115-130.

[8] Karpov B.V. Semi-stable sheaves on a two-dimensional quadric and Kronecker modules. Math. Izvestiya AMS transl. 40 (1993), 33-66.

[9] King, A. Moduli of representations of finite dimensional algebras . Quart. Journ. of Math. Oxford 45 (1994),515-530.

[10] Miró-Roig, R.M. Some moduli spaces for rank 2 stable reflexive sheaves on $\mathbb{P}_{3}$. Trans. Amer. Math. Soc. 299 (1987), 699-717.

[11] Miró-Roig, R.M., Trautmann, G.The moduli scheme $M(0,2,4)$ over $\mathbb{P}_{3}$. Math. Z. 216 (1994), 283-315.

[12] Mumford, D., Fogarty, J. Geometric invariant theory. Ergeb. Math. Grenzgeb. Bd. 34. Berlin Heidelberg New-York : Springer (1982)

[13] Newstead, P.E. Introduction to moduli problems and orbit spaces. TIFR Lect. Notes. Math. vol. 51. Berlin Heidelberg New-York : Springer (1978)

[14] Okonek, C. Moduli extremer reflexiver Garben auf $\mathbb{P}^{n}$. Journ. Reine Angew. Math. 338 (1983), 183-194.

Notes : Ce texte reproduit l'article

Espaces abstraits de morphismes et mutations. Journal für die reine und angewandte Mathematik 518 (2000) , 41-93.

avec quelques améliorations dans la rédaction et la bibliographie. En particulier une erreur dans la formule du théorème 5.1 a été corrigée. 
Institut de Mathématiques de Jussieu, Case 247, 4 Place Jussieu, F-75252 Paris, France

$U R L:$ http://www.math.jussieu.fr/ drezet 